\title{
CONODONT AGE AND CAI DATA FOR NEW JERSEY
}

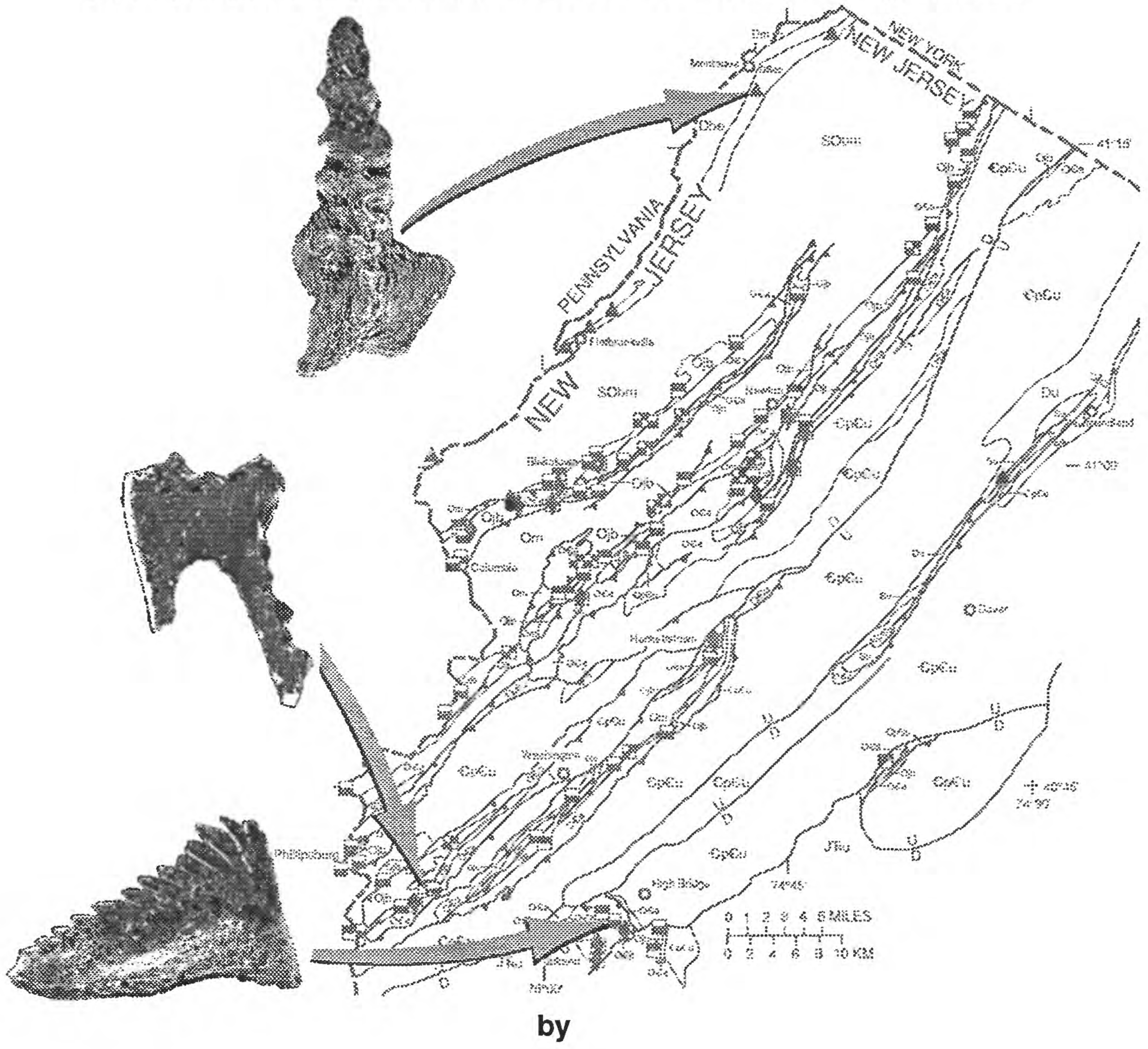

\section{Anita G. Harris, John E. Repetski, Nancy R. Stamm, and David J. Weary U.S. Geological Survey, MS 970, National Center, Reston, VA 22092}

\section{Open-File Report 95-557}

This report is preliminary and has not been reviewed for conformity with Geological Survey editorial standards or with the North American Stratigraphic Code. Any use of trade, product, or firm names is for descriptive purposes only and does not imply endorsement by the U.S. Government. 


\section{INTRODUCTION}

Conodonts are the microscopic toothlike hard parts of an extinct group of marine vertebrates having affinities to the jawless fishes (e.g., hagfish). Since the first discovery of a nearly complete conodont animal from the Lower Carboniferous of Scotland (Briggs and others, 1983), several other animals have been found (Mikulic and others, 1985; Aldridge and Theron, 1993). The name conodont applies to the animal as well as its only known hard parts. These hard parts, conodont elements, generally range from 0.1 to $1 \mathrm{~mm}$ in length, are composed of calcium phosphate, occur in a variety of shapes, even within a single animal (like the variety of tooth shape in many other vertebrates), and formed part of a feeding apparatus. Conodonts range from the Cambrian through Triassic (an interval of $~ 300$ million years), had worldwide distribution in most marine environments, and are one of the major groups of index fossils throughout their geologic range. An excellent summary of conodont taxonomy, biostratigraphy, and paleoecology is provided by Sweet (1988). Conodonts are also indices of thermal metamorphism (Epstein and others, 1977; Rejebian and others, 1987). The trace amount of organic matter sealed within these transparent skeletal elements visibly changes from pale yellow, to amber, light brown, dark brown, and black in the range of $50^{\circ}$ to $300^{\circ} \mathrm{C}$ as a result of carbonization. Above $300^{\circ} \mathrm{C}$, conodonts change from black, to gray, opaque white, and, finally, crystal clear as a result of carbon loss and recrystallization. These color changes, termed color alteration indices (CAI), have been reproduced and calibrated in the laboratory so that conodonts have become valuable geothermometers.

This report, exclusive of table 1 , is virtually identical to the conodont chapter in a volume on the paleontology of New Jersey (Repetski and others, 1995) to be published by the Geological Association of New Jersey. Space restrictions in that volume do not allow inclusion of the database for our paper. This database is presented here as table 1 and provides detailed geographic, stratigraphic, and biostratigraphic information for every conodont collection from New Jersey reposited in the U.S. Geological Survey conodont laboratory, Reston, Va.

\section{Previous Studies in New Jersey}

Conodonts are known only from northwestern New Jersey including at least 180 collections from about 90 localities that could be plotted separately on figure 1 (table 1). Only a few publications describe conodonts from New Jersey. The first such report was by Ethington and others (1958) on conodonts from Middle Ordovician siltstone in the Jutland klippe sequence (fig. 1, locs. HB-6, CH-1). Subsequently, Barnett (1965) described Middle Ordovician conodonts from the Jacksonburg Limestone; all of Barnett's localities have been re-collected in the course of geologic mapping (Drake and others, in press) and are included on figures 1 and 2. Barnett (1966, 1971, 1972), Epstein (1970), and Denkler and Harris (1988) reported on conodonts from uppermost Silurian and lowermost Devonian rocks in northwesternmost New Jersey, and Barnett $(1966,1971)$ described latest Silurian conodonts from the Green Pond Mountain area (fig. 1, loc. DO-1). Harris and others (1978) mapped conodont CAI values in Paleozoic rocks in the Appalachian basin including New Jersey, and Karklins and Repetski (1989) showed the distribution of selected Ordovician conodont faunas and their CAI values in the state. In addition, several abstracts and two undergraduate theses on Ordovician conodonts in New Jersey contain important stratigraphic and distribution data (e.g., Savoy, 1981).

In the past 20 years, conodonts have been used more than any other fossil group for correlation and to refine the age of lower Paleozoic marine rocks in New Jersey. Conodonts have been recovered chiefly from carbonate-bearing Upper Cambrian to Middle Ordovician and Upper Silurian and Lower Devonian rocks (fig. 3). Conodonts have also been identified on bedding planes of fine-grained siliciclastic rocks in the Cambrian and Ordovician Jutland klippe sequence where they are locally very abundant. Conodonts have been particularly important for correlation and geologic mapping of the Beekmantown Group, a lithologically repetitious and complexly deformed unit that generally lacks other fossils. Conodonts from New Jersey have been relatively little studied because they are mostly deformed and fractured (pl. I:A-C,H; pl. II:Y; pl. 


\title{
7.5'MINUTE QUADRANGLE ABBREVIATIONS
}

\author{
BA Bangor \\ BE Belvidere \\ BLR Blairstown \\ BL Bloomsbury \\ BR Branchville \\ BU Bushkill \\ $\mathrm{CH}$ Chester \\ CG Culvers Gap \\ DO Dover \\ EA Easton \\ FL Flatbrookville \\ HA Hackettstown \\ HBG Hamburg \\ HB High Bridge \\ LM Lake Maskenozha \\ MI Milford
}

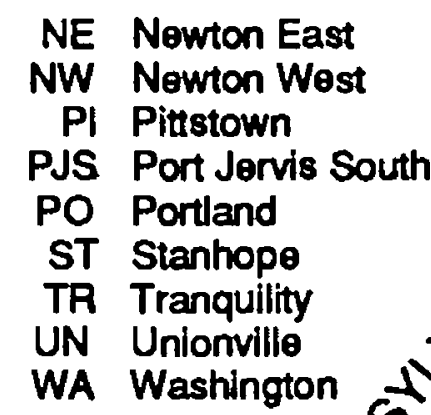

.

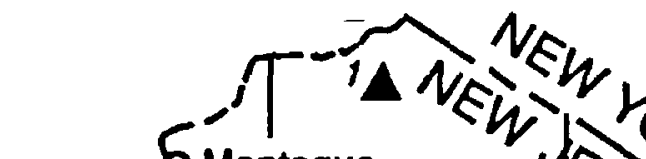<smiles>[Os+]</smiles>

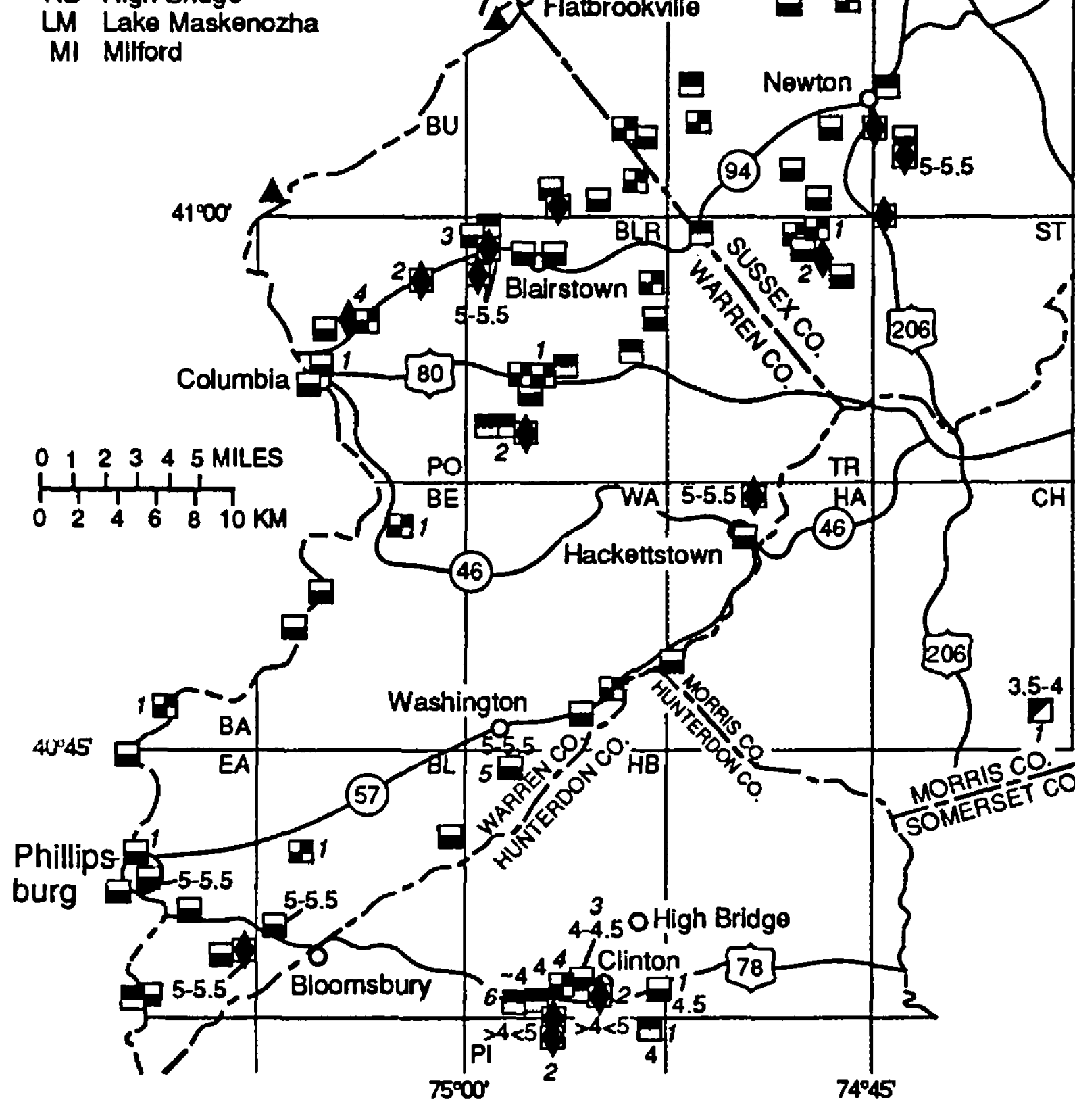

Figure 1. Index map of northwestern New Jersey showing conodont localities. See figure 2 for geologic setting.

III; V,X,Z). Most of the Paleozoic rocks in which they occur have undergone very lc 'v to low grade metamorphism, are complexly deformed, cleaved, and are relatively poorly exposed. Consequently, systematic collections from measured sections are difficult to obtain. In addition, most of the carbonate-bearing Paleozoic stratigraphic units in New Jersey, excluding the Stonehenge Formation and Jacksonburg Limestone, are poorly productive of conodonts (1-10 elements $/ \mathrm{kg}$ ). All of the above make biostratigraphic and taxonomic studies of conoronts in New 


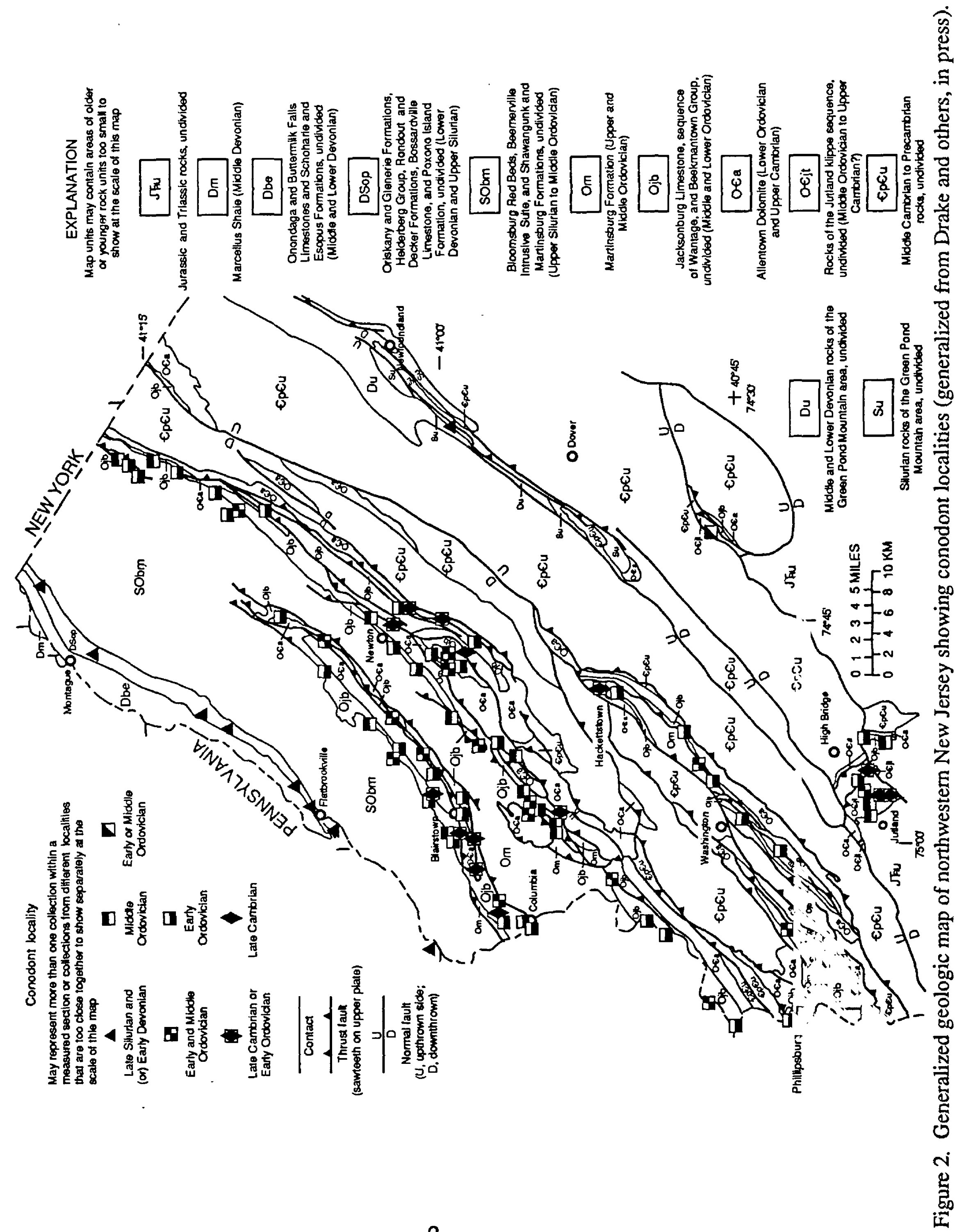


Jersey a difficult as well as long-term undertaking. This report includes collections made by many geologists in the last three decades, chiefly related to geologic mapping. Foremost arnong these are A.A. Drake, Jr., J.B. Epstein, P.T. Lyttle, and ourselves, U.S. Geological Survey, and R. Dalton, G.C. Herman, and D.H. Monteverde, New Jersey Geological Survey.

\section{STRATIGRAPHIC DISTRIBUTION OF CONODONTS IN NEW JERSEY}

\section{Upper Cambrian and Lower Ordovician Rocks}

\section{Allentown Dolomite (Upper Cambrian and Lower Ordovician?)}

The oldest stratigraphic unit to produce conodonts in New Jersey is the Allentown Dolomite (fig. 3). A limestone within the Allentown (fig. 1, loc. TR-2) yielded a single element of Proconodontus muelleri (pl. I:Z), indicating a latest Cambrian age. A few other Allentown localities also have produced a very few conodonts, but all are taxa that range from the Late Cambrian into the Early Ordovician.

\section{Beekmantown Group}

Stonehenge Formation (Upper Cambrian? and Lower Ordovician, lowe- Ibexian)

The Stonehenge Formation has produced the largest number of Early Ordovician conodonts from the New Jersey carbonate platform succession because the Stonehenge interval was intensively examined during recent geologic mapping and its depositional setting was more favorable for conodonts than other Beekmantown Group rocks. Our use of Stoneherge Formation, as well as Rickenbach Dolomite and Epler Formation, generally follows that of Hobson's (1963) regional stratigraphic study of the Beekmantown Group in southeastern Pennsylvania, except that the Stonehenge is now recognized in New Jersey (Drake and others, in press). During deposition of the Allentown Dolomite and Beekmantown Group, much of North America, including New Jersey, was a shallow marine carbonate platform with predoninantly shallow subtidal to supratidal depositional settings. Water temperature and salinity were undoubtedly elevated producing conditions unfavorable for abundant and diverse faunas. Some limestones (or dolomitized limestones) in the Stonehenge (and overlying Epler Forma tion) represent more "normal-marine" shallow subtidal conditions. These intervals generally produce indentifiable conodonts even though other organic remains are virtually absent. Othe- organisms may have once been present but their remains have not been preserved.

The lack of data near the gradational contact of the Stonehenge Formation with the underlying Allentown Dolomite, combined with the lack of agreement about the level for the Cambrian-Ordovician boundary, does not allow accurate placement of this contact; all assignable collections from the Stonehenge, however, are Ibexian (fig. 3). Collections from the earliest Ibexian Cordylodus proavus and $C$. intermedius Zones include both the name-bearing species as well as subzonal indicators such as Clavohamulus elongatus (pl. I:AF), Hirsutodontus simplex (pl. I:AC), and Clavohamulus hintzei (pl. I:AG).

Most limestones within the Stonehenge of New Jersey fall within the short interval of the Cordylodus angulatus Zone or the succeeding, geographically and stratigraphically ertensive, Rossodus manitouensis Zone. Collections from limestones within the $R$. manitouensis Zone can contain diverse as well as common to abundant conodonts ( 10-100 elements $/ \mathrm{kg})$. Besides the distinctive zonal index (pl. I:J-L), these collections usually include the tropical-cosmopolitan species Variabiloconus bassleri (pl. I:A-D), "Oistodus" triangularis (pl. I:M), and Scolopodus sulcatus (pl. I:H-I). Less common but important species within this zone are Acanthodus uncinatus (pl. I:E), Chosonodina herfurthi (pl. I:P), Clavohamulus densus (pl. I:R), Loxodus bransoni (pl. I:S), and "Paltodus" spurius (pl. I:T). The Stonehenge conodont fauna? like those in the rest of the Beekmantown Group, are shallow-water, tropical to low-temperate species associations typical of the North American Midcontinent Faunal Realm. 


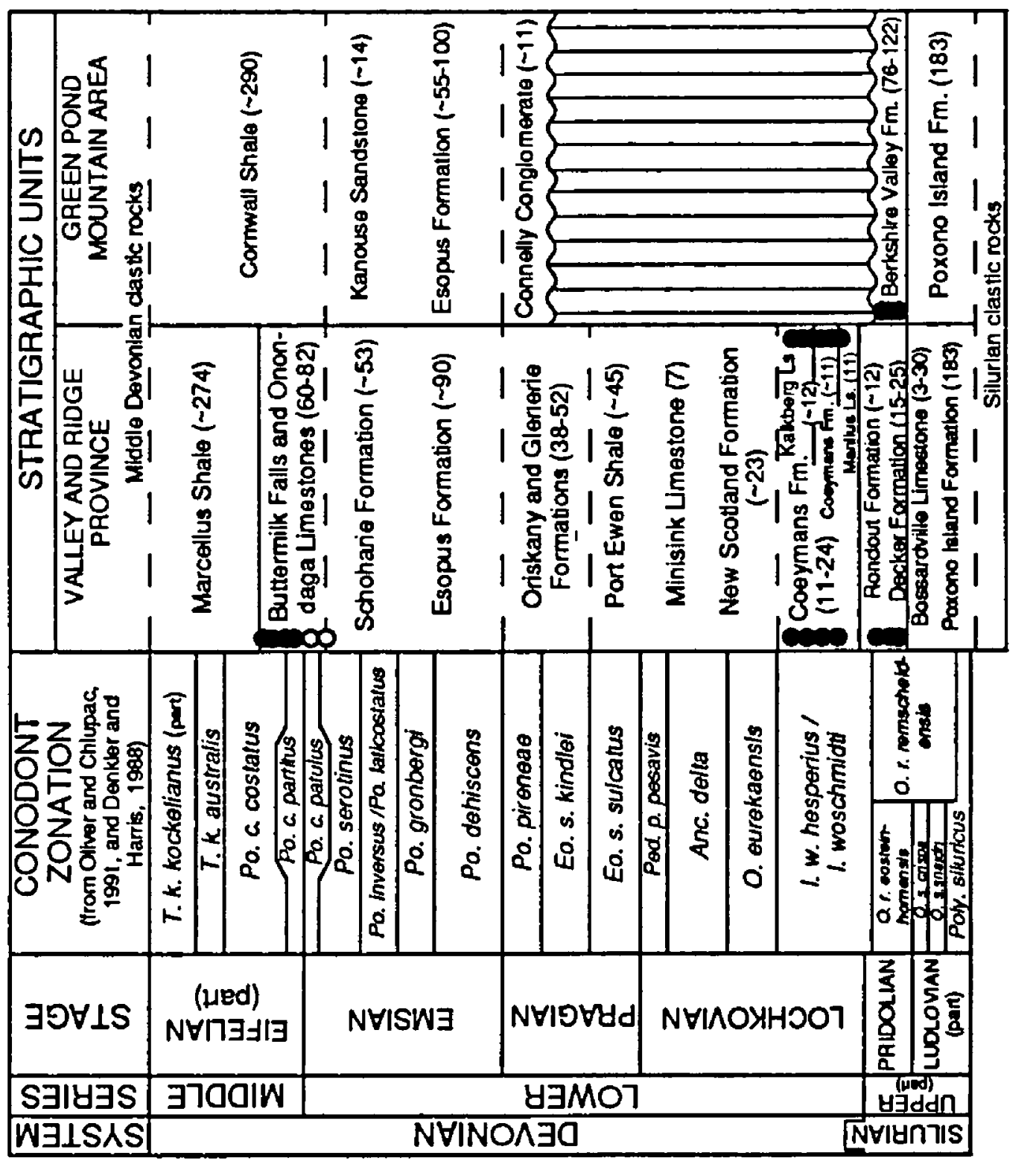

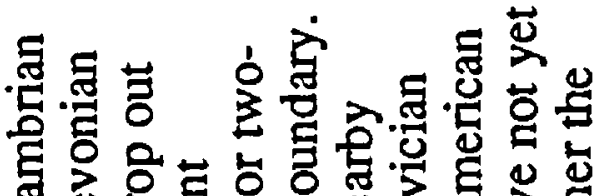

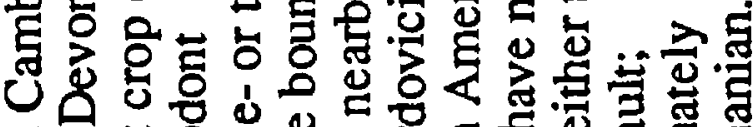

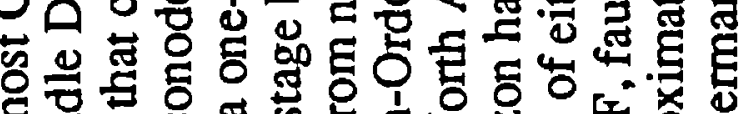
7.

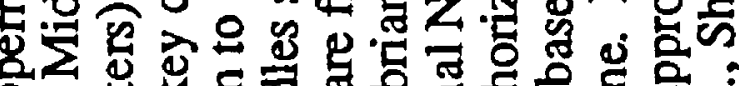
둥 은 范 연.

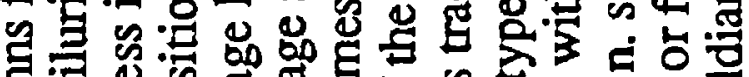

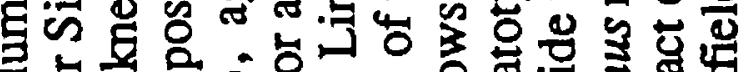

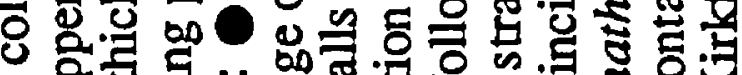

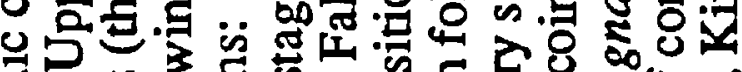

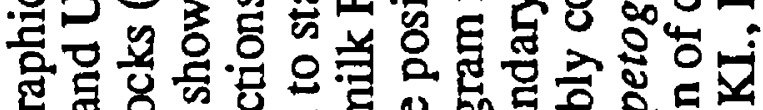
대은

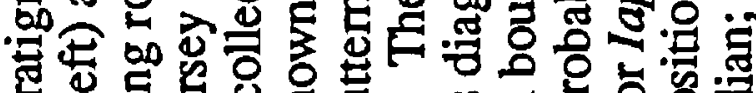

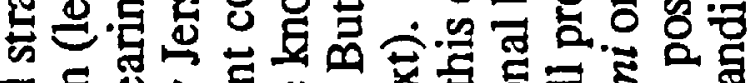

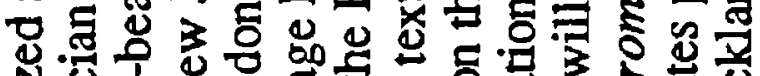

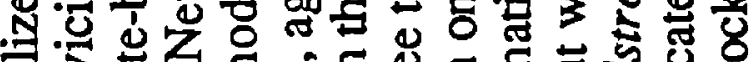

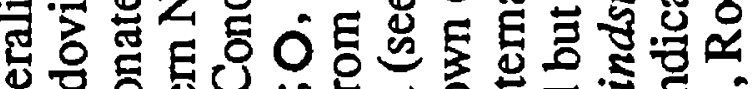

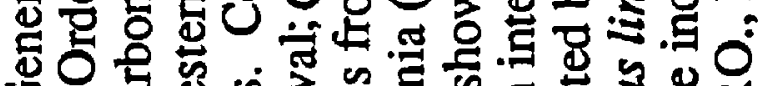
0 记

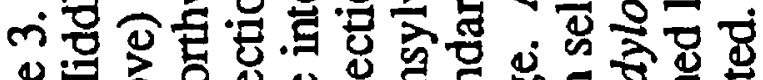

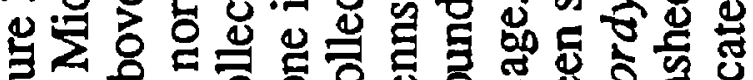

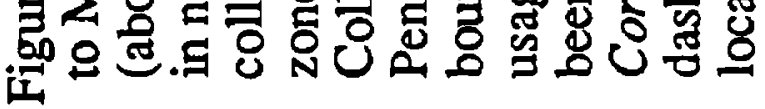

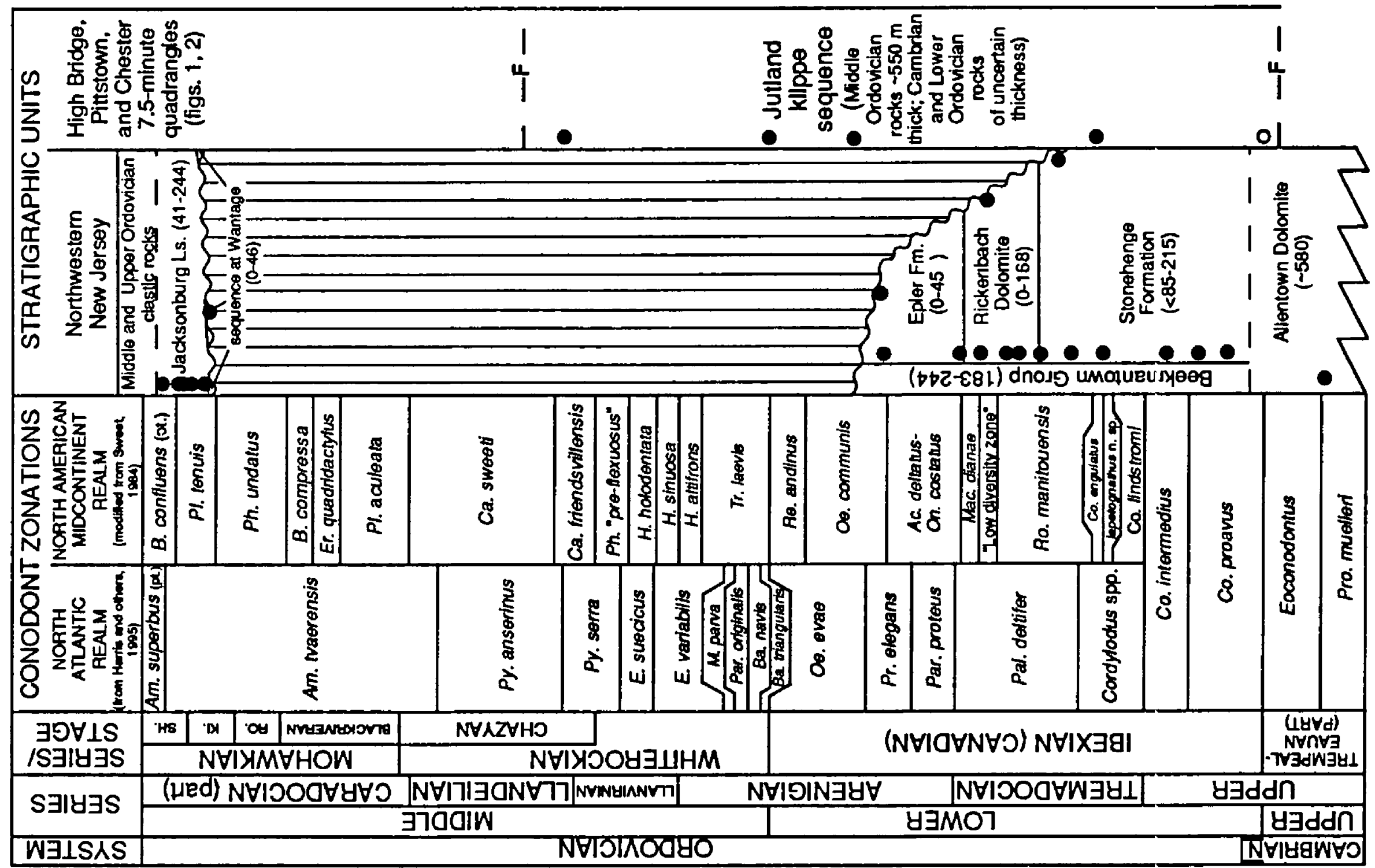


Rickenbach Dolomite (Lower Ordovician, lower and middle Ibexian)

The lower contact of the Rickenbach Dolomite is gradational with the Stonehenge

Formation and occurs within the R. manitouensis Zone. Thus, it is difficult or impos rible to distinguish isolated dolostone outcrops of most of the Rickenbach from much of the upper part of the Stonehenge either lithically or faunally. Generally, however, the dolostones of the Rickenbach represent more restricted depositional environments so that, if they yield conodonts, the faunas are less abundant and less diverse than those of the Stonehenge.

The upper part of the Rickenbach contains species of the "Low diversity zone" and the Macerodus dianae Zone (fig. 3). Other than rare elements of $M$. dianae (pl. II:X,Y), this relatively thin interval usually produces only rather morphologically generalized conodonts sucl as Oneotodus simplex and Eucharodus spp., as well as Colaptoconus quadraplicatus (pl. II:AG) and Striatodontus? prolificus (pl. II:AD,AE).

\section{Epler Formation (Lower Ordovician, middle and upper Ibexian)}

The Epler Formation, gradational upward from the Rickenbach Dolomite, is mostly dolostone, but does contain some limestones that help distinguish these formations. The contact is within the $M$. dianae Zone (fig. 3). Collections from limestones low in the formation may contain rather diverse faunas, but abundance is generally low. Characteristic species include $M$. dianae (pl. II:X, Y), Protopanderodus leei (pl. II:AB,AC), Scolopodus floweri (pl. II:U-W), as well as Colaptoconus quadraplicatus and Eucharodus parallelus.

Very little is known about the distribution of conodonts in the middle and upper parts of the Epler in New Jersey because these strata have not been systematically sampled. A ferv isolated samples containing Diaphorodus delicatus ( $\mathrm{pl}$. II:O) indicate the formation extends at least into the late Ibexian Oepikodus communis Zone. In addition, O. communis (pl. II:Q) was reçvered from a redeposited cobble of Epler lithology within the Jacksonburg Limestone in an area from which the Epler was eroded (Savoy, 1981).

\section{Middle Ordovician}

\section{Jacksonburg Limestone (late Middle Ordovician)}

The Jacksonburg Limestone disconformably overlies all of the Beekmantown Group formations in northwestern New Jersey, as these rocks were exposed and eroded during the early Middle Ordovician. Savoy (1981) documented the presence of conodonts ranging in age from early to late Ibexian (to perhaps early Whiterockian) in carbonate cobbles incorporated' within the basal and lower Jacksonburg. In addition, isolated exposures of dolomitic to shaly carbonate rocks lie immediately beneath the Jacksonburg in some areas. These rocks, mapped and termed the sequence at Wantage by Monteverde and Herman (1989) and Drake and others (ir press), probably are remnants of a unit that filled topographic lows on the eroded Beekmanto'wn surface during initial late Middle Ordovician transgression. Some samples from this unit have produced Phragmodus undatus among other species. This species and the position of the sequence at Wantage beneath the Jacksonburg indicate a Rocklandian or Kirkfieldian age.

The limestones of the Jacksonburg generally contain abundant and diverse shelly fossils and probably formed in normal-marine subtidal conditions. These limestones also produce the largest conodont yields of all New Jersey carbonate rocks. Most Jacksonburg samples contain the North American Midcontinent Faunal Realm species Phragmodus undatus (pl. III:V-Z), Plectodina tenuis (pl. III:R,S), Aphelognathus politus (pl. III:M-Q), as well as pandemic species such as Panderodus gracilis and Drepanoistodus suberectus (pl. III:AC). All these species are rather longranging, but some constrain the lower limit of the formation to an age no older than late Middle Ordovician (Kirkfieldian Stage) Plectodina tenuis Zone (fig. 3). As noted by Barnett (1965), at some localities the Jacksonburg faunas include species characteristic of the North Atlantic Faunal Realm together with North American Midcontinent species. This probably indicates a more offshore, open-marine setting for these more eastern localities (e.g., fig. 2, loc. PI-1). North Atlantic Realm species found in the Jacksonburg include Icriodella superba (pl. III:A-D), Rhodesognathus elegans (pl. III:L), and Amorphognathus superbus (pl. III:F,G). The presence of 
A. superbus is significant because it ties the Jacksonburg into the cosmopolitan North Atlantic zonation (fig. 3). The age of the upper part of the Jacksonburg is not well constrained: graptolites in the lower part of the overlying Martinsburg Formation indicate an age no younger th an early Edenian (Drake and others, in press).

Jutland klippe sequence

Very different conodont faunas occur sporadically in the mostly siliciclastic rocks of the Taconic-type klippen near Clinton (Jutland klippe) and near Chester (Peapack klippe, but included in the Jutland klippe sequence), New Jersey (figs. 2, 3). All faunas, both conodont and graptolite, known from these areas, as well as the sedimentology, point to deposition in continental slope to oceanic basin environments (see also Drake, 1969; Perissoratis and others, 1979). All conodonts known from the klippe sequence thus far are cosmopolitan North Atlantic Realm or pandemic species.

Conodonts are locally abundant on bedding surfaces of siltstone and fine-grained sandstone in the Jutland klippe sequence. These include mostly elements of Periodon aculeatus (pls. II:F, III:AB) and a few other long-ranging taxa that give an overall age of early to middle $\mathrm{M}$ : Idle Ordovician. However, two specimens of Pygodus, a biostratigraphically important genus, known from the sequence (Ethington and others, 1958, and this report, pl. III:AA), restrict the age to the Llanvirnian and (or) Llandeilian. Some new collections made by D.C. Parris (N.J. State Museum), from a lower stratigraphic level contain Early Ordovician graptolites and ab"undant conodont elements of Paracordylodus gracilis (fig. 1, loc. HB-3). These conodonts are best assigned an early or early middle Arenigian (early late Ibexian) age.

Some collections of calcareous rocks from the Jutland klippe sequence have yielded older conodonts. The oldest faunas span the Cambrian-Ordovician boundary (fig. 3), and include Eoconodontus notchpeakensis (pl. I:X), and species of the protoconodont Phakelodus (pl. I:V). Somewhat younger samples contain species of the cosmopolitan genus Cordylodus ant are assignable to the lower Ibexian or lower Tremadocian (pl. II:G,H). A calcareous sample from the Peapack klippe (fig. 1, loc. CH-1) contains abundant Periodon flabellum (pl. II:A-E) and other species (pl. II:I-M), and is of latest Early or earliest Middle Ordovician age.

\section{Upper Silurian and Lower Devonian Rocks}

\section{Valley and Ridge province}

\section{Decker Formation (Upper Silurian, upper Pridolian)}

The Decker Formation is the oldest Silurian unit to produce conodonts (fig. 3). No conodonts have been recovered from the underlying Bossardville Limestone in New Jersey and samples from Pennsylvania have produced only indeterminate conodont fragments (Denkler, 1984). The Poxono Island Formation underlies the Bossardville and contains some carbonate beds, but it has not produced conodonts either in New Jersey or nearby Pennsylvania (Denkler, 1984 and A.G. Harris, unpublished data). Barnett (1971) showed the distribution of conodonts in samples from the Decker Formation at loc. PJS-1 (fig. 1). Barnett's data indicate conodonts are common (11 elements $/ \mathrm{kg}$ ) but diversity is low; Ozarkodina remscheidensis makes up more than $98 \%$ of the fauna and Oulodus elegans is the only other conodont on the basis of our miltielement analysis of Barnett's form-element taxonomy. These two species also occur in the Decker in Pennsylvania (Denkler and Harris, 1988). The Decker contains a relatively diverse mecrofauna including brachiopods, corals, mollusks, and pelmatozoans. Fossils and sedimentary structures indicate a high-energy, shoal-water depositional setting. The low diversity of conodonts suggests normal-marine conditions were intermittent or that physical and chemical barriers elsewhere in the Appalachian basin kept less eurytopic conodont species from reaching New Jersey.

Oulodus elegans indicates the Decker Formation is no older than late Ludlovian and no younger than latest Pridolian. Regional stratigraphic relationships indicate the Decker is late Pridolian. 


\section{Rondout Formation (Upper Silurian and Lower Devonian, upper Pridol ’n and lower Lochkovian)}

The Rondout Formation consists of laminated, mud-cracked, argillaceous cart onate mudstone and dolostone containing one widespread, thin biostromal bed. The Rondcut formed in a restricted lagoon setting (Epstein and others, 1967). Only one sample (fig. 1, loc. PJS-1) from the lower part of the formation in New Jersey produced conodonts (Barnett, 1971). Abundance and species composition is the same as in the Decker. Oulodus elegans restricts the lower part of the Rondout to the Late Silurian. The Silurian-Devonian boundary was placed in the middle of the Rondout Formation on the basis of sequence stratigraphy and regional conodont biostratigraphy and biofacies by Denkler and Harris (1988). Earlier, Barnett (1972) positioned this boundary at the base of the overlying Manlius Limestone in New Jersey using comparative morph ometric analysis of $\mathrm{Pa}$ elements of Ozarkodina remscheidensis from New Jersey, Nevada, and Czechoslovakia. We believe changes in the shape of $\mathrm{Pa}$ elements of $O$. remscheidensis, a longranging and variable species, reflect recurrent environmental responses, are not evolutionary, and should not be used for long-distance correlation.

\section{Manlius Limestone (Lower Devonian, I. woschmidti Zone, lower Lochkovian) Conodonts are common ( 10 elements $/ \mathrm{kg})$ in the Manlius Limestone in New Jersey} (Barnett, 1966; Epstein, 1970; A.G. Harris, U.S. Geological Survey collections) and only slightly more diverse than in underlying Silurian rocks. Ozarkodina remscheidensis (pl. IV:E, G-I) continues to make up at least $98 \%$ of the collections indicating intermittently restricted conditions. Oulodus cristagalli and Belodella devonica are rare. One specimen of Icriodus woschmidti, an auxiliary guide to the base of the Devonian, was found $2.6 \mathrm{~m}$ below the top of the formation at loc. PJS-1 (Denkler and Harris, 1988) confirming the Devonian age based on regional relationships. Southwest of Montague, N.J., beds correlative to the Manlius become increasingly quartzose and are included in the more heterogeneous Coeymans Formation (fig. 3; Epstein and othars, 1967).

\section{Coeymans Formation (Lower Devonian, I. woschmidti Zone, lower Lcrhkovian)}

The Coeymans Formation yields more abundant ( 20 elements $/ \mathrm{kg})$ and possitly more diverse conodonts than any other uppermost Silurian or lowermost Devonian unit in I'ew Jersey (Barnett, 1966, 1971; Epstein, 1970). The Coeymans includes bioclastic grainstone, packstone, and lesser wackestone that contain varying amounts of quartz silt and sand as well as biorudite and calcareous quartz-pebble conglomerate. The biorudites produce fewer conodonts thar other facies in the formation. The conodont fauna of the Coeymans (using data in Barnett, 1966, and Epstein, 1970) consists of Ozarkodina remscheidensis (76\%) (pl. IV:A-D, F), Icriodus woschmidti (12\%) (pl. IV:Q-V), Oulodus cristagalli (9\%) (pl. IV:K-O), Belodella devonica (2\%) (pl. IV:P), Pseudooneotodus beckmanni ( $1 \%$ ) (pl. IV:J), and 1 specimen of Panderodus unicostatus (Branson and Mehl). Sedimentary structures and fossils indicate the Coeymans formed in a highto moderate-energy depositional setting accounting for the increase in icriodids and oulodids.

\section{Kalkberg Limestone (Lower Devonian, I. woschmidti Zone, lower Lochkovian)}

The Kalkberg Limestone extends into New Jersey only as far southwest as the. Lake Maskenozha quadrangle (fig. 3; Epstein and others, 1967). The Kalkberg is like the underlying Coeymans Formation, but is more argillaceous and contains more chert. Nevertheles . the Kalkberg produces nearly the same conodont species in the same order of abundance as the Coeymans (Epstein, 1970). The presence of Icriodus woschmidti restricts the formation to the earliest Lochkovian. The Kalkberg was deposited seaward of the Coeymans in slightly deeper water.

\section{Post-Kalkberg Limestone Devonian rocks}

No conodonts have been reported from Devonian rock units younger that the Kalkberg Limestone in northwestern New Jersey. Of these units (fig. 3), the Glenerie Formation and Buttermilk Falls Limestone have the best potential for conodonts. Common to abundant conodonts have been recovered from measured sections of the Buttermilk Falls in its type area near East Stroudsburg, $\mathrm{Pa}$. ( lat. $41^{\circ} 00^{\prime}$, long. $75^{\circ} 08^{\prime}-11^{\prime}$ ) (A.G. Harris, written communication, 1982 , to 
J.B. Epstein; U.S. Geological Survey collections 10635-SD to 10642-SD). The conotonts indicate the lower one-third of the formation is no older than the serotinus Zone and the remainder of the formation represents the partitus and costatus Zones. In the type area, the Tiogs Ash Bed is $9 \mathrm{~m}$ below the top of the Buttermilk Falls Limestone. The Tioga is known to lie within the costatus Zone at many localities in the Appalachian basin (e.g., Klapper and Johnson, 1980).

\section{Green Pond Mountain area}

A narrow belt of Paleozoic rocks lies within the New Jersey Highlands, east of the Valley and Ridge province (fig. 2). Barnett $(1966,1971)$ described conodonts from carbonate-bearing uppermost Silurian and lowermost Devonian rocks at one locality in the Green Pond Mountain area of New Jersey (fig. 1, loc. DO-1). Three large samples from fossiliferous limestone treds in the Berkshire Valley Formation produced only Ozarkodina remscheidensis in low numbers $(4 / \mathrm{kg})$. On the basis of regional stratigraphic relations, Barnett $(1966,1971)$ considered the Berkshire Valley correlative to the Decker and Rondout Formations of the Valley and Ridge province.

\section{CONODONT COLOR AND TEXTURAL ALTERATION}

Most conodonts from New Jersey have CAI values of 5 and, more rarely, higl or (fig. 1 and table 1) indicating they reached at least $300^{\circ} \mathrm{C}$ and burial depths of at least $10 \mathrm{~km}$. Values of 4.5 to as low as 3.5-4 are limited to the High Bridge, Pittstown, and Chester 7.5-mint te quadrangles (fig. 1 and table 1), chiefly in the Jutland klippe sequence although conod nnts from the Beekmantown Group and Jacksonburg Limestone in the same area have similarly low CAI values (fig. 1, locs. PI-1 and HB-1-3 and fig. 2). The data suggest that all these rocks lie structurally above and were never as deeply buried as nearby parautochthonous correla tive strata with CAI values of 5 and 5-5.5 (e.g., fig. 1, loc. HB-5) as well as considerably younger Upper Silurian and Lower Devonian rocks with CAI values of 5 at least $40 \mathrm{~km}$ to the northwest.

Most conodonts from New Jersey are poorly preserved texturally, particularly specimens from dolostones in which saline pore waters probably promoted surface corrosion and recrystallization of conodonts during diagenesis. In limestones, recrystallization of conodonts generally begins at CAI 5. Because even the youngest conodonts from New Jersey have CAI values of 5, they too show varying degrees of recrystallization depending on the texture and composition of their host rock (see pls. I-IV). Conodonts from porous rocks such as grainstones may be more recrystallized than those from more poorly sorted rocks. For example, the specimen shown in plate IV:A is from a wackestone and is less recrystallized than the specimen shown in plate IV:K from a grainstone/packstone. At the temperatures associated with CAI values of 5 or higher, carbonate grains may anneal to the surface of conodonts. When such conodonts are freed from limestone by dissolving the rock in a weak acid solution, the conodonts often bear the imprint of annealed carbonate grains on their surface (e.g., pls. I:P, IV:R) even though these grains have dissolved. Conodonts extracted from dolomitic limestone, such as the Epler Formation, or quartzose carbonate rocks, such as the Coeymans Formation, generally retain the insoluble dolomite rhombs (e.g., pl. II:P,AH) or quartz grains on their surface (e.g., pl. IV:C, T). 


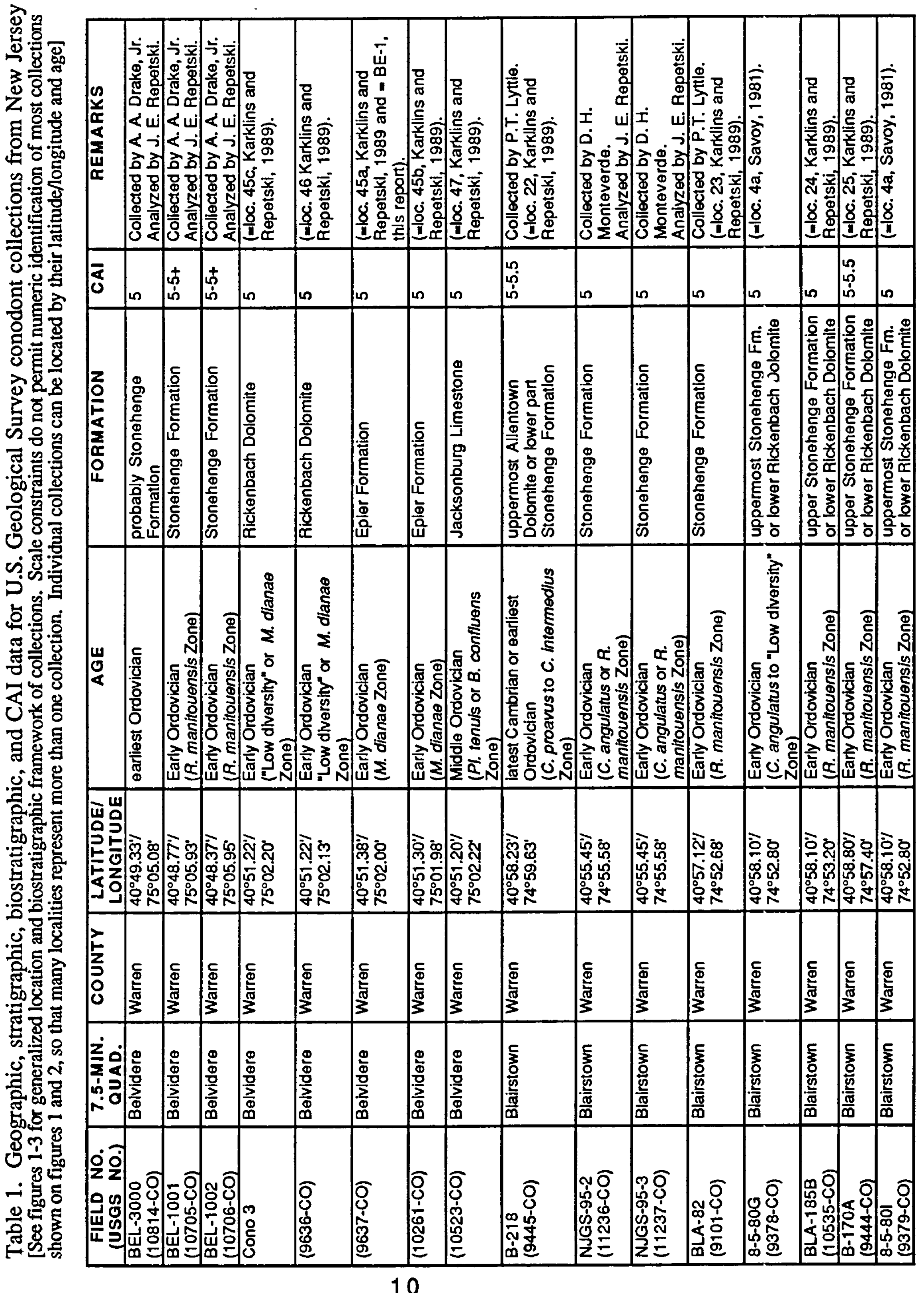




\begin{tabular}{|c|c|c|c|c|c|c|c|c|c|c|c|c|c|c|c|}
\hline 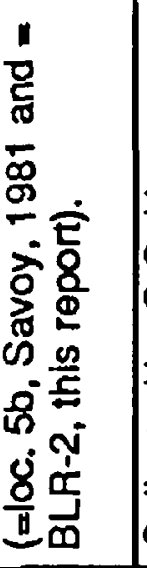 & 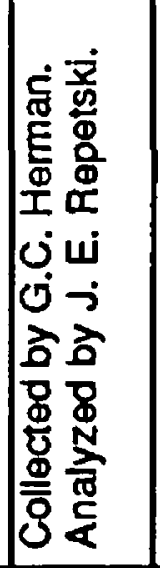 & 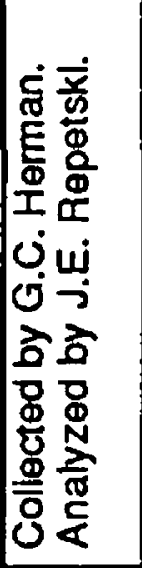 & 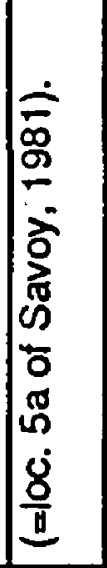 & 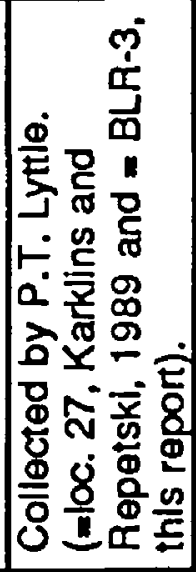 & 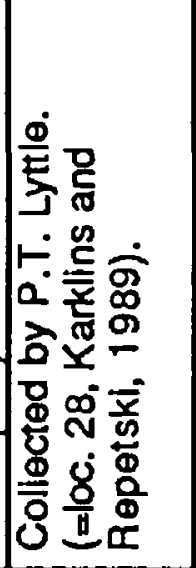 & 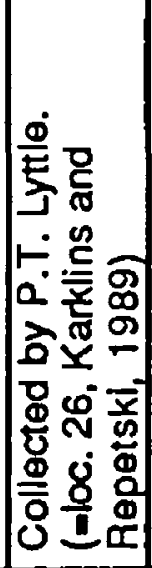 & 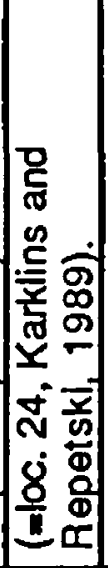 & 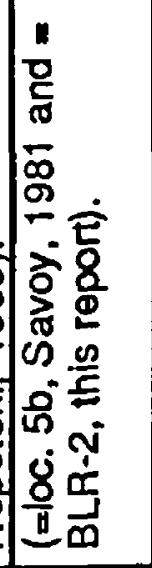 & 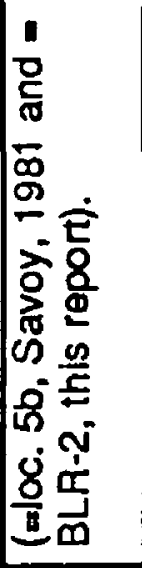 & 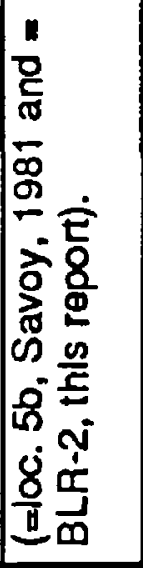 & 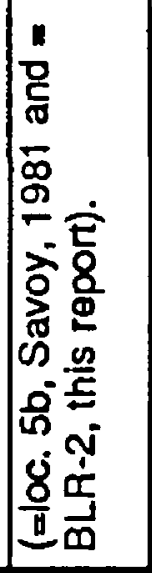 & 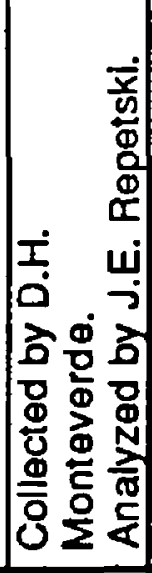 & 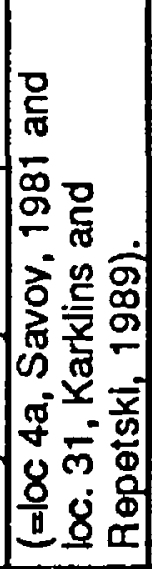 & 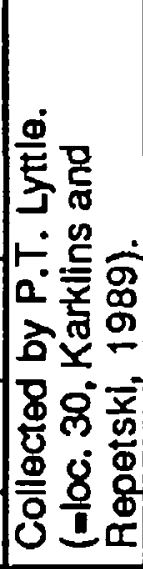 & 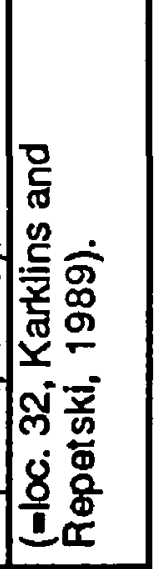 \\
\hline ת & n & n & م & مـ & תم & N & م & م & (n) & (1) & w & ns & 10 & 10 & م6 \\
\hline 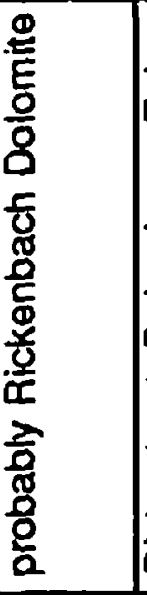 & 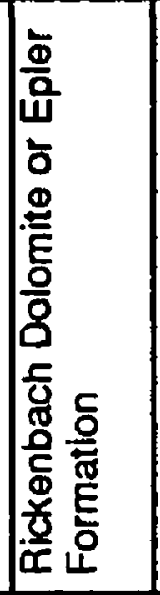 & 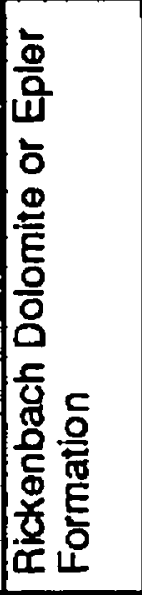 & 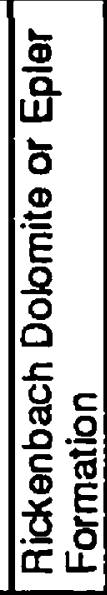 & 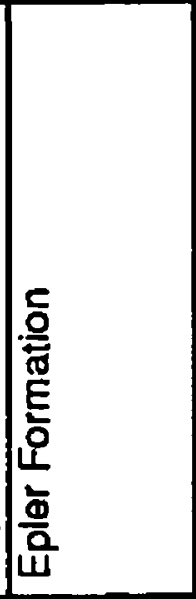 & 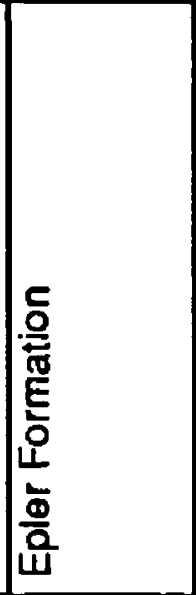 & 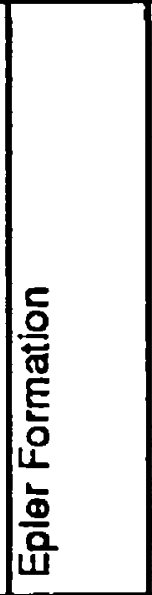 & 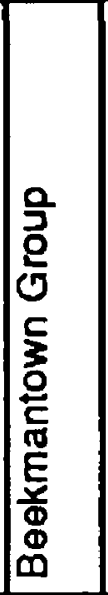 & 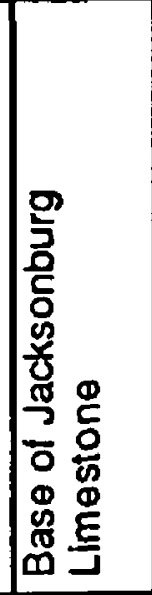 & 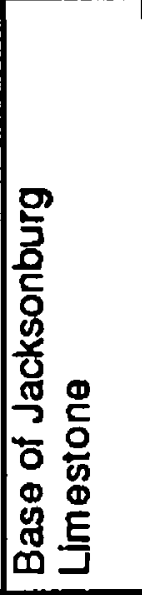 & 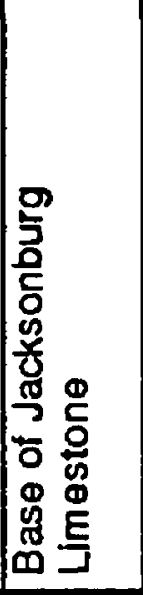 & 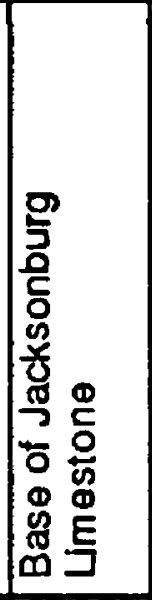 & 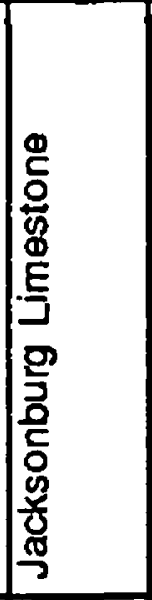 & 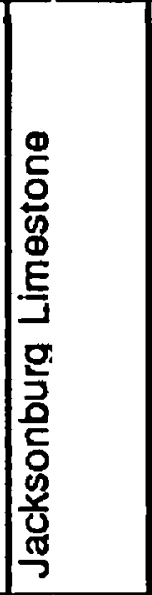 & 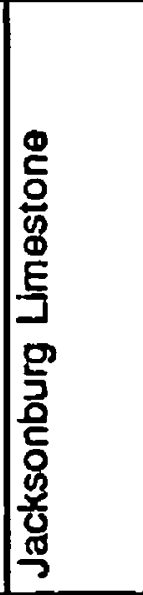 & 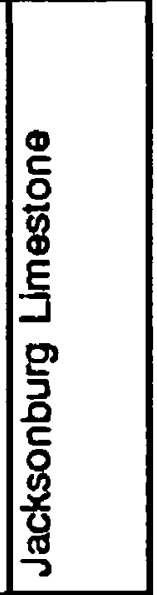 \\
\hline 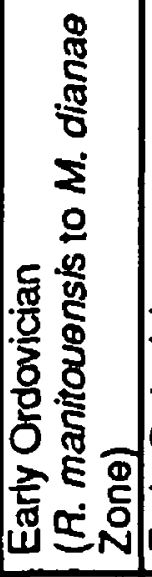 & 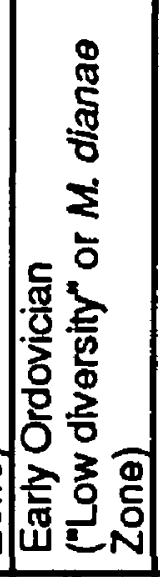 & 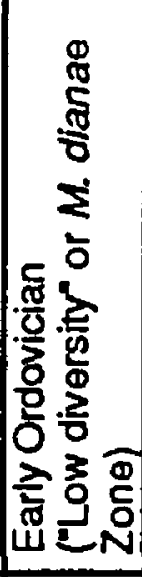 & 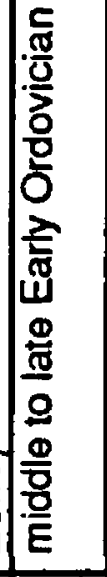 & 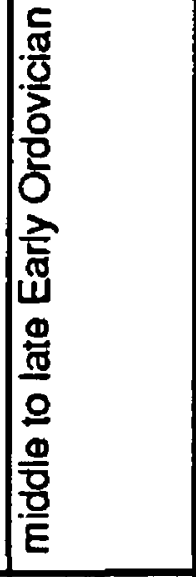 & 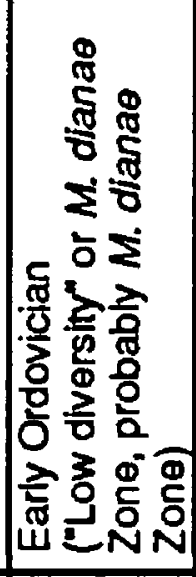 & 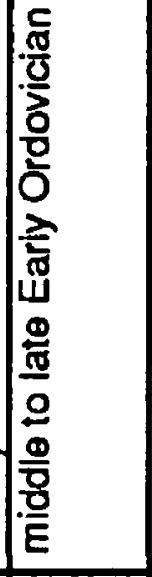 & 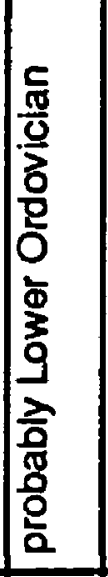 & 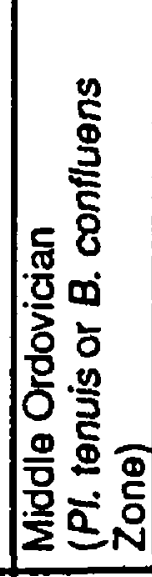 & 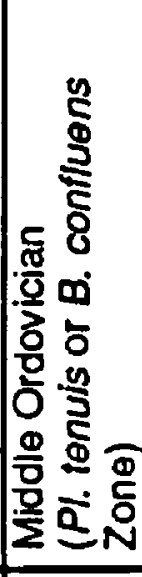 & 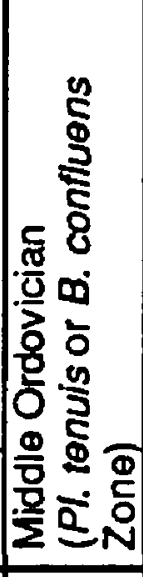 & 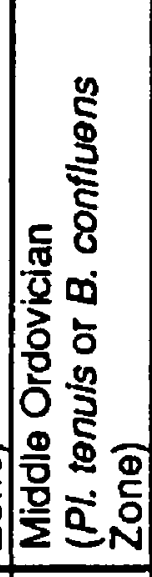 & 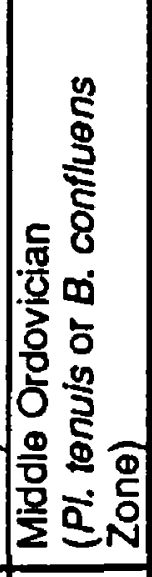 & 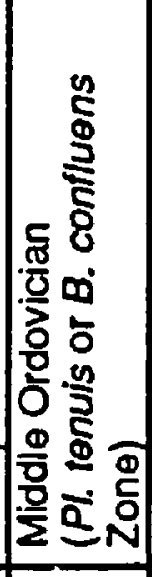 & 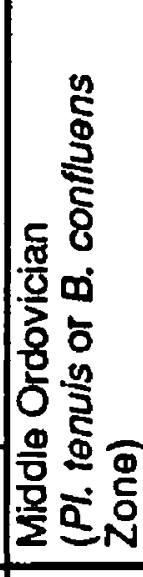 & 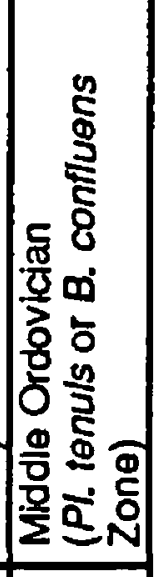 \\
\hline 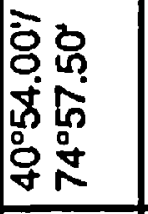 & 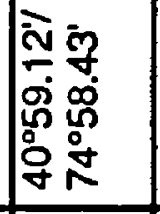 & 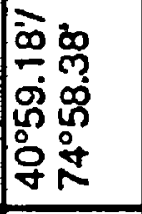 & 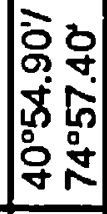 & 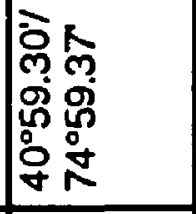 & 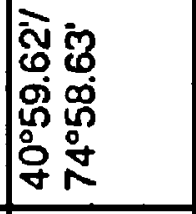 & 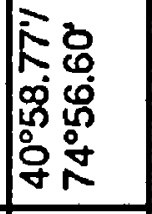 & 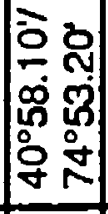 & 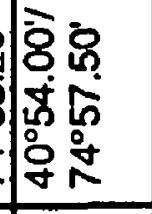 & 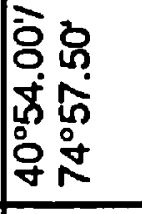 & 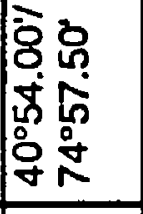 & 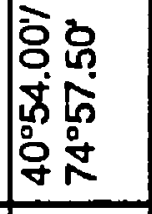 & 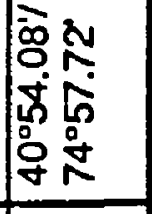 & 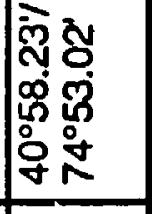 & 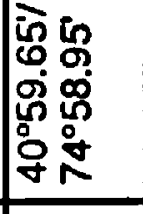 & 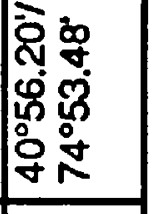 \\
\hline 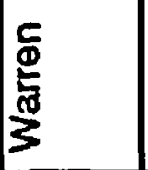 & 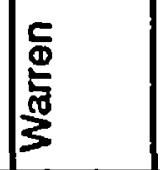 & 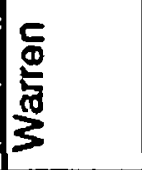 & 点 & 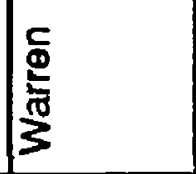 & 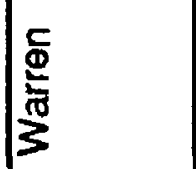 & 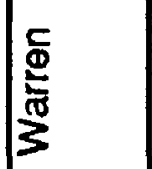 & 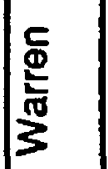 & 点 & 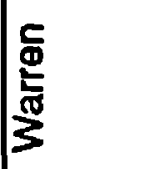 & 离 & 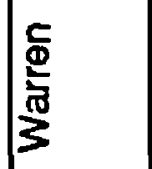 & 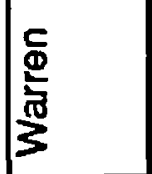 & 点 & 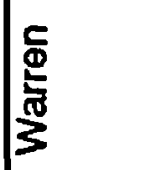 & 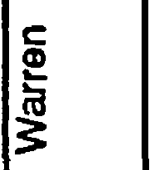 \\
\hline 占 & 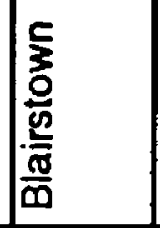 & $\begin{array}{l}\frac{5}{3} \\
\frac{0}{0} \\
\frac{5}{\frac{5}{\pi}} \\
\frac{\pi}{0} \\
\end{array}$ & $\mid \frac{c}{\frac{5}{0}}$ & 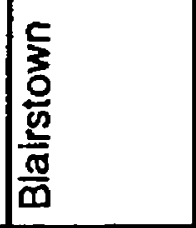 & $\frac{c}{\frac{c}{2}}$ & 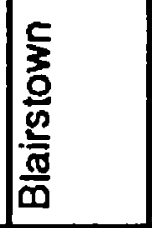 & 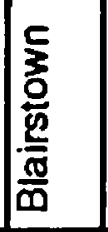 & 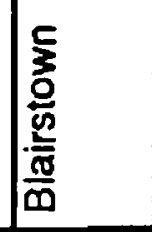 & 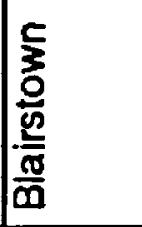 & $\mid \frac{5}{3}$ & 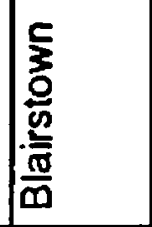 & 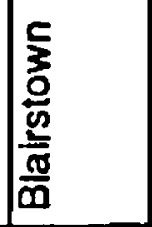 & $\frac{\frac{c}{3}}{\frac{0}{\frac{2}{6}}}$ & 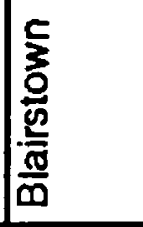 & $\frac{\frac{5}{5}}{\frac{5}{\frac{2}{0}}}$ \\
\hline 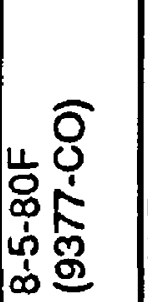 & 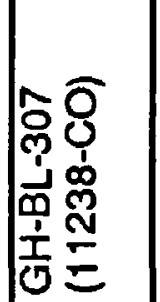 & 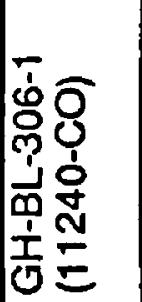 & 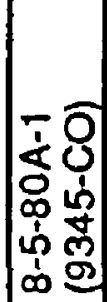 & 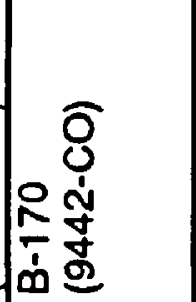 & 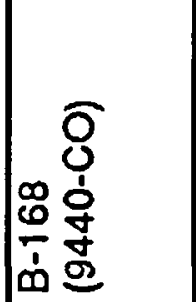 & 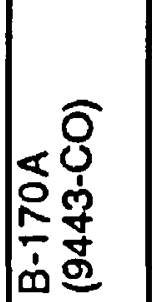 & $\mid$\begin{tabular}{ll}
$\varangle$ & 0 \\
$\infty$ & 0 \\
\hdashline & 0 \\
\hdashline & 0 \\
1 & 0 \\
& 0 \\
\end{tabular} & 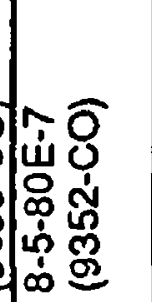 & 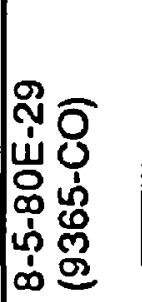 & 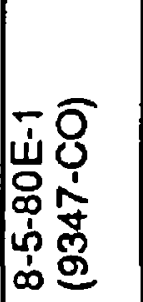 & 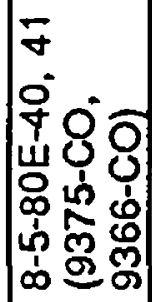 & 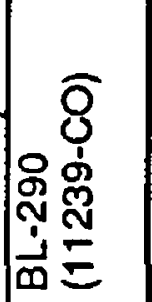 & 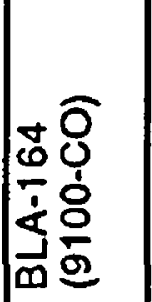 & 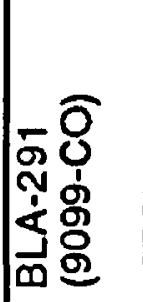 & 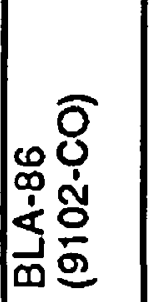 \\
\hline
\end{tabular}




\begin{tabular}{|c|c|c|c|c|c|c|c|c|c|c|c|c|c|c|c|}
\hline 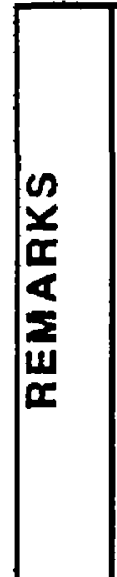 & 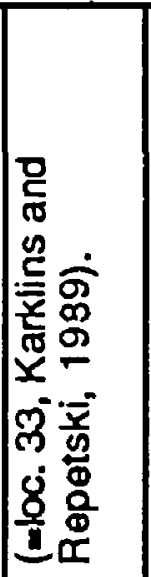 & 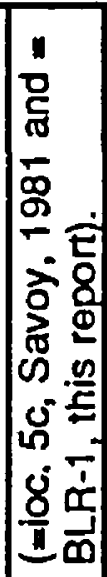 & 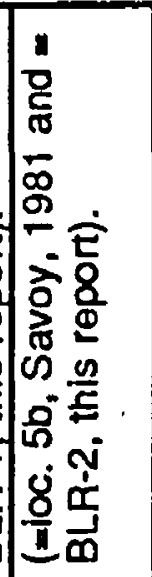 & 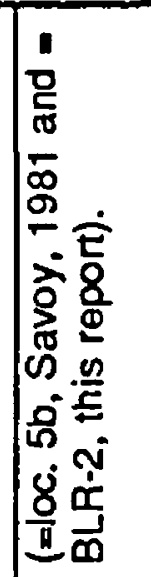 & 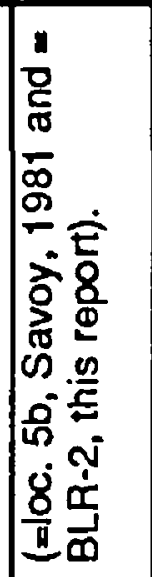 & 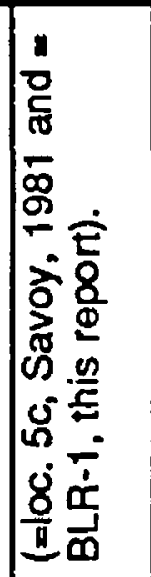 & 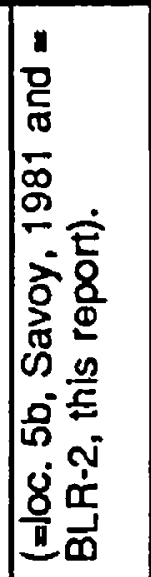 & 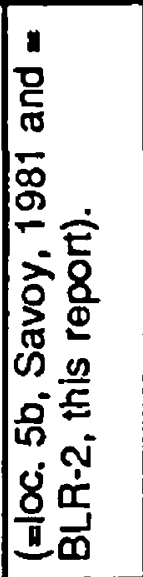 & 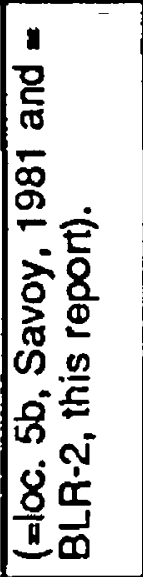 & 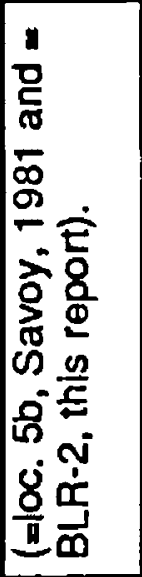 & 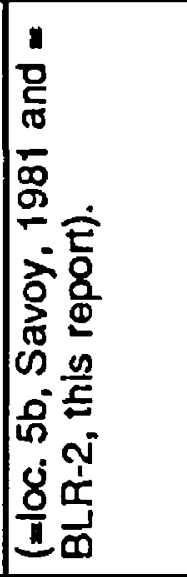 & 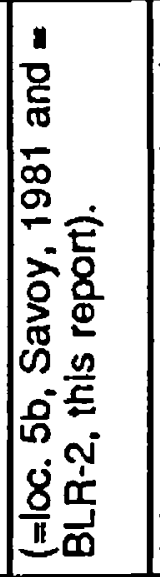 & 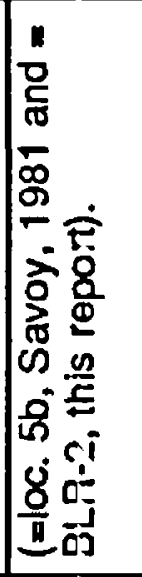 & 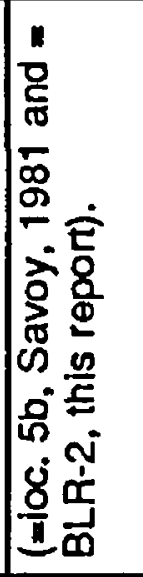 & 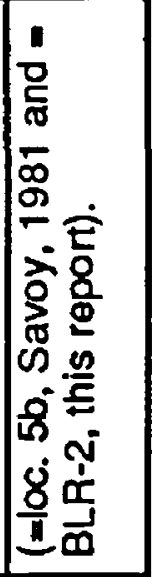 \\
\hline | & م & م & مـ & n & م & in & 10 & م & $\omega$ & n & $\omega$ & 10 & 10 & 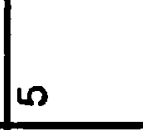 & ما \\
\hline 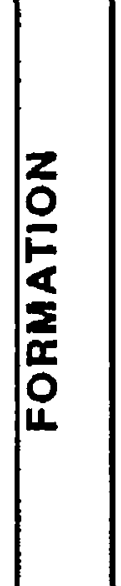 & 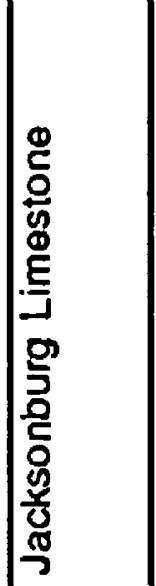 & 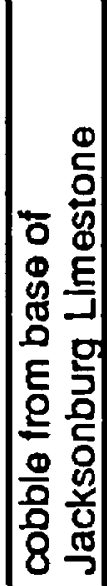 & 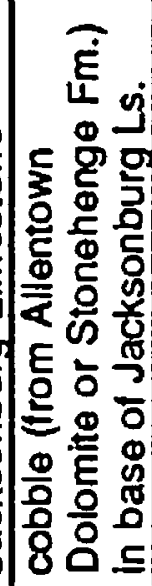 & 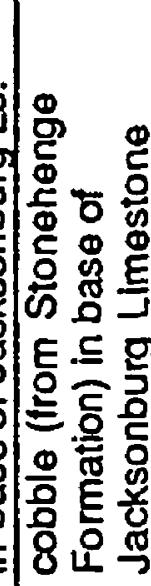 & 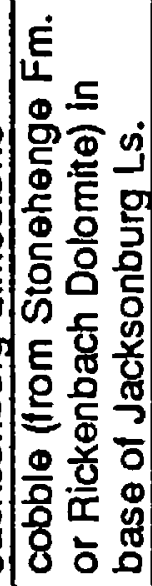 & 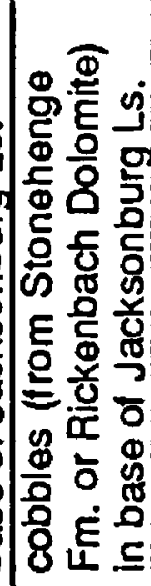 & 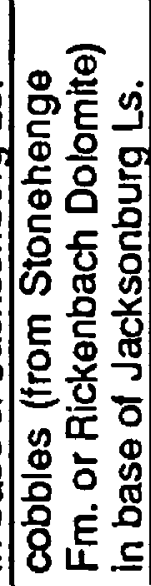 & 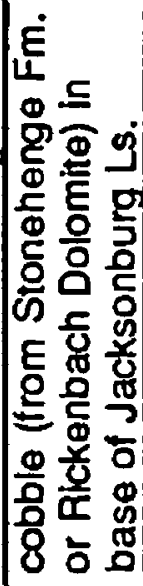 & 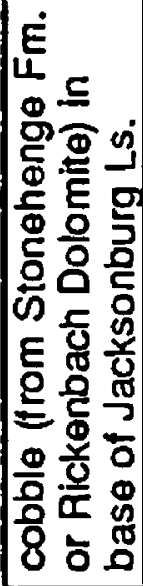 & 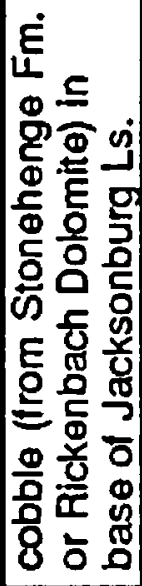 & 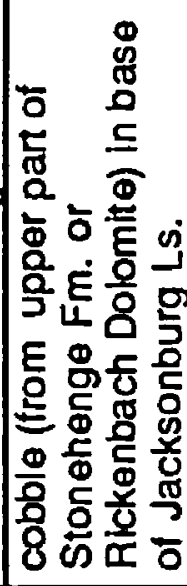 & 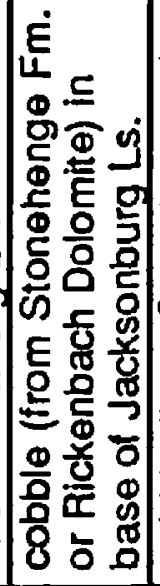 & 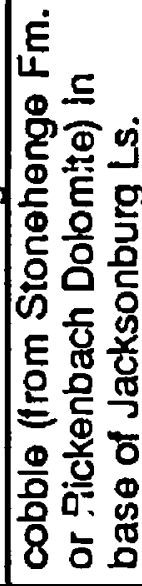 & 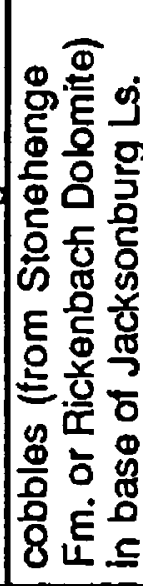 & 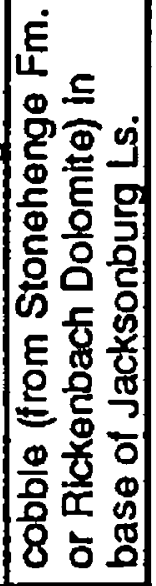 \\
\hline$\underset{0}{W}$ & 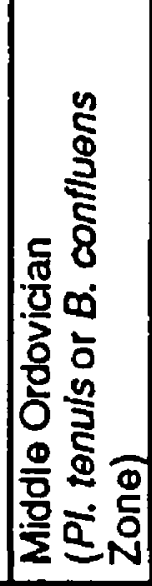 & 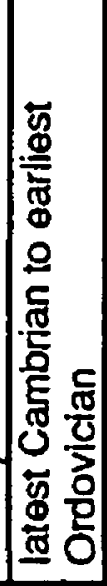 & 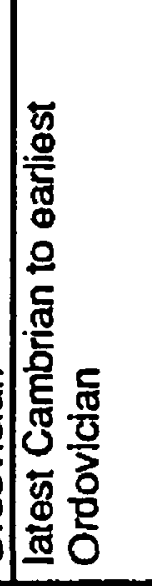 & 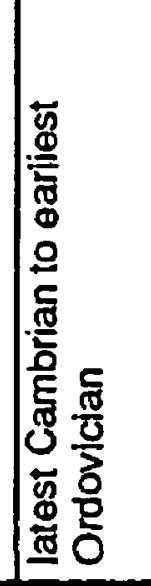 & 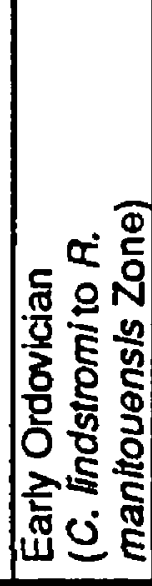 & 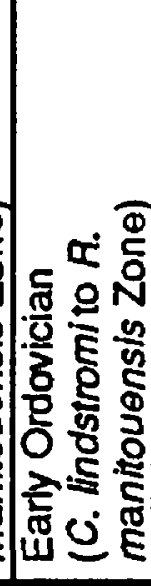 & 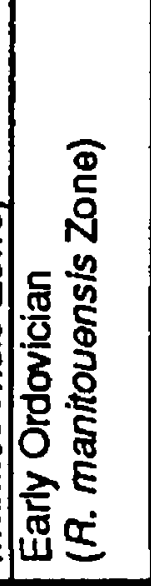 & 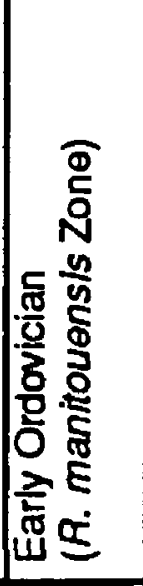 & 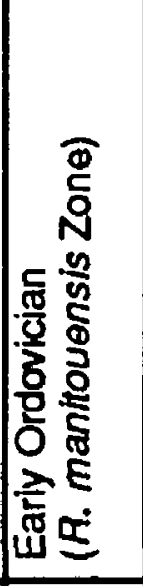 & 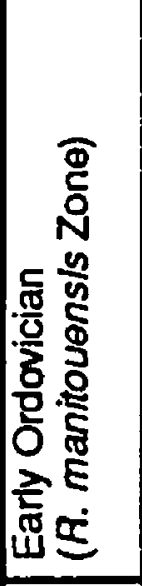 & 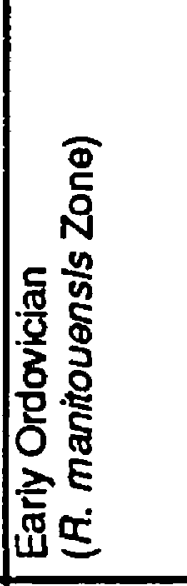 & 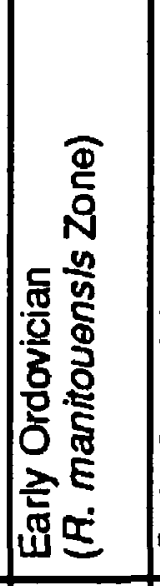 & 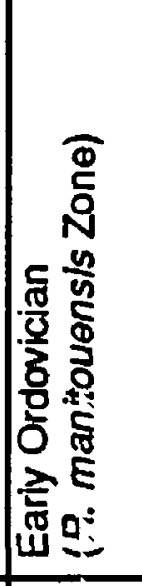 & 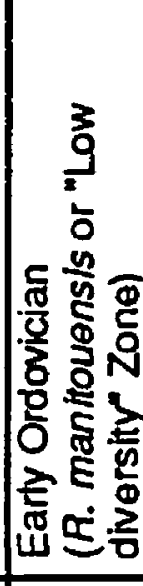 & 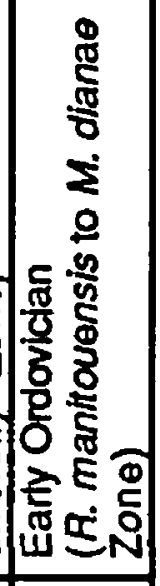 \\
\hline 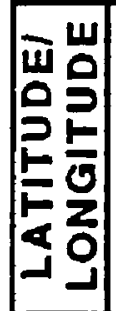 & 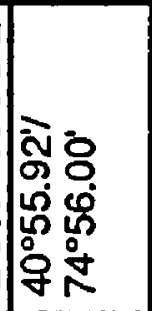 & 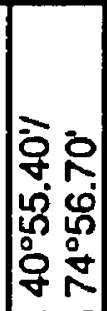 & 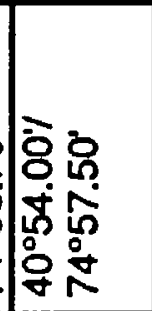 & 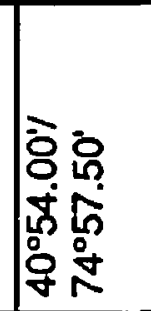 & 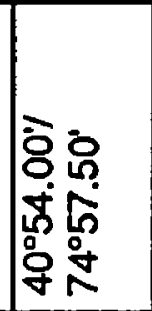 & 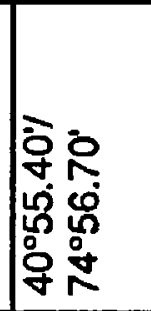 & 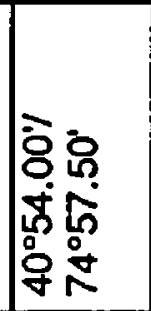 & 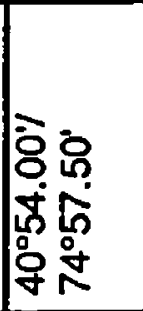 & 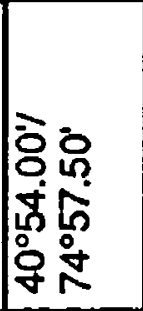 & 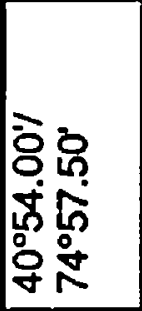 & 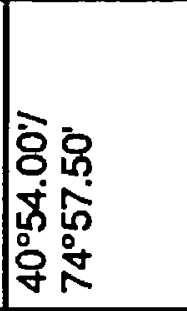 & 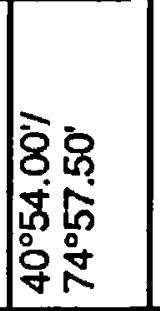 & 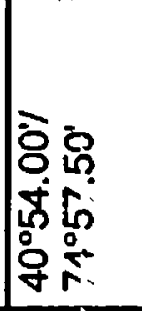 & 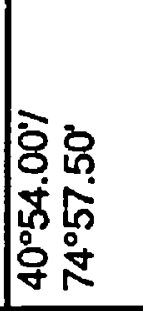 & 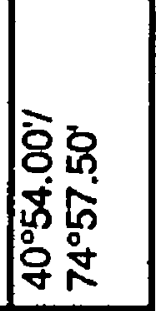 \\
\hline $\mid \begin{array}{l}z \\
z \\
z \\
0 \\
0 \\
0\end{array}$ & 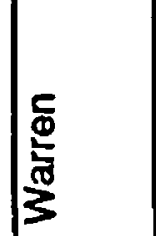 & 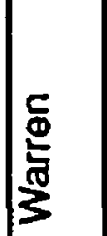 & 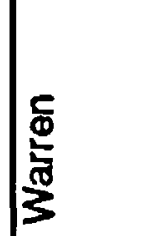 & 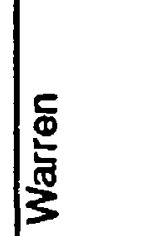 & 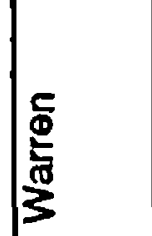 & 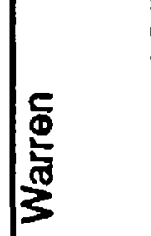 & $\mid \frac{c}{\Phi}$ & 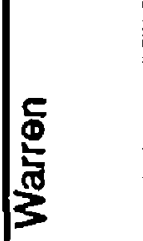 & 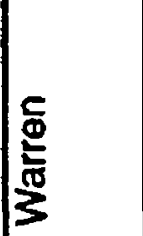 & 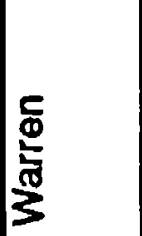 & 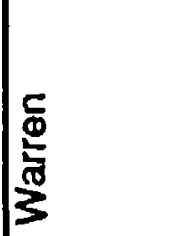 & $\mid \frac{c}{\Phi}$ & 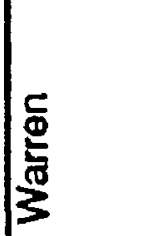 & 点 & 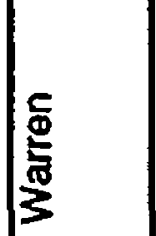 \\
\hline 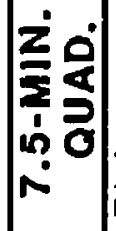 & $\frac{5}{\frac{5}{0}}$ & $\frac{5}{\frac{5}{0}}$ & 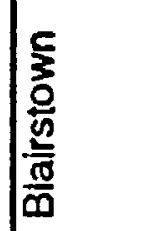 & 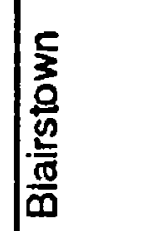 & 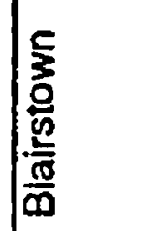 & 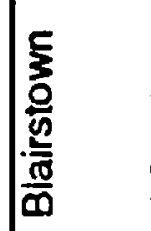 & $\frac{5}{\frac{5}{0}}$ & 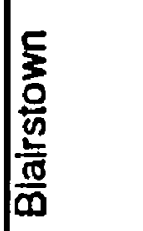 & $\frac{5}{\frac{5}{0}}$ & 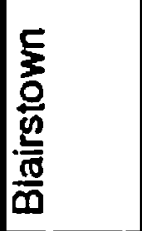 & 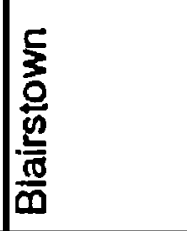 & 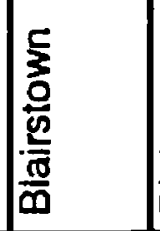 & 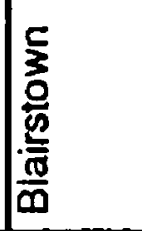 & 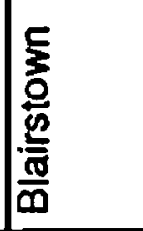 & 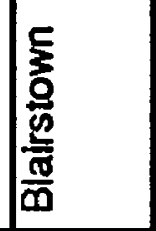 \\
\hline $\mid \begin{array}{ll}0 & 0 \\
z & z \\
0 & 0 \\
1 & 0 \\
\frac{w}{4} & 0\end{array}$ & 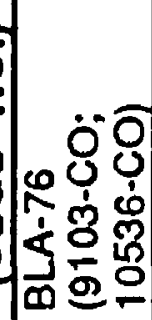 & 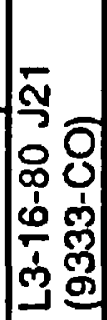 & 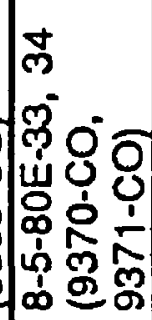 & 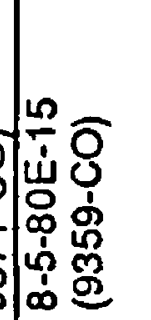 & 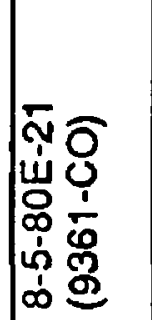 & 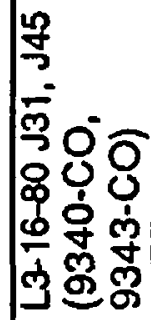 & 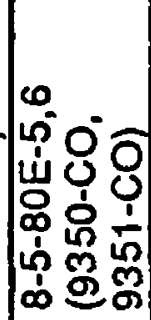 & 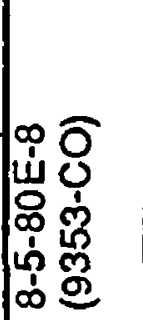 & 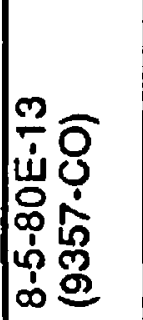 & 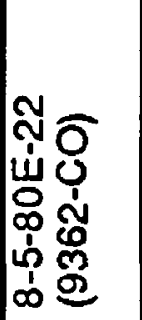 & 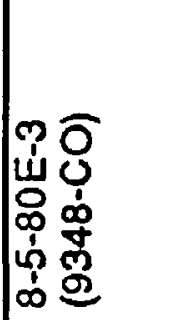 & 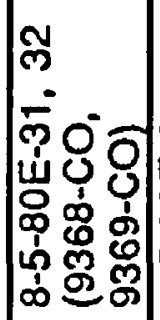 & 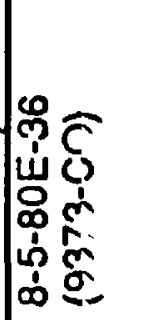 & 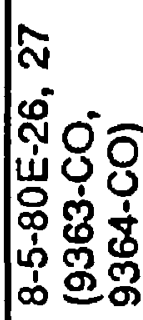 & 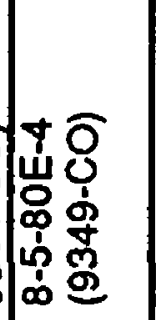 \\
\hline
\end{tabular}




\begin{tabular}{|c|c|c|c|c|c|c|c|c|c|c|c|c|c|c|c|c|}
\hline 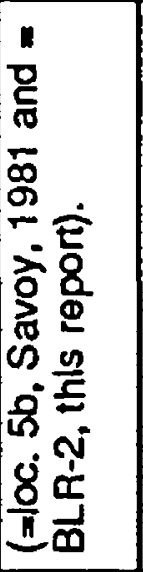 & 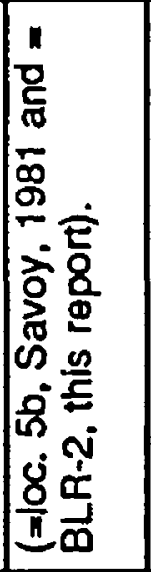 & 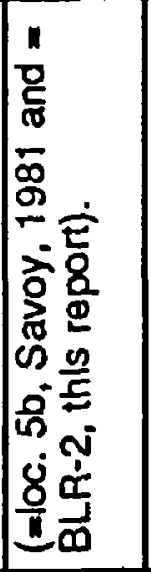 & 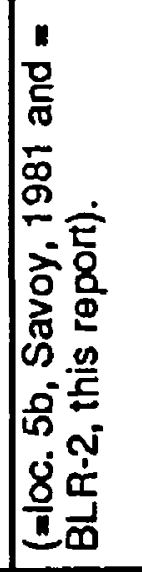 & 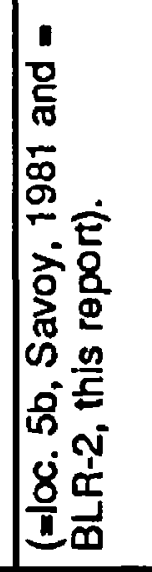 & 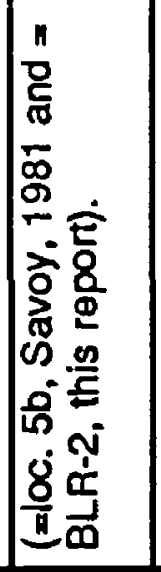 & 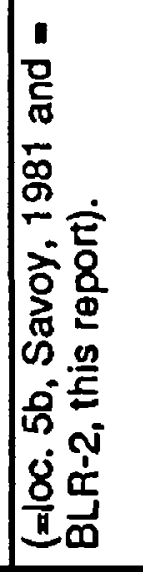 & 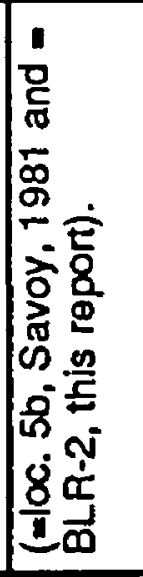 & 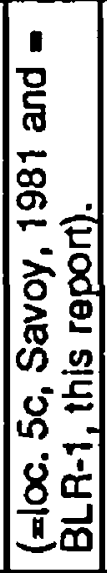 & 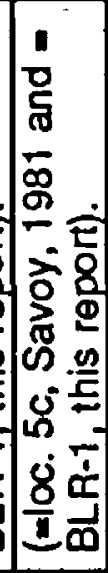 & 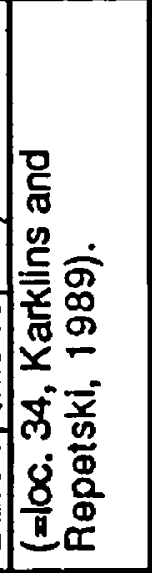 & 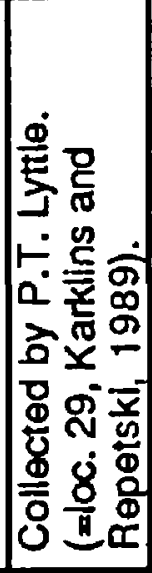 & 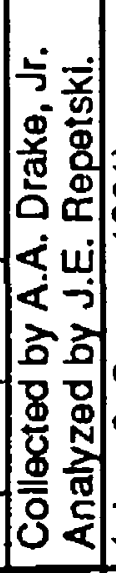 & 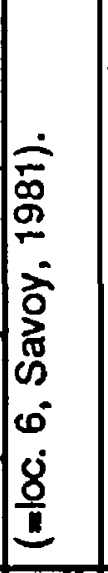 & 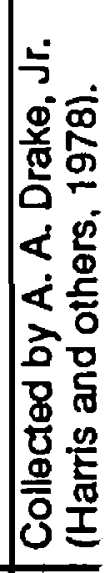 & 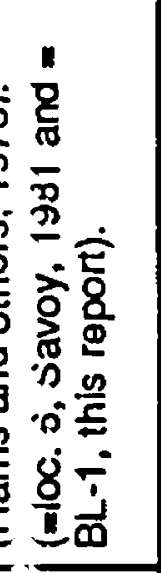 & 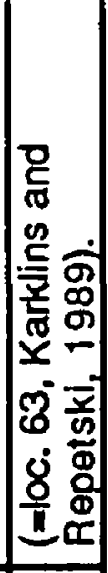 \\
\hline in & in & in & | & | & | & م & in & | & in & מת & מא & $\mid \begin{array}{l}n \\
0 \\
0 \\
0 \\
0\end{array}$ & 100 & on & $n$ & \\
\hline 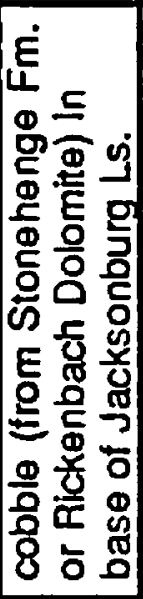 & 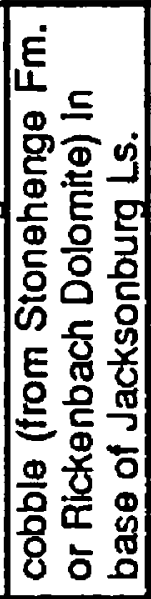 & 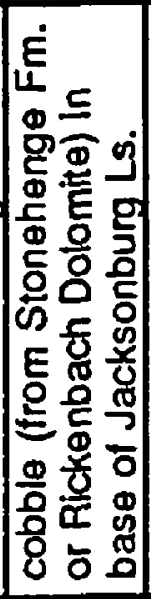 & 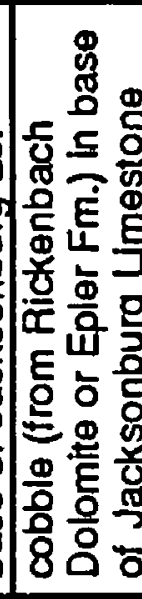 & 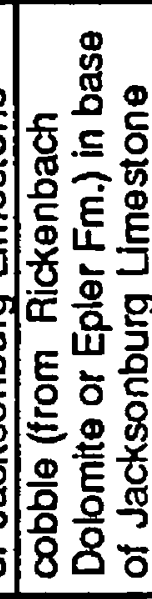 & 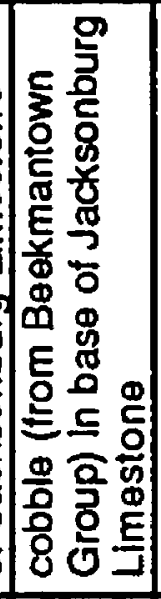 & 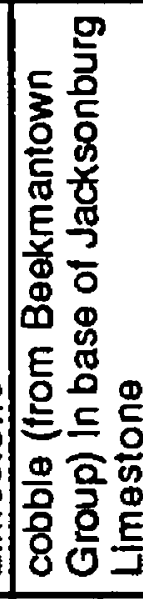 & 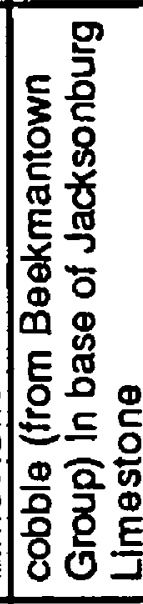 & 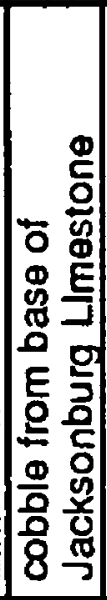 & 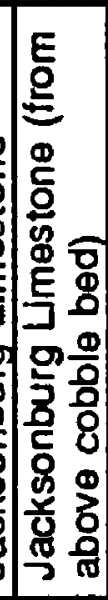 & 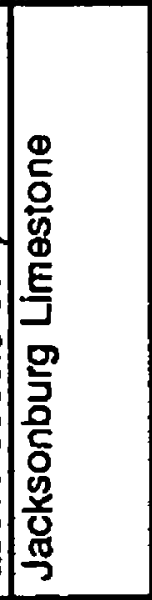 & 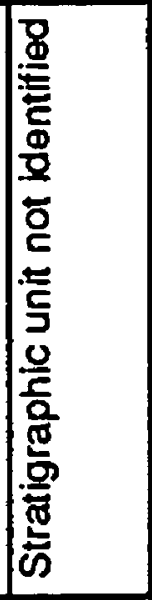 & 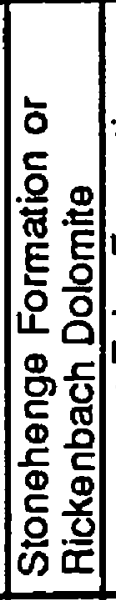 & 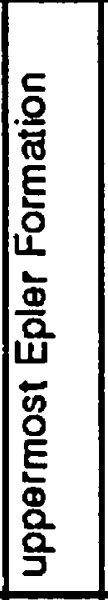 & 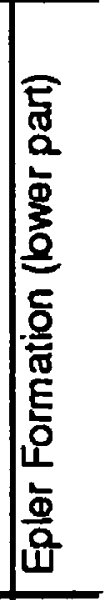 & 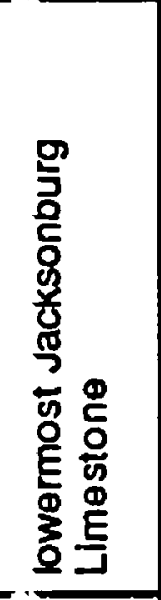 & 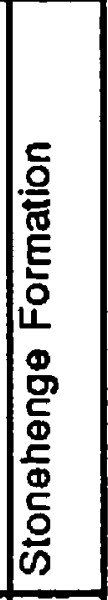 \\
\hline 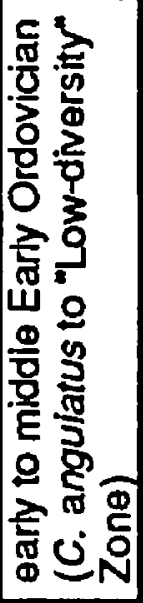 & 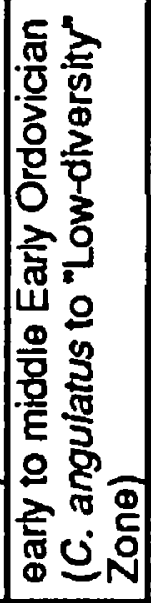 & 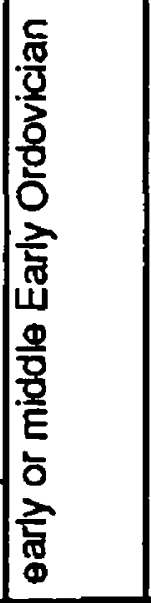 & 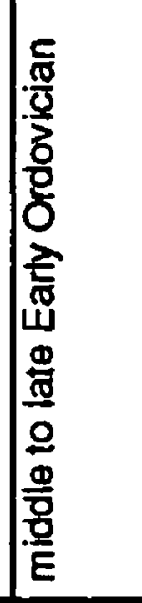 & 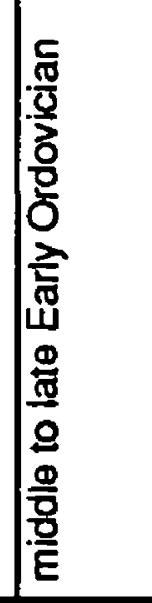 & 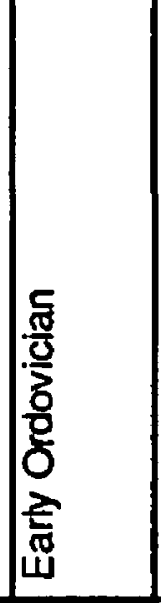 & 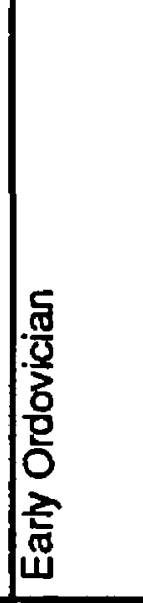 & 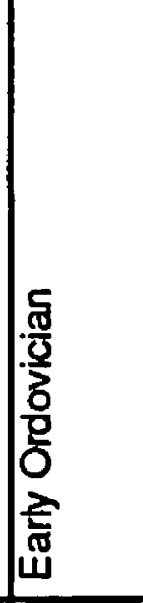 & 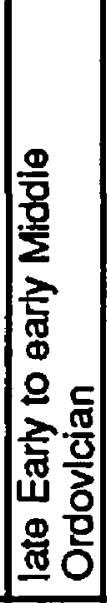 & 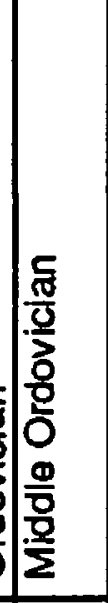 & 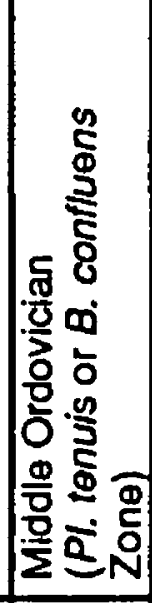 & 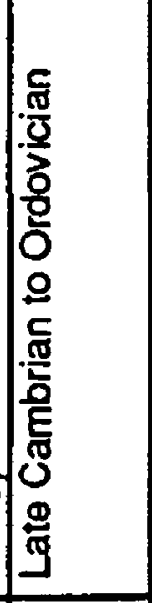 & 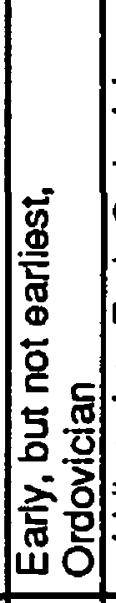 & 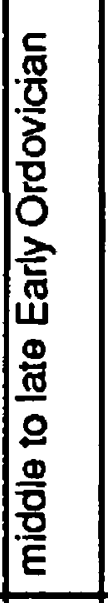 & 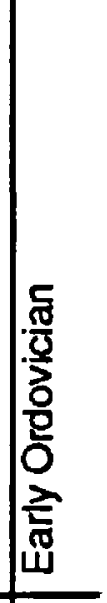 & 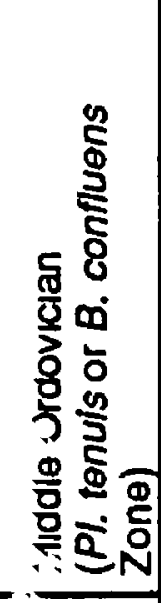 & 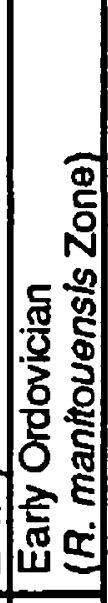 \\
\hline 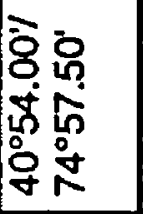 & 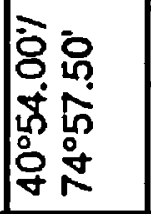 & 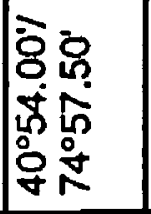 & 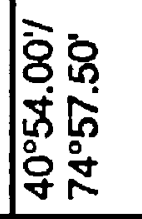 & 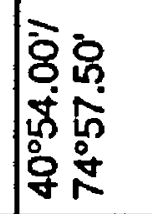 & 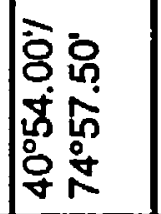 & 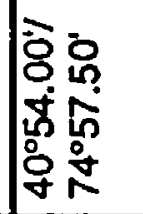 & 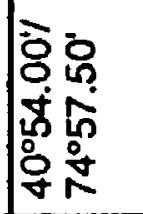 & 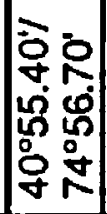 & 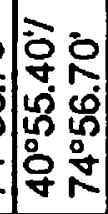 & 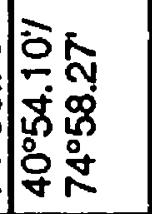 & 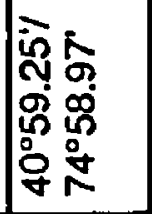 & 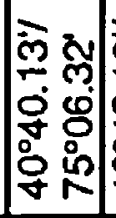 & 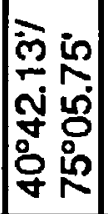 & 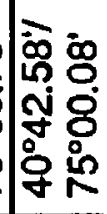 & 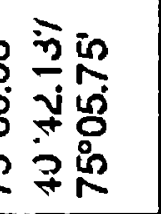 & 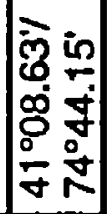 \\
\hline 点 & 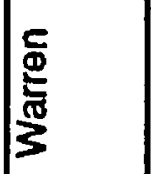 & 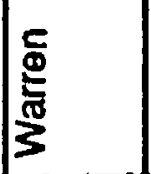 & 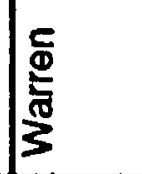 & 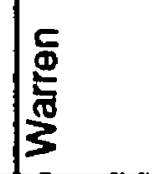 & 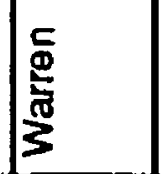 & 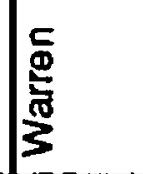 & 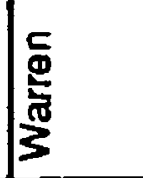 & 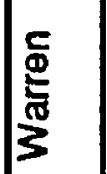 & 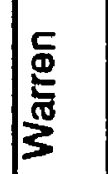 & $\mid \begin{array}{l}\frac{5}{\Phi} \\
\frac{2}{\pi / 2} \\
3\end{array}$ & 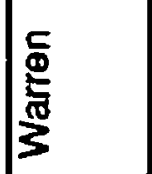 & 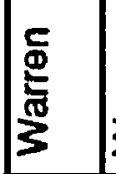 & 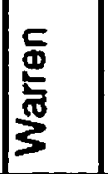 & 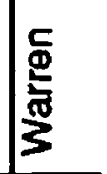 & & 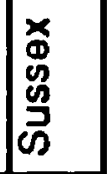 \\
\hline 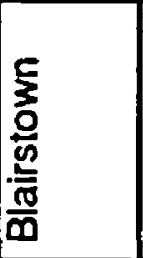 & 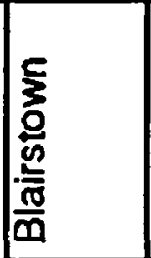 & 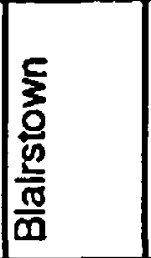 & 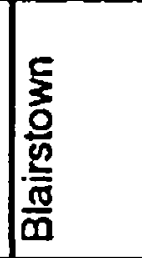 & 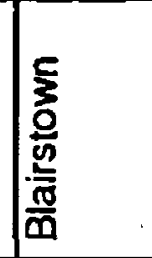 & 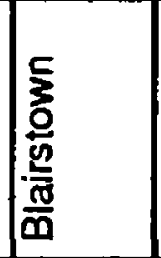 & $\frac{5}{\frac{5}{0}}$ & $\begin{array}{l}\frac{5}{3} \\
\frac{0}{20} \\
\frac{\frac{5}{0}}{\overline{0}} \\
\frac{0}{0}\end{array}$ & 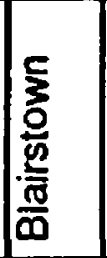 & 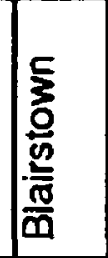 & 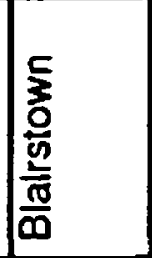 & 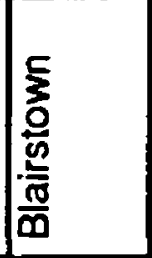 & $\mid \begin{array}{l}z \\
5 \\
0 \\
0 \\
\underline{0} \\
0 \\
0 \\
0\end{array}$ & 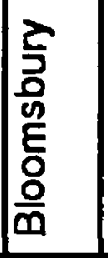 & 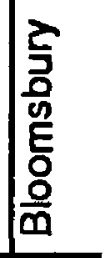 & 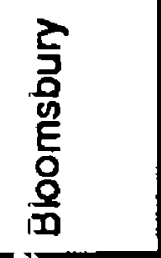 & 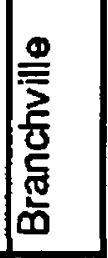 \\
\hline 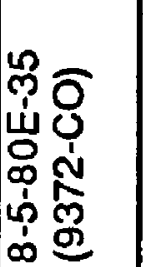 & 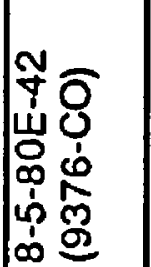 & 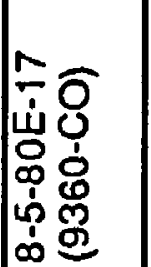 & 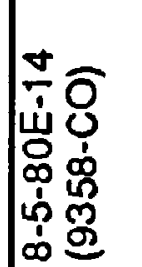 & 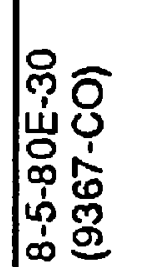 & 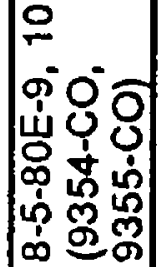 & 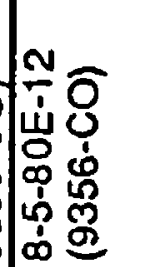 & 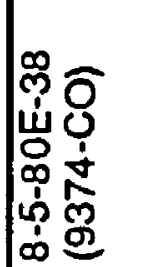 & 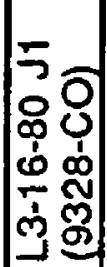 & 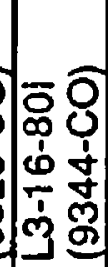 & 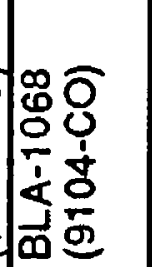 & 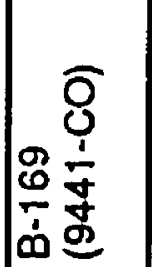 & 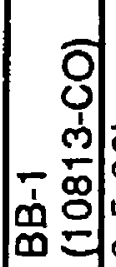 & 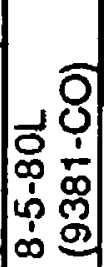 & 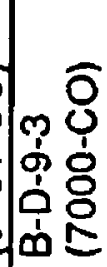 & 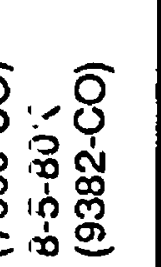 & 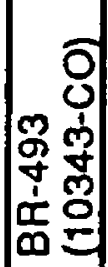 \\
\hline
\end{tabular}




\begin{tabular}{|c|c|c|c|c|c|c|c|c|c|c|c|c|c|c|c|c|}
\hline 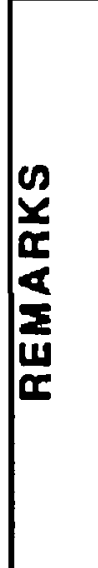 & 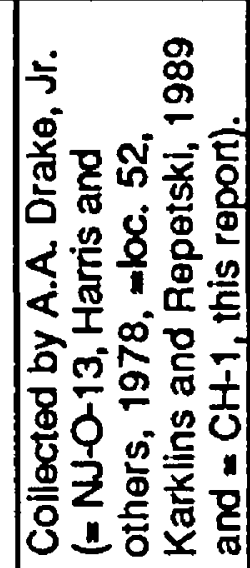 & 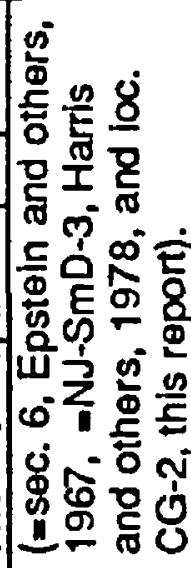 & 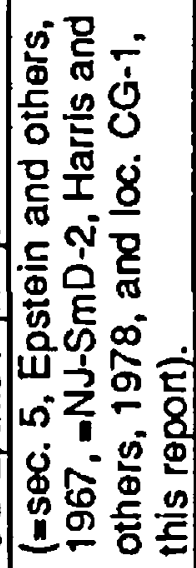 & 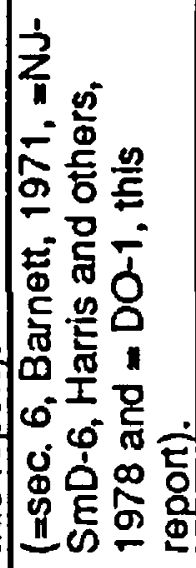 & 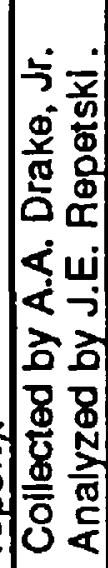 & 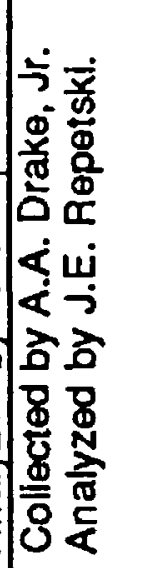 & 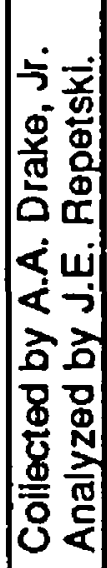 & 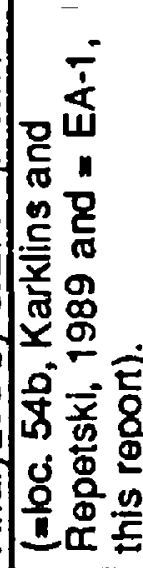 & 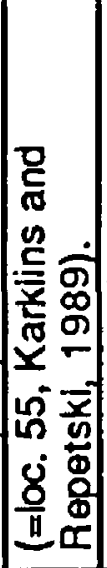 & 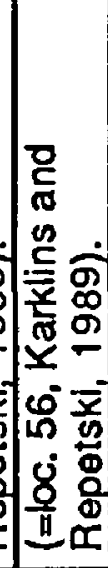 & 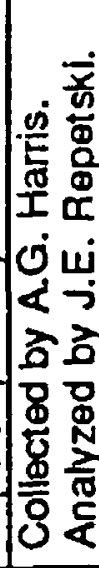 & 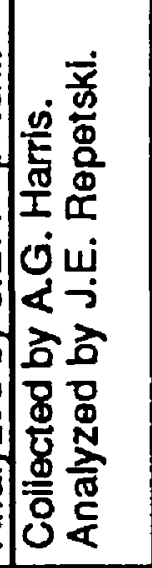 & 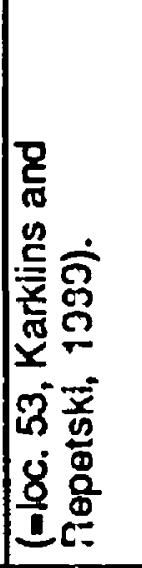 & 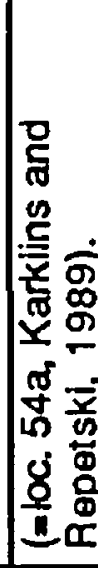 & 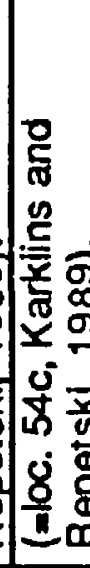 & 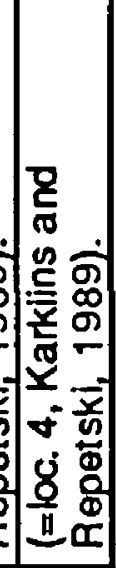 \\
\hline $\mid \bar{s}$ & 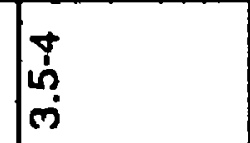 & مـ & مسا & $\begin{array}{l}n \\
0 \\
0 \\
+ \\
0\end{array}$ & $\begin{array}{l}n \\
0 \\
0 \\
10 \\
0\end{array}$ & 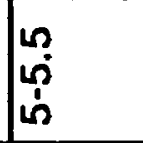 & 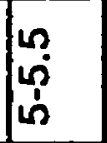 & | & 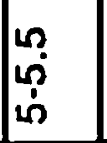 & 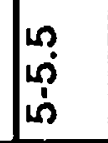 & in & L & 100 & مـ & م/ & م \\
\hline 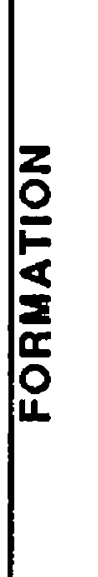 & 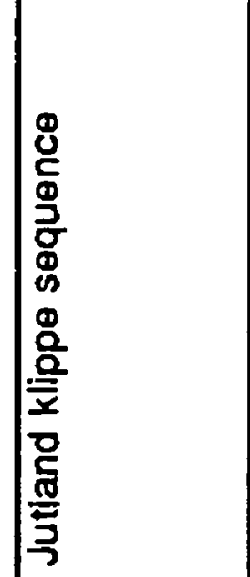 & 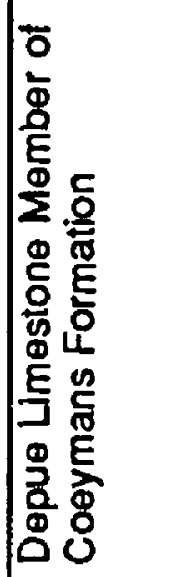 & 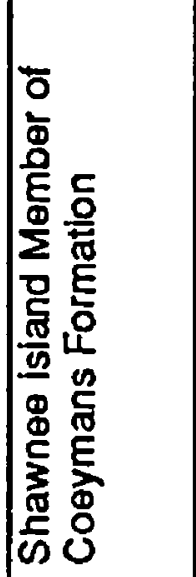 & 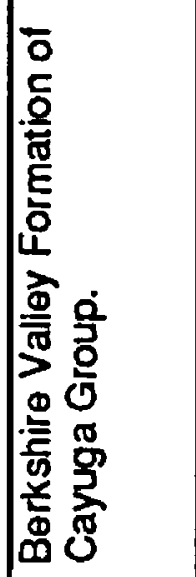 & 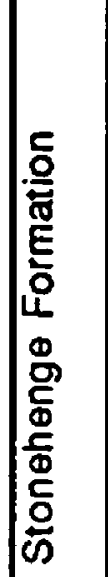 & 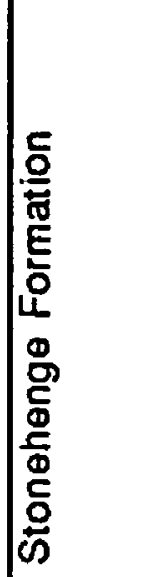 & 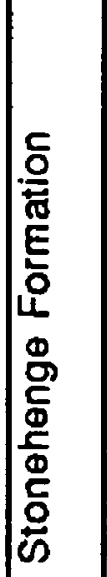 & 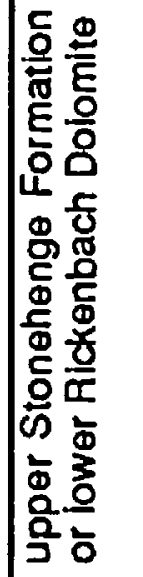 & 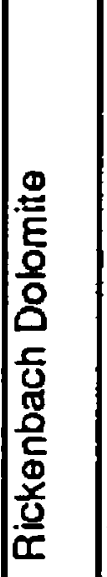 & 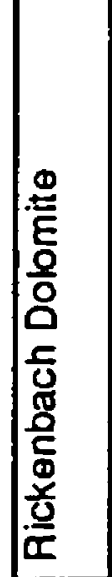 & 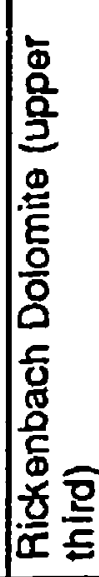 & 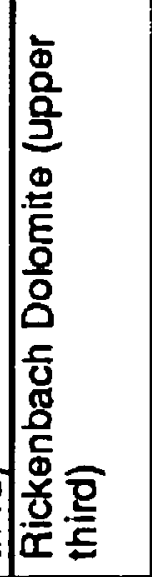 & 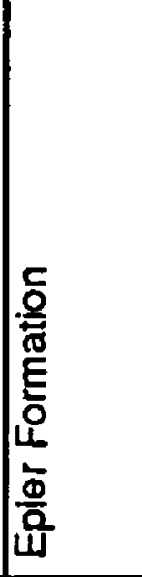 & 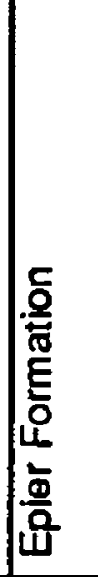 & 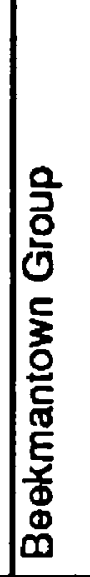 & 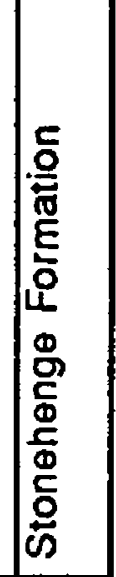 \\
\hline 岁 & 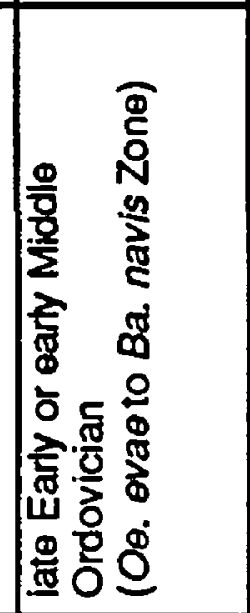 & 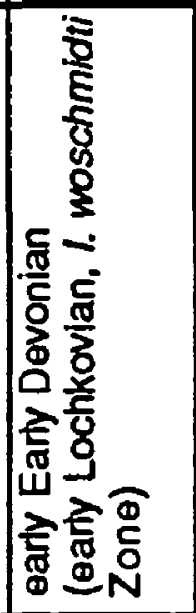 & 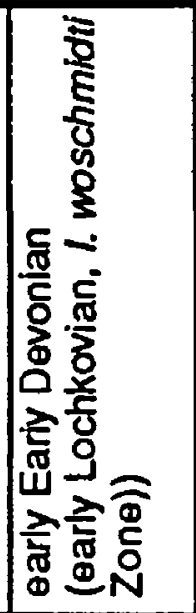 & 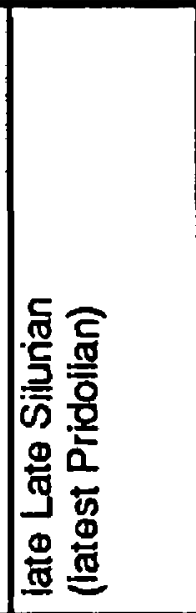 & 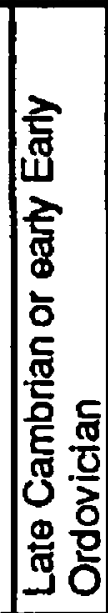 & 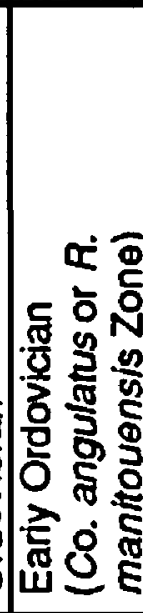 & 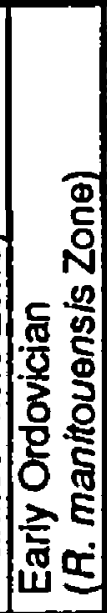 & 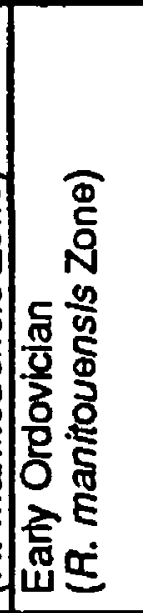 & 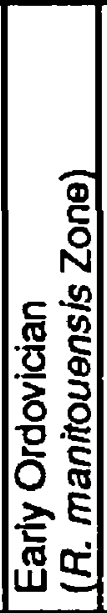 & 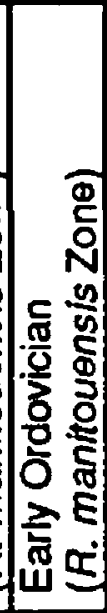 & 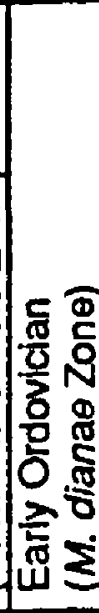 & 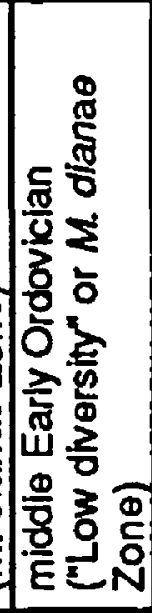 & 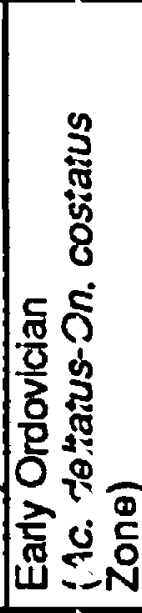 & 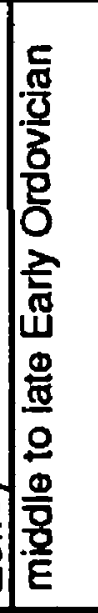 & $\mid \begin{array}{l}\frac{5}{0} \\
\frac{\pi}{0} \\
\frac{0}{3} \\
0 \\
0\end{array}$ & 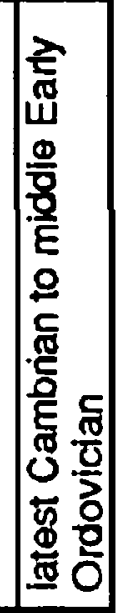 \\
\hline 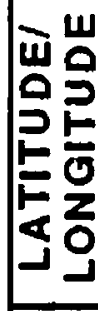 & 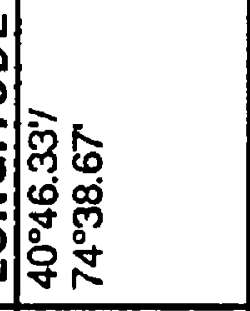 & 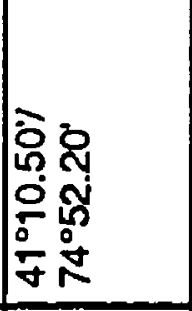 & 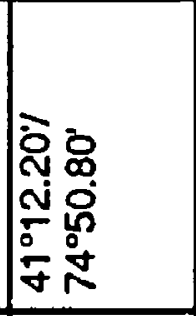 & 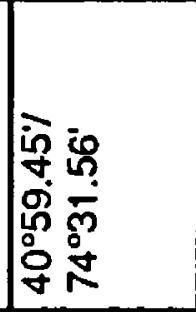 & 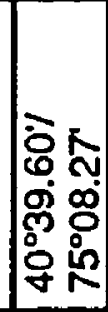 & 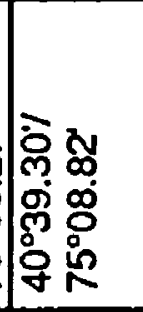 & 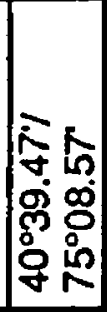 & 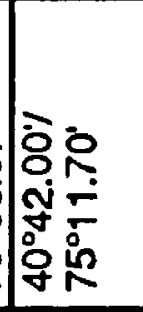 & 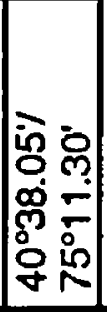 & 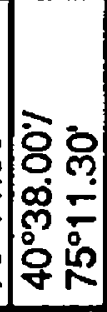 & 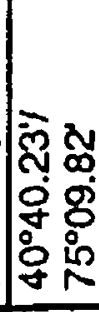 & 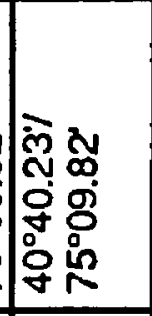 & 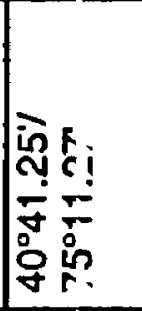 & 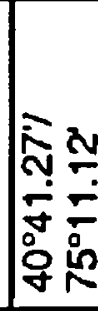 & 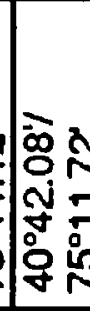 & \\
\hline $\begin{array}{l}\mathbf{E} \\
\\
\\
0 \\
0\end{array}$ & $\frac{a}{\frac{9}{2}}$ & 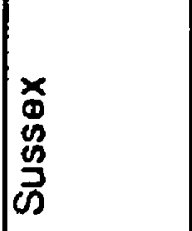 & 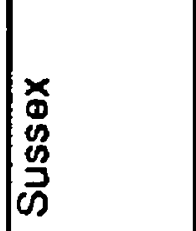 & $\frac{\text { 은 }}{\frac{2}{2}}$ & 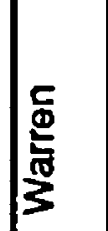 & 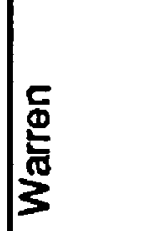 & 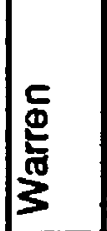 & 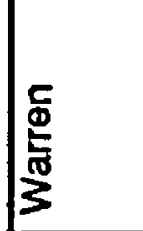 & 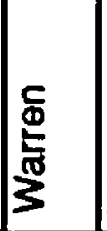 & 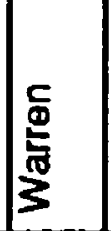 & 点 & 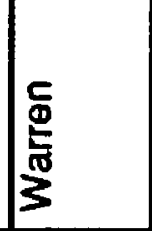 & 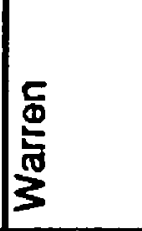 & 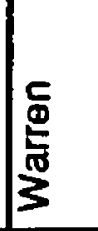 & 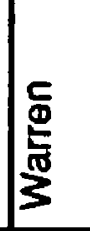 & 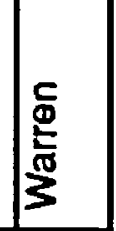 \\
\hline 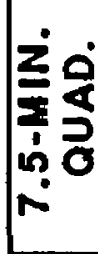 & 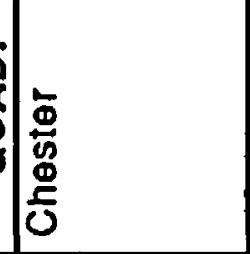 & 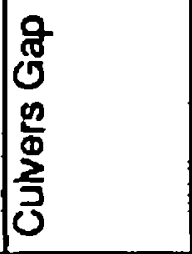 & 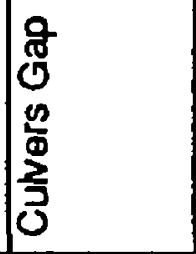 & 产 & 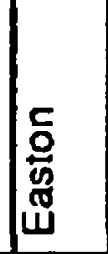 & 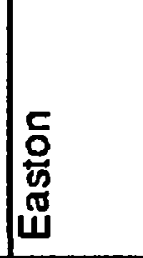 & 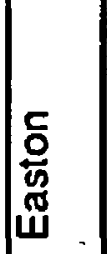 & 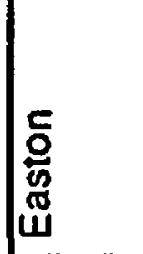 & 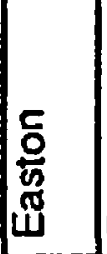 & 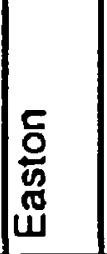 & 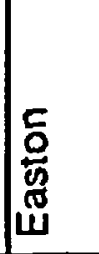 & 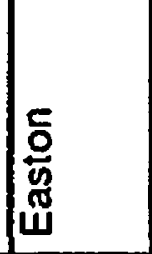 & 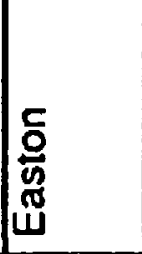 & 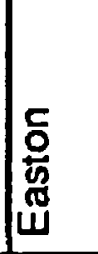 & 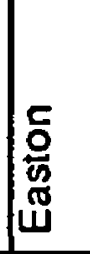 & $\begin{array}{l}8 \\
\frac{2}{0} \\
\frac{7}{6} \\
\frac{\pi}{6}\end{array}$ \\
\hline 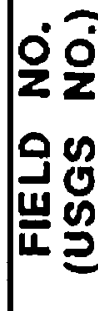 & 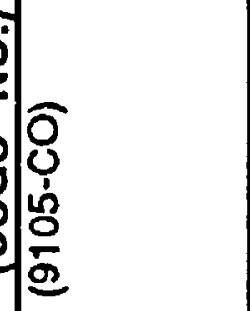 & 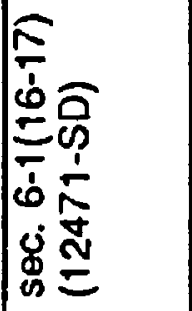 & 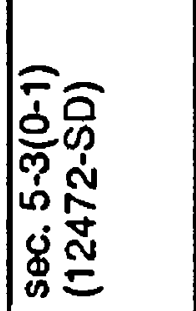 & $\begin{array}{l}0 \\
0\end{array}$ & 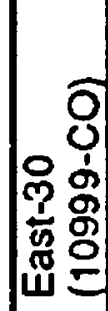 & 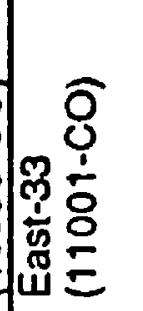 & & 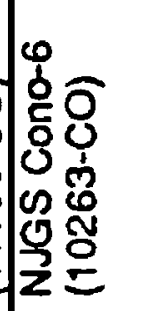 & 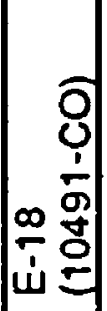 & 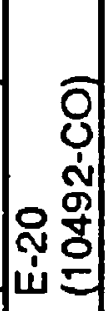 & $\begin{array}{l}0 \\
0 \\
0 \\
8 \\
0\end{array}$ & 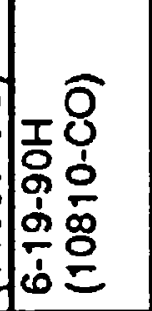 & 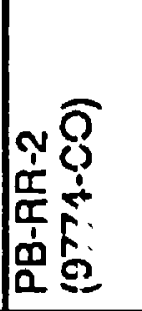 & 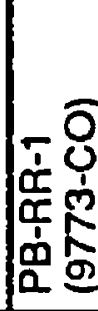 & o & 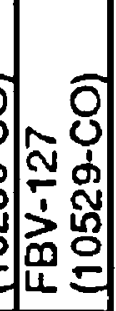 \\
\hline
\end{tabular}




\begin{tabular}{|c|c|c|c|c|c|c|c|c|c|c|c|c|c|c|c|c|c|}
\hline 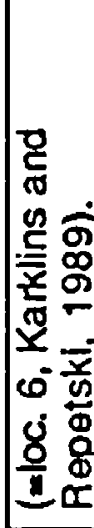 & 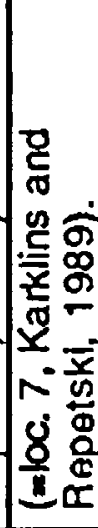 & 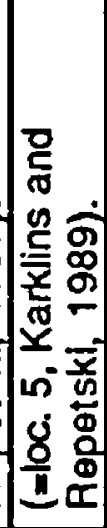 & 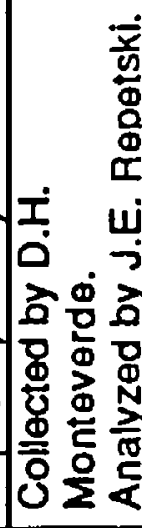 & 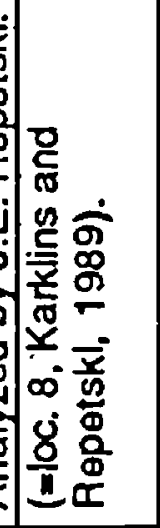 & 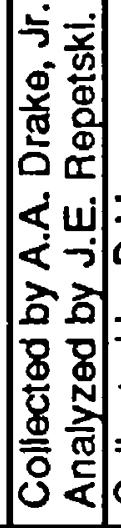 & 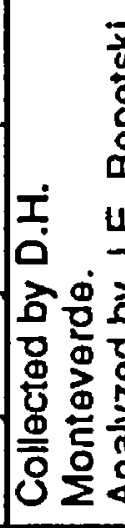 & 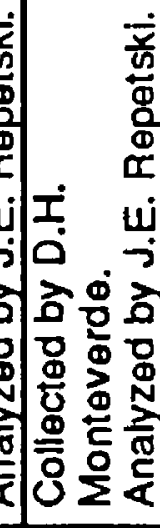 & 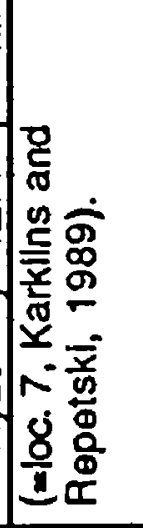 & 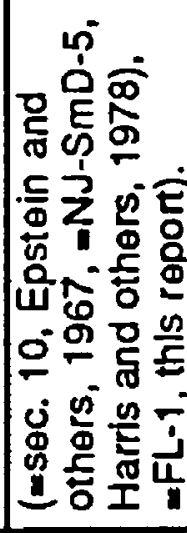 & 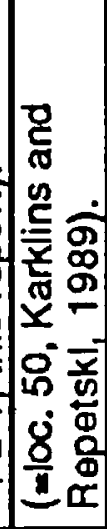 & 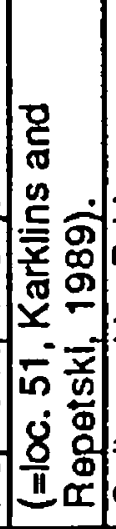 & 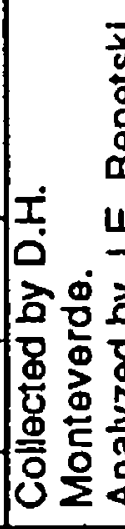 & 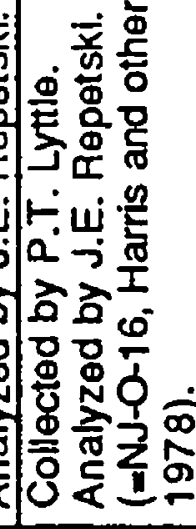 & 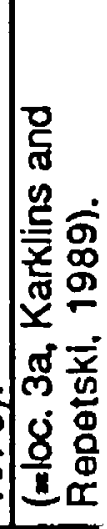 & 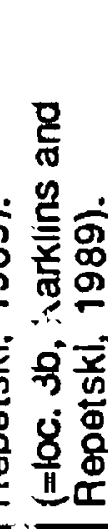 & 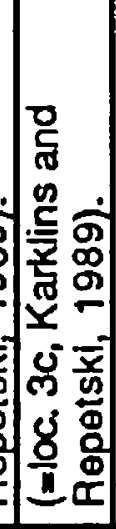 & 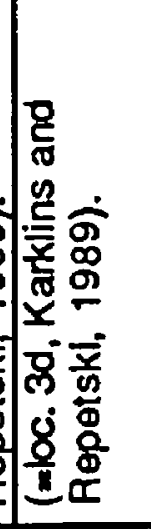 \\
\hline$\Omega$ & in & in & in & مו & in & L & م & 10 & 10 & 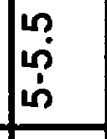 & in & 10 & 10 & In & I & 100 & L \\
\hline 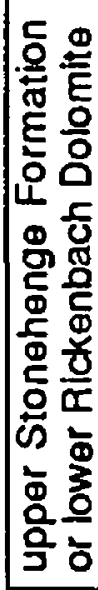 & 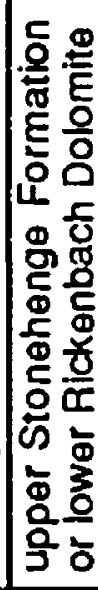 & 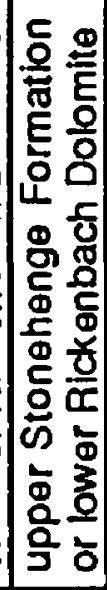 & 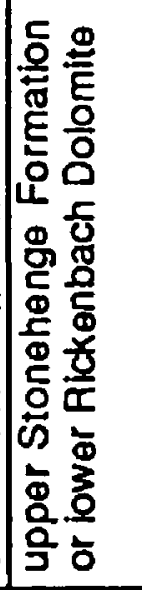 & 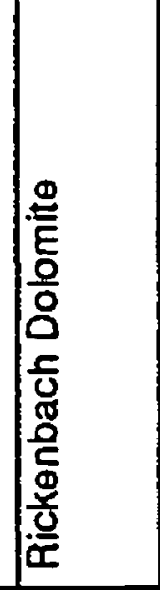 & 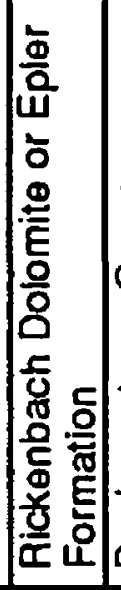 & 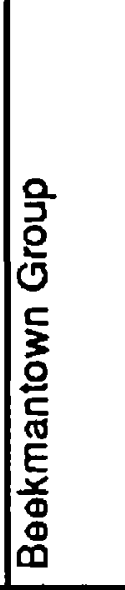 & 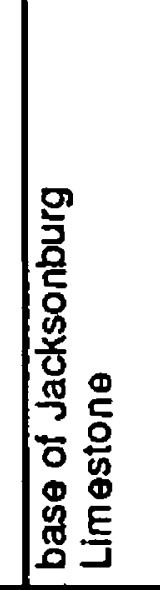 & 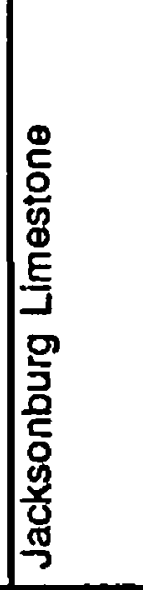 & 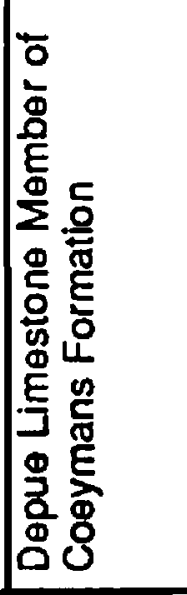 & 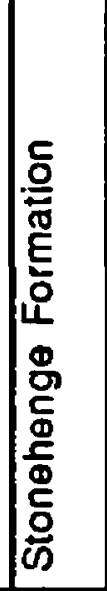 & 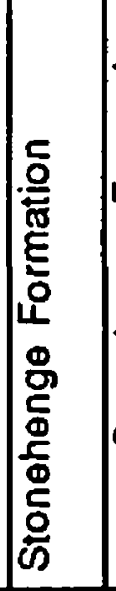 & 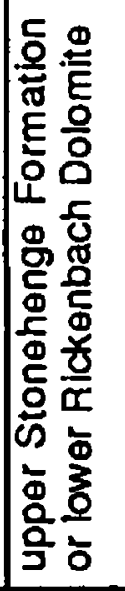 & 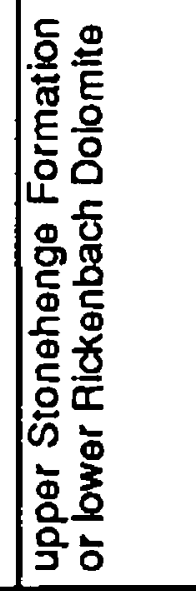 & 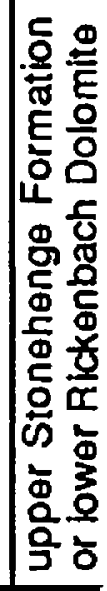 & 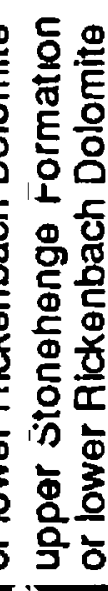 & 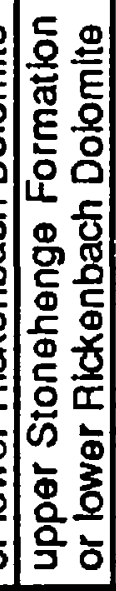 & 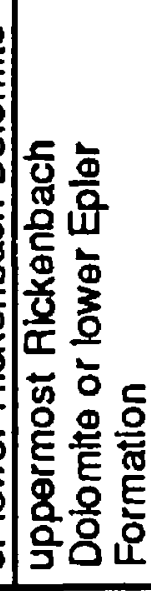 \\
\hline 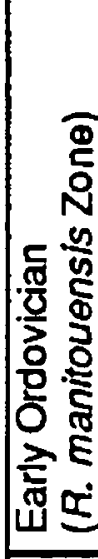 & 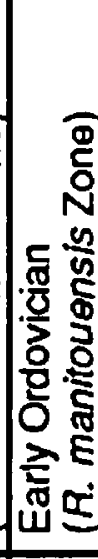 & 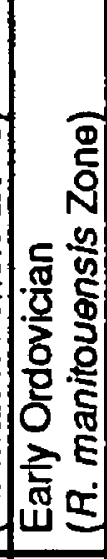 & 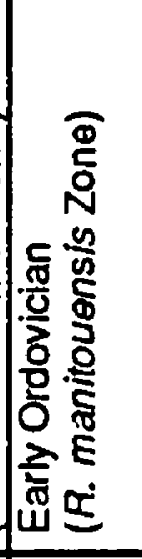 & 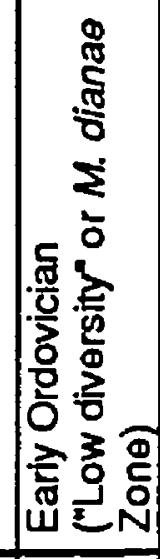 & 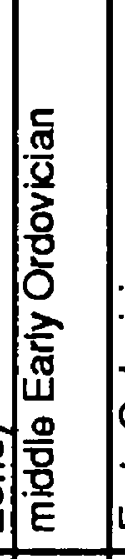 & 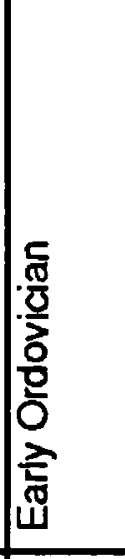 & 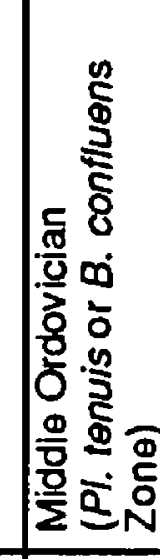 & 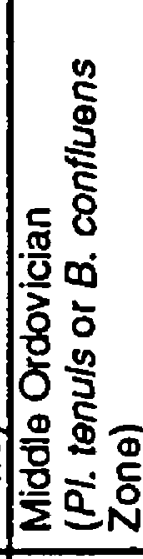 & 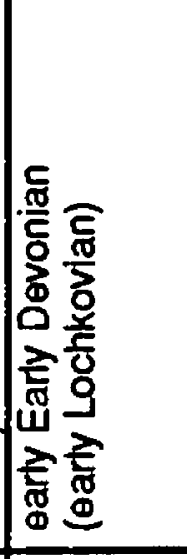 & 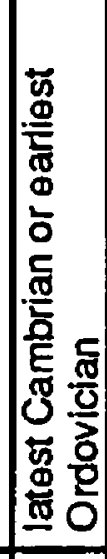 & 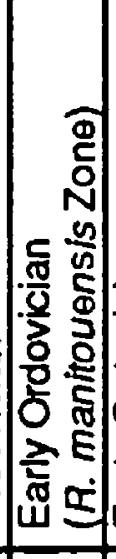 & 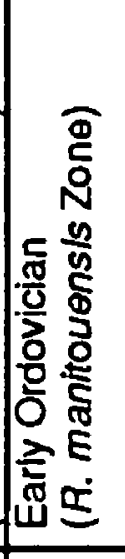 & 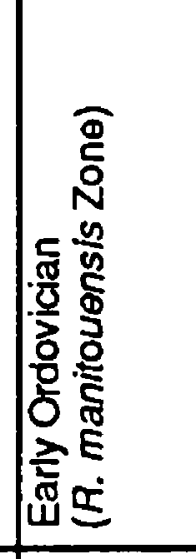 & 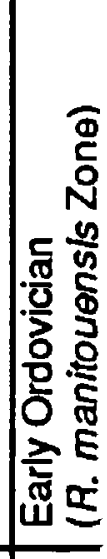 & 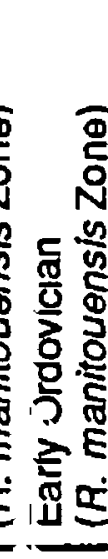 & 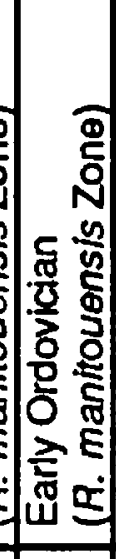 & 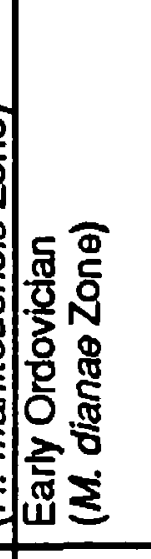 \\
\hline 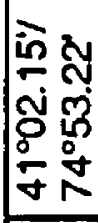 & 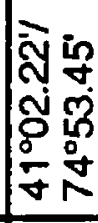 & 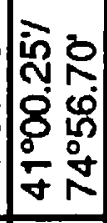 & 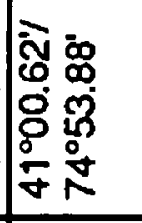 & 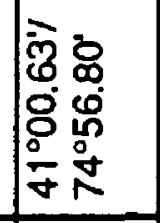 & 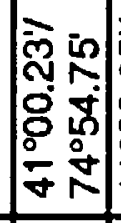 & 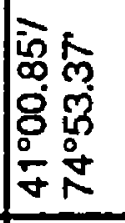 & 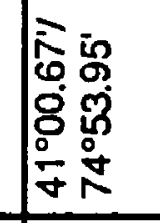 & 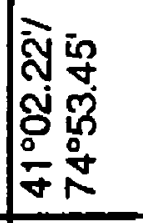 & 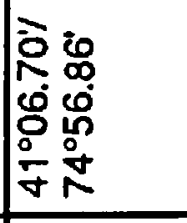 & 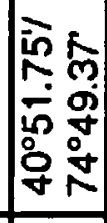 & 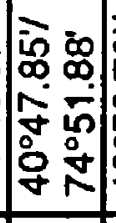 & 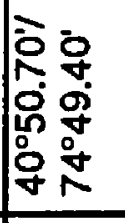 & 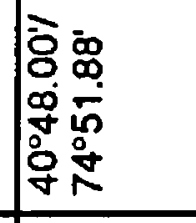 & 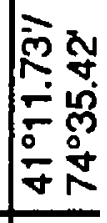 & 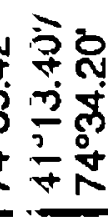 & 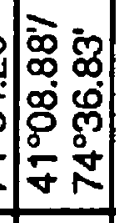 & 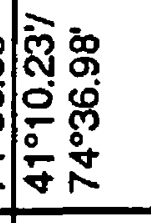 \\
\hline 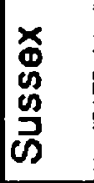 & 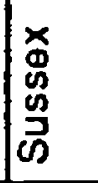 & 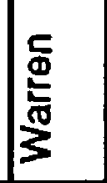 & 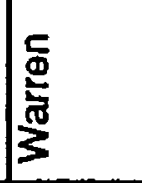 & 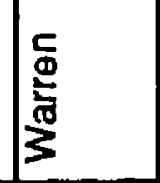 & 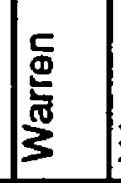 & 点 & 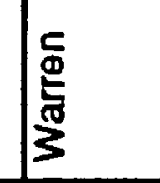 & 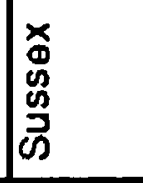 & 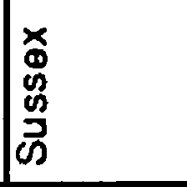 & 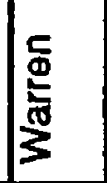 & 缹 & $\frac{9}{\frac{0}{2}}$ & $\mid \frac{9}{5}$ & 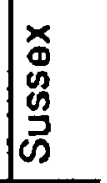 & 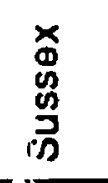 & 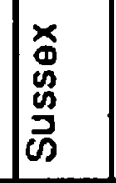 & $\begin{array}{l}x \\
0 \\
0 \\
0 \\
\tilde{n} \\
\tilde{n}\end{array}$ \\
\hline $\begin{array}{l}\frac{\gamma}{8} \\
\frac{0}{0} \\
\frac{0}{0} \\
\frac{0}{4}\end{array}$ & 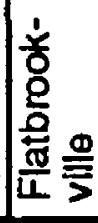 & 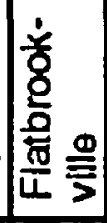 & 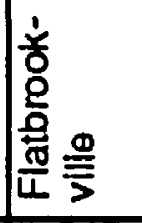 & 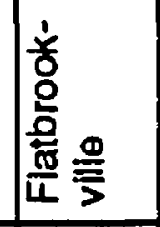 & 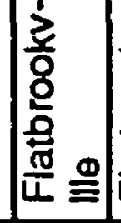 & 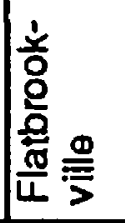 & 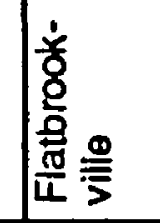 & 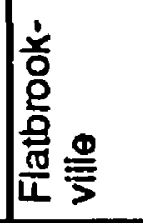 & 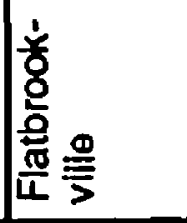 & 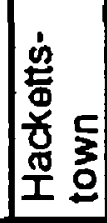 & 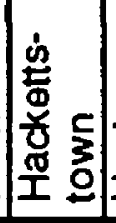 & 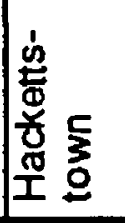 & 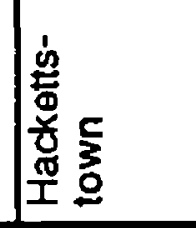 & 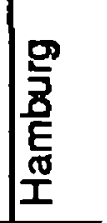 & 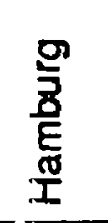 & 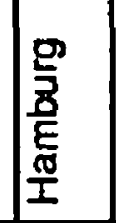 & 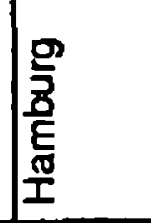 \\
\hline 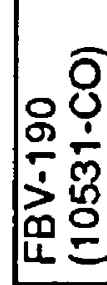 & 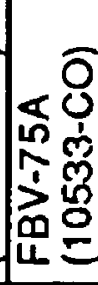 & 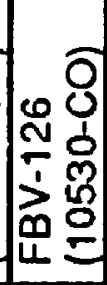 & 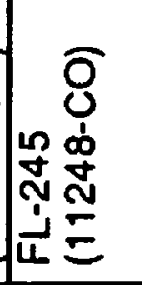 & 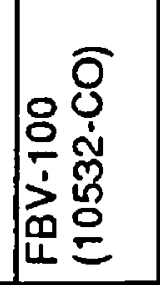 & 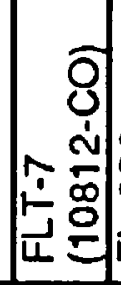 & 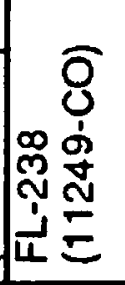 & 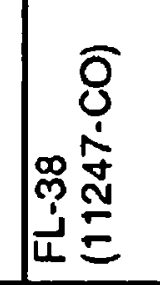 & 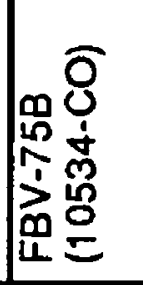 & 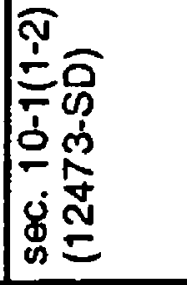 & 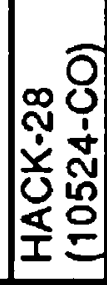 & 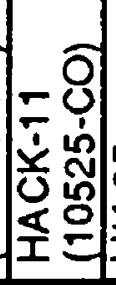 & 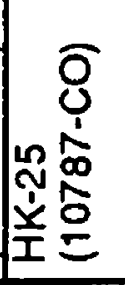 & 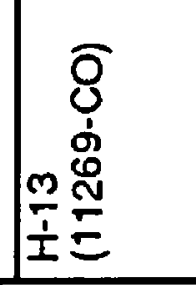 & 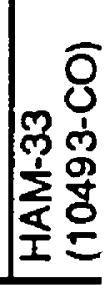 & 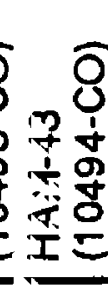 & 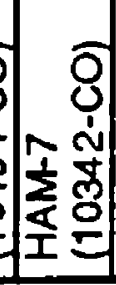 & 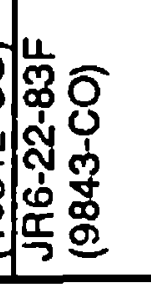 \\
\hline
\end{tabular}




\begin{tabular}{|c|c|c|c|c|c|c|c|c|c|c|c|c|c|c|c|c|c|c|c|c|}
\hline & 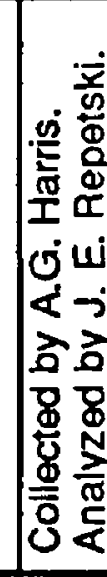 & 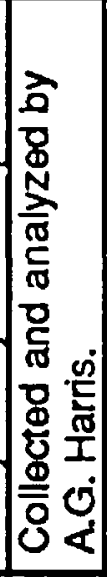 & 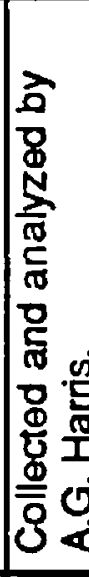 & 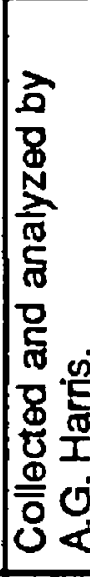 & 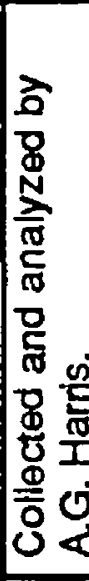 & 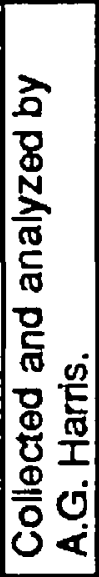 & 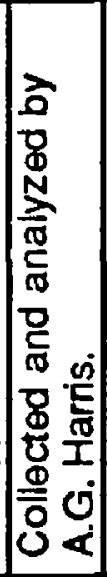 & 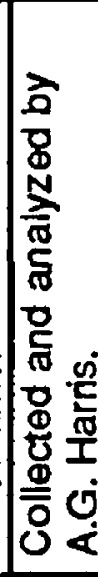 & 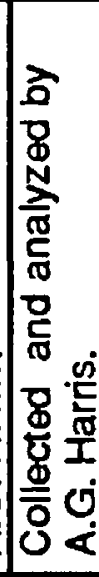 & 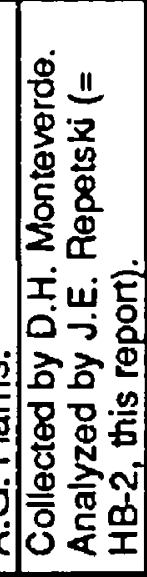 & 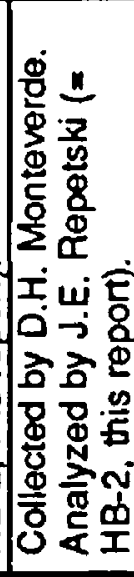 & 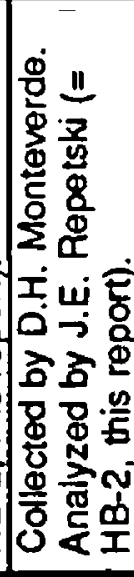 & 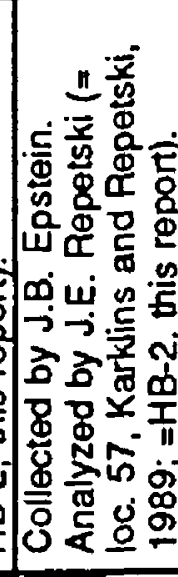 & 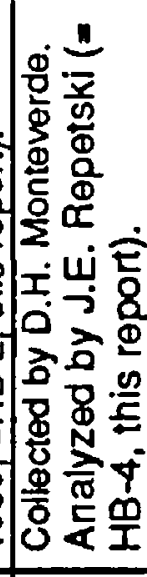 & 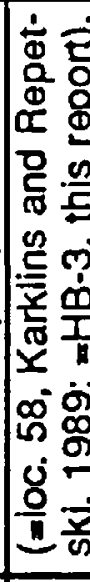 & 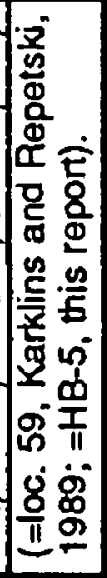 & 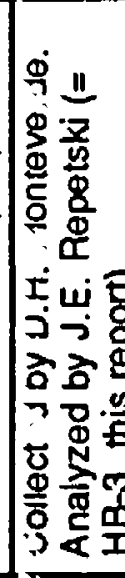 & 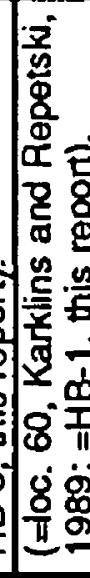 & 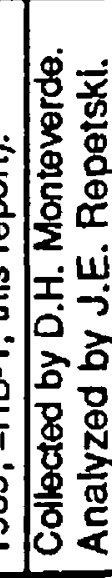 & 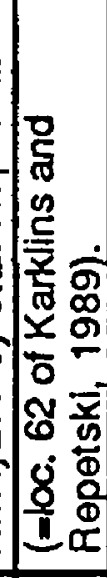 \\
\hline & م & |n & in & 10 & |n & مسا & in & م/ & م & $\begin{array}{l}8 \\
2\end{array}$ & & $\lambda^{\prime}$ & $\stackrel{\infty}{\circ}$ & $\forall$ & $\mid \begin{array}{l}n \\
\dot{y} \\
y\end{array}$ & 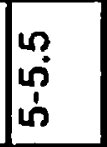 & $\stackrel{?}{+}$ & $\stackrel{n}{\dot{f}}$ & $\forall$ & 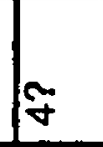 \\
\hline & 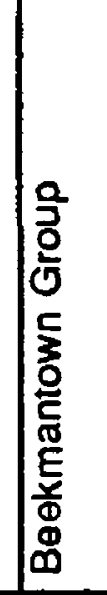 & 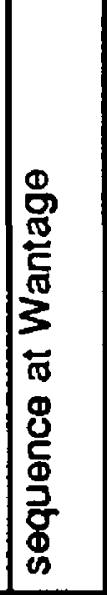 & 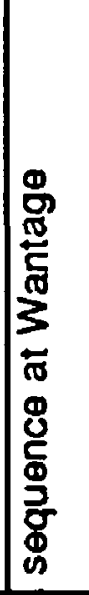 & 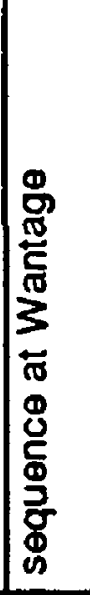 & 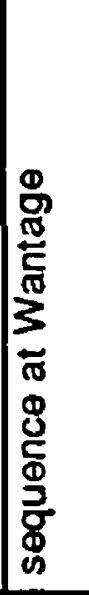 & 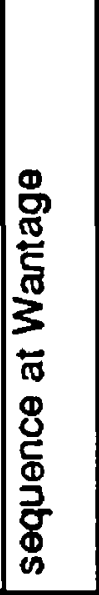 & 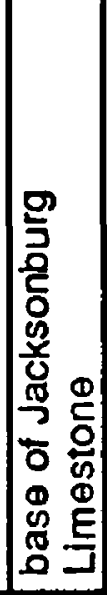 & 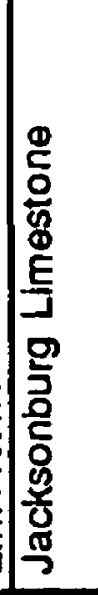 & 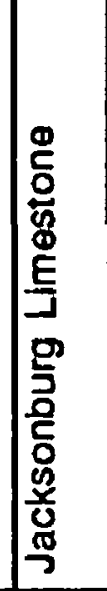 & 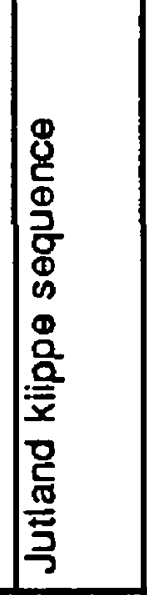 & 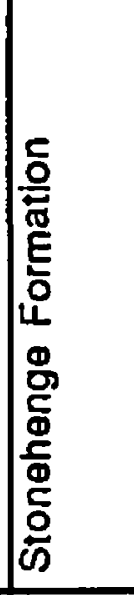 & 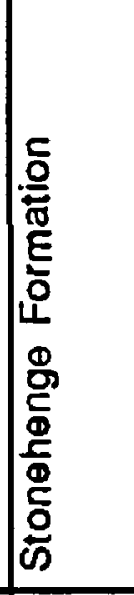 & 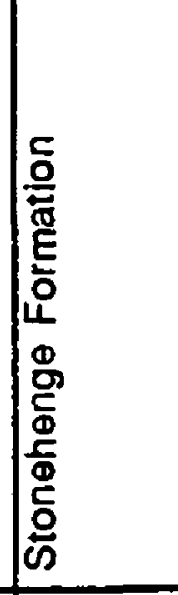 & 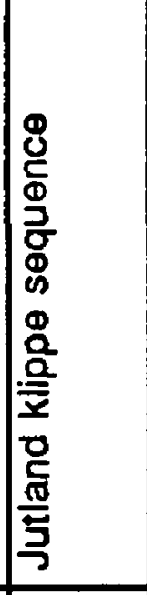 & 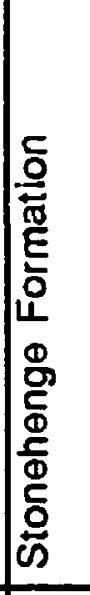 & 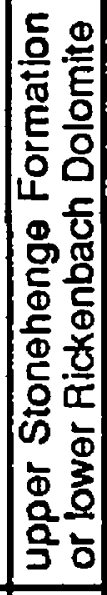 & 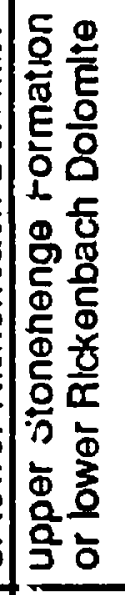 & 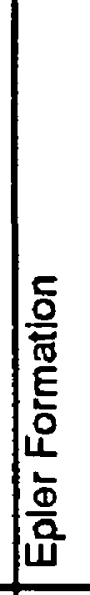 & 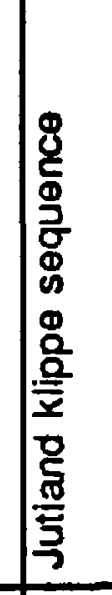 & 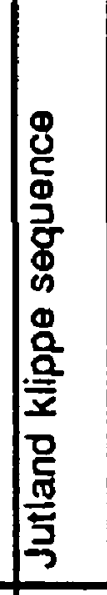 \\
\hline
\end{tabular}

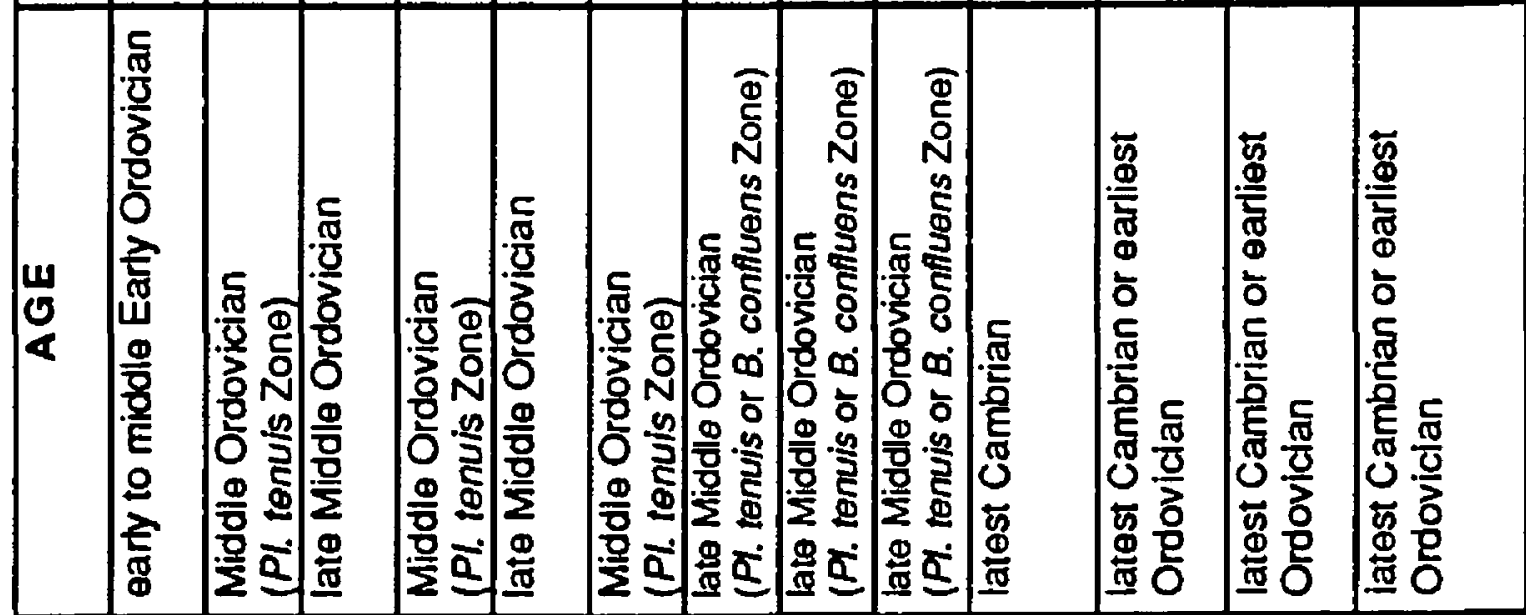

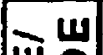

눙을

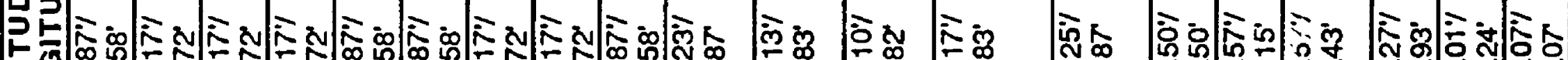
Ez

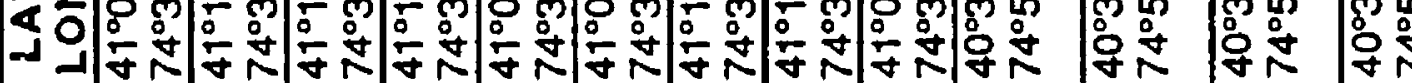

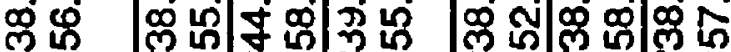

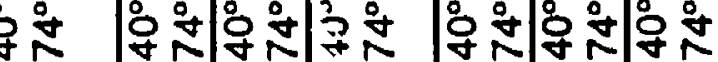

\begin{tabular}{|c|c|c|c|c|c|c|c|c|c|c|c|c|c|c|c|c|c|c|c|c|}
\hline 卢 & $\mid \begin{array}{l}x \\
0 \\
0 \\
0 \\
\tilde{n}\end{array}$ & 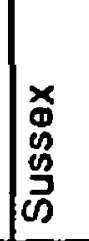 & $\mid \begin{array}{l}x \\
\infty \\
\infty \\
0 \\
\vdots \\
\tilde{\omega}\end{array}$ & 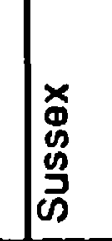 & 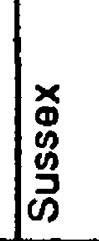 & 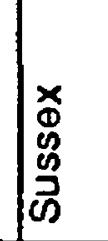 & 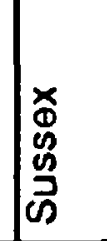 & 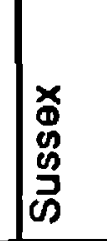 & 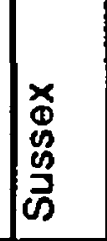 & 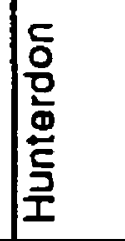 & 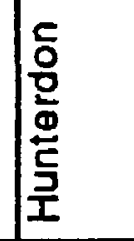 & 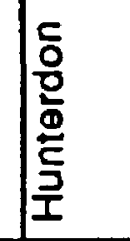 & 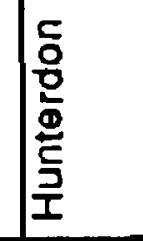 & 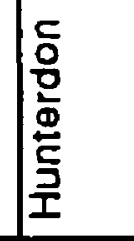 & 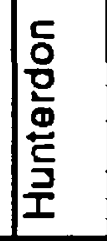 & 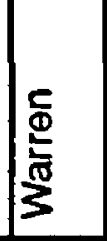 & 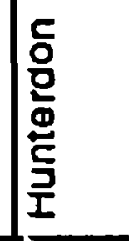 & 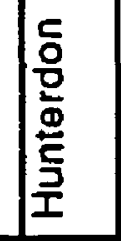 & 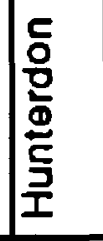 & 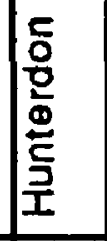 \\
\hline 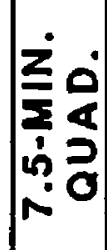 & 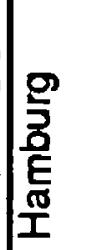 & $\mid \begin{array}{l}0 \\
\frac{0}{5} \\
\text { E⿱ } \\
\frac{0}{1} \\
\frac{0}{1}\end{array}$ & 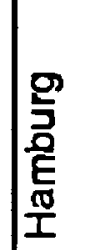 & 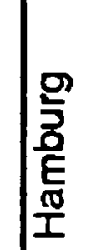 & 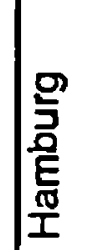 & 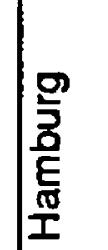 & 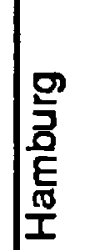 & 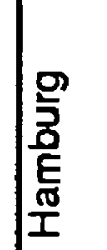 & 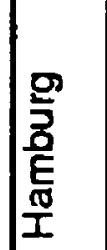 & 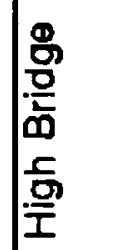 & 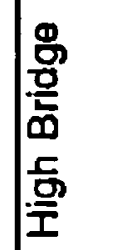 & 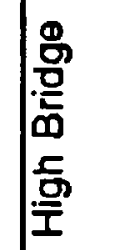 & 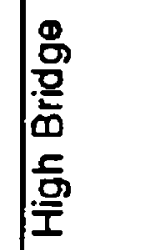 & 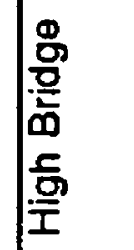 & 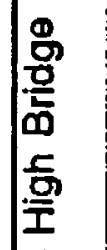 & 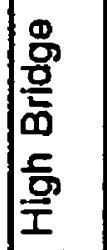 & 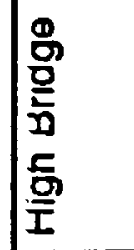 & 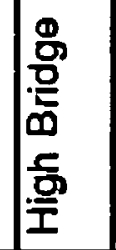 & 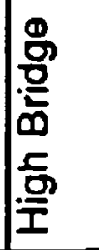 & 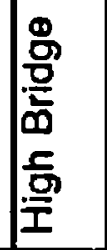 \\
\hline 至 & 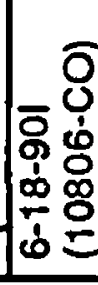 & $\mid \begin{array}{l}0 \\
\delta \\
0 \\
0 \\
0 \\
0 \\
0 \\
0 \\
0 \\
0\end{array}$ & $\stackrel{8}{0}$ & 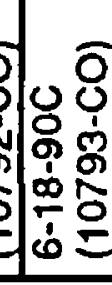 & 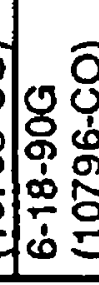 & 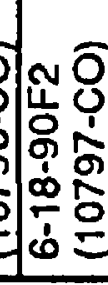 & $16=$ & 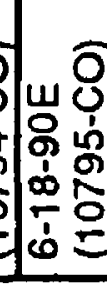 & 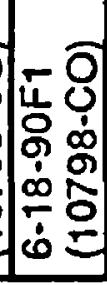 & 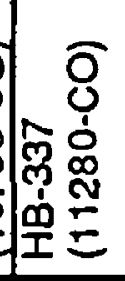 & 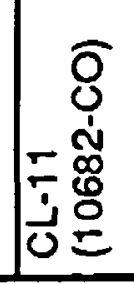 & 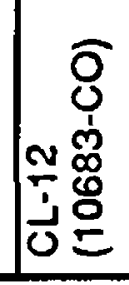 & 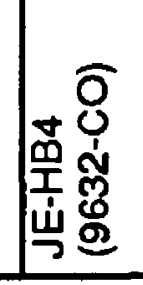 & 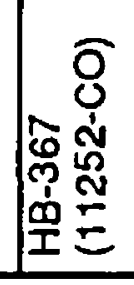 & 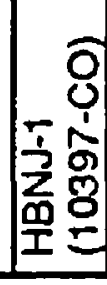 & 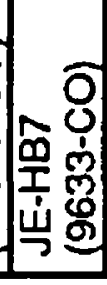 & 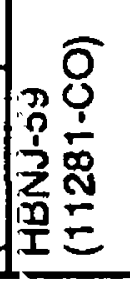 & 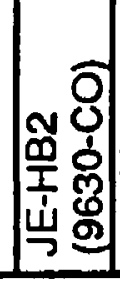 & 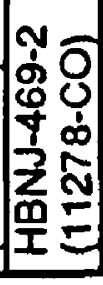 & 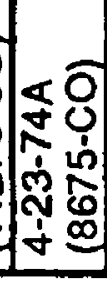 \\
\hline
\end{tabular}




\begin{tabular}{|c|c|c|c|c|c|c|c|c|c|c|c|c|c|c|c|c|c|}
\hline 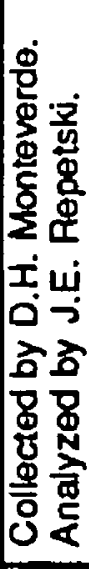 & 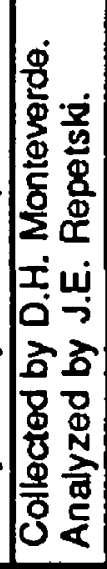 & 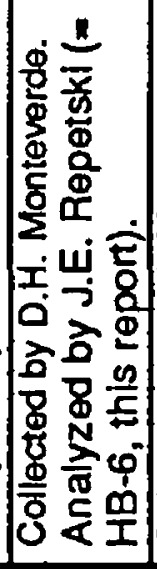 & 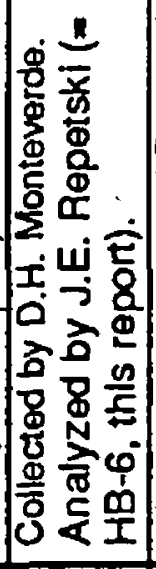 & 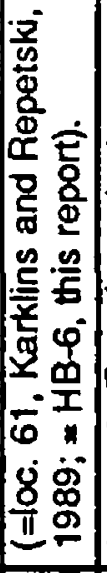 & 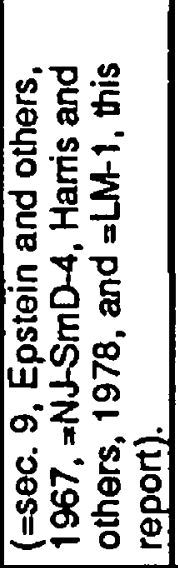 & 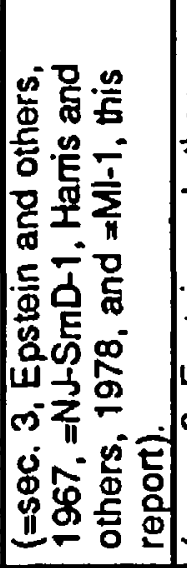 & 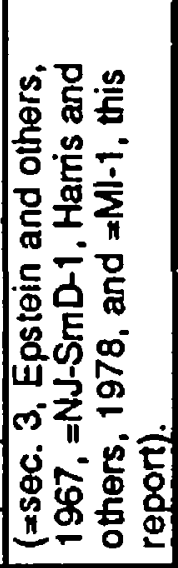 & 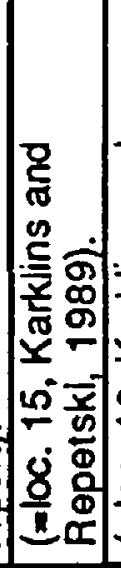 & 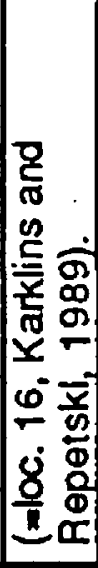 & 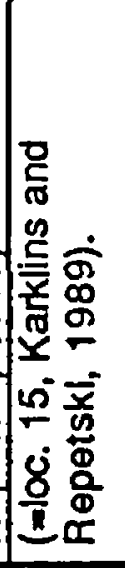 & 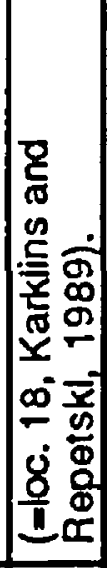 & 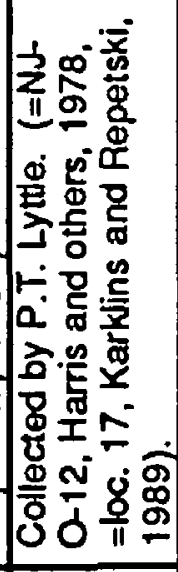 & 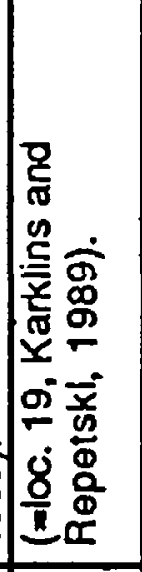 & 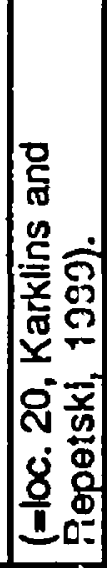 & 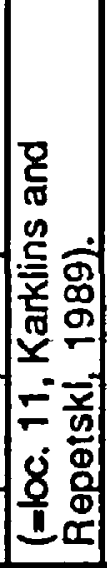 & 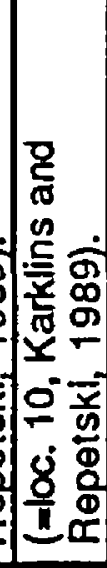 & 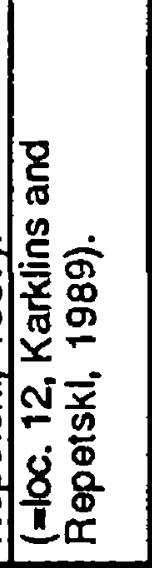 \\
\hline i & $\theta$ & i & \& & & תمן & s & $\infty$ & & $\omega$ & 10 & $\omega$ & $n$ & in & م & in & $n$ & 10 \\
\hline 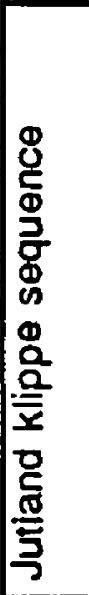 & 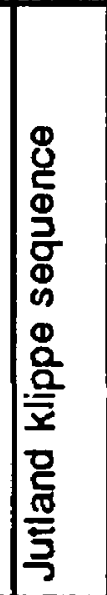 & 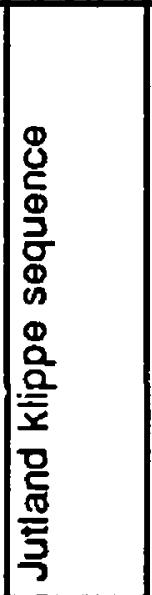 & 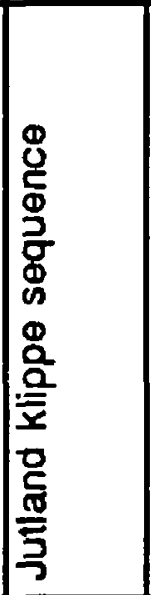 & 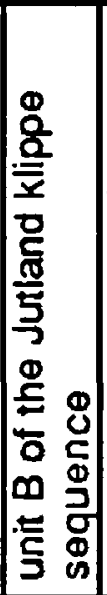 & 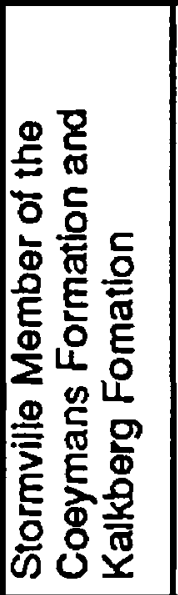 & 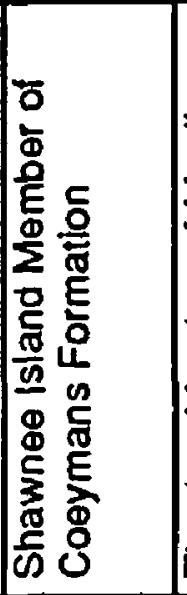 & 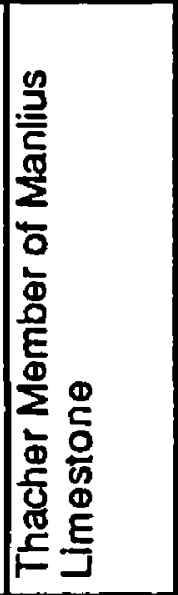 & 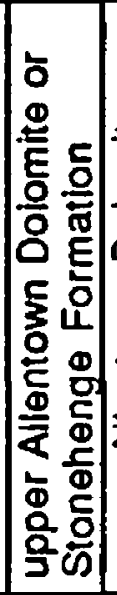 & 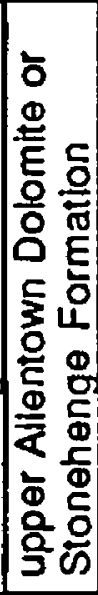 & 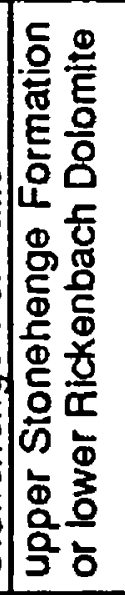 & 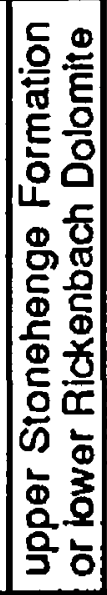 & 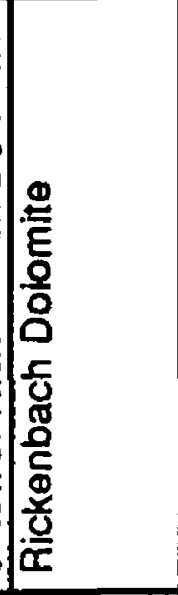 & 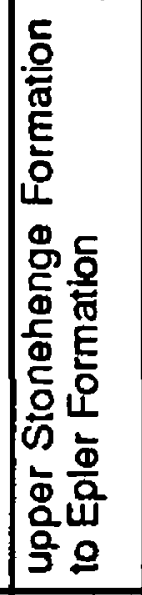 & 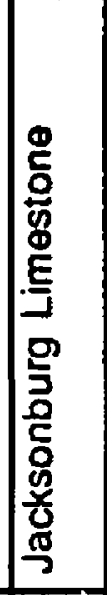 & 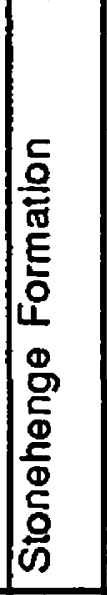 & 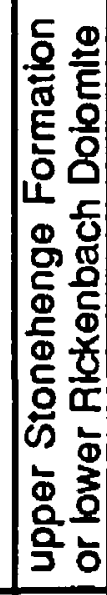 & 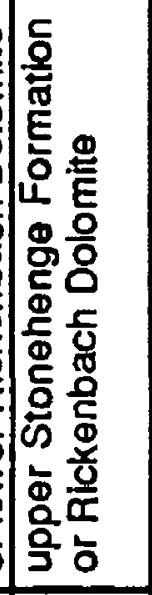 \\
\hline 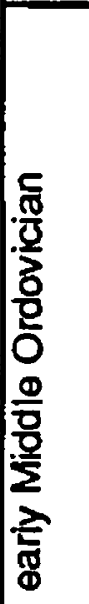 & 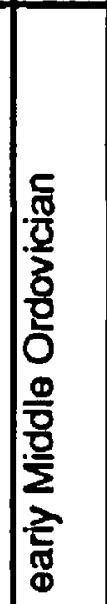 & 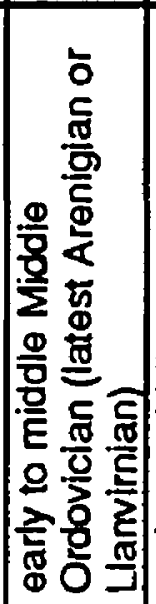 & 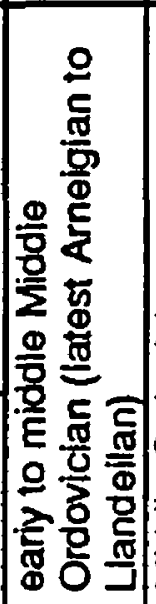 & 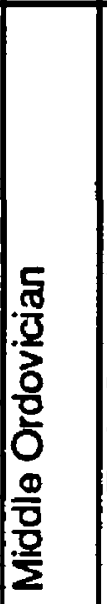 & 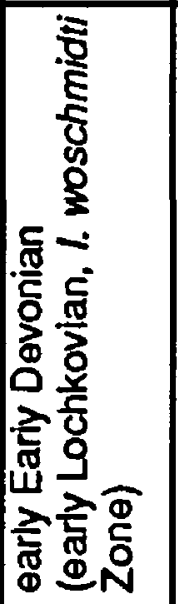 & 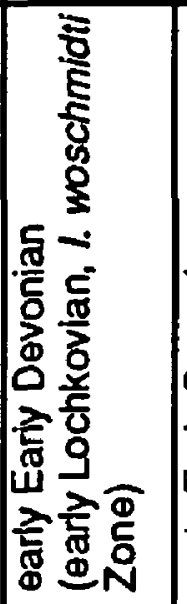 & 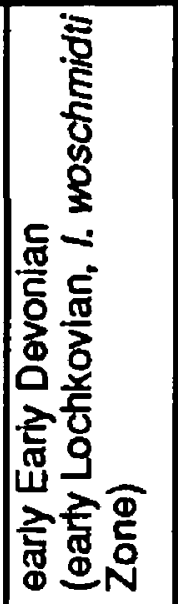 & 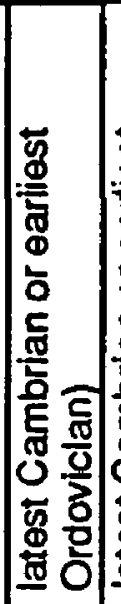 & 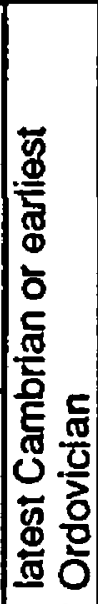 & 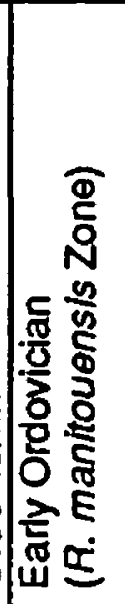 & 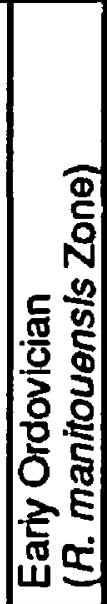 & 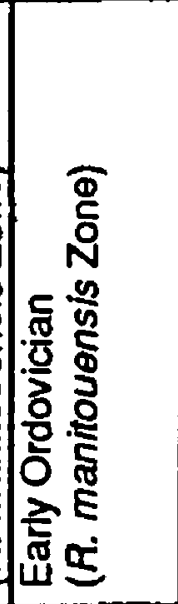 & 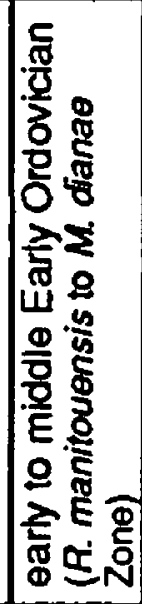 & 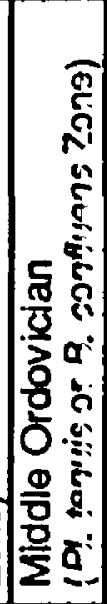 & 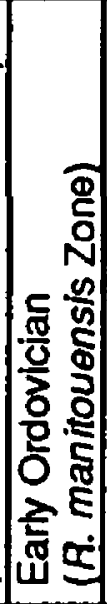 & 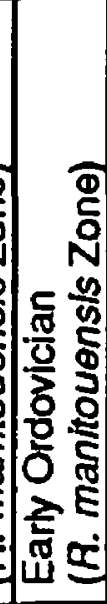 & 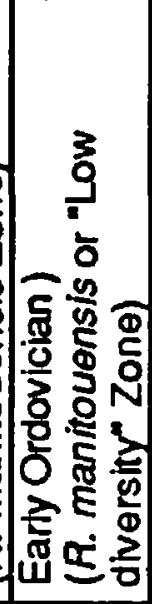 \\
\hline
\end{tabular}

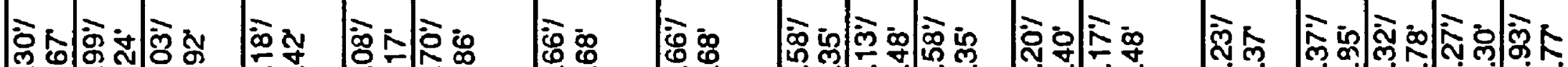

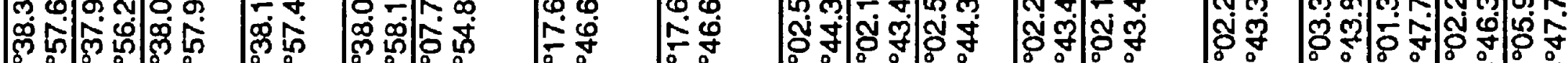

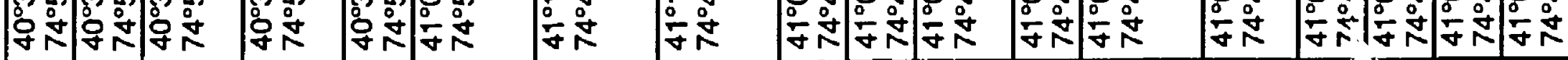

\begin{tabular}{|c|c|c|c|c|c|c|c|c|c|c|c|c|c|c|c|c|c|}
\hline 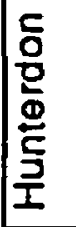 & 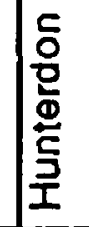 & 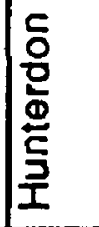 & 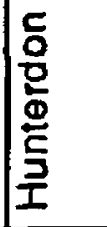 & 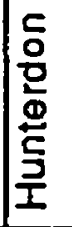 & 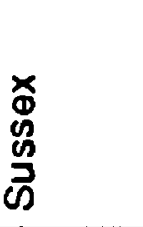 & 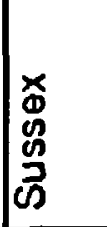 & $\begin{array}{l}x \\
0 \\
\infty \\
\tilde{y} \\
\tilde{\omega} \\
\omega\end{array}$ & 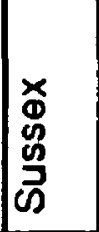 & $\begin{array}{l}x \\
0 \\
0 \\
0 \\
0 \\
\omega \\
\omega\end{array}$ & 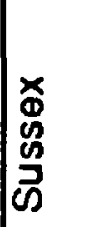 & 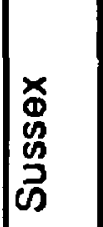 & 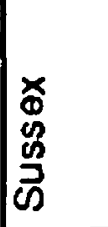 & 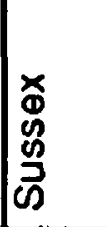 & 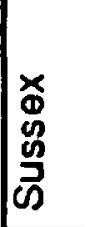 & $\begin{array}{l}\times \\
\Phi \\
\infty \\
心 \\
心\end{array}$ & 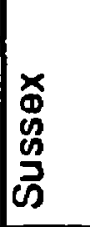 & 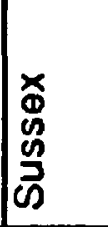 \\
\hline 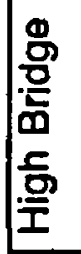 & 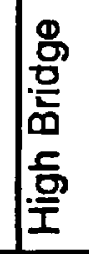 & 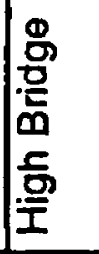 & 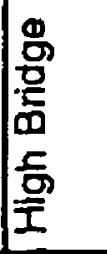 & 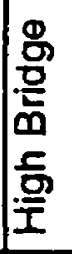 & 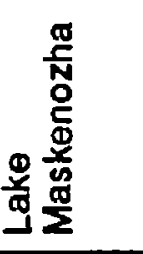 & 总 & $\begin{array}{l}\text { 문 } \\
\text { 意 } \\
\end{array}$ & 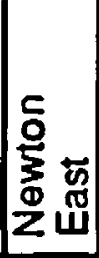 & 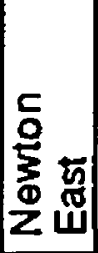 & 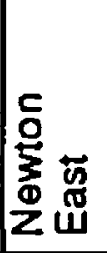 & 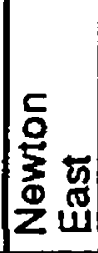 & 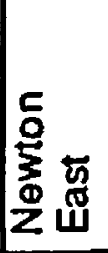 & 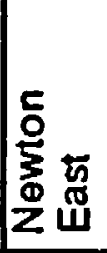 & 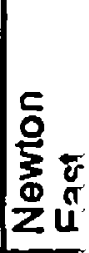 & 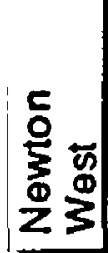 & 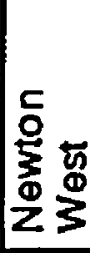 & $\mid \begin{array}{l}5 \\
0 \\
0 \\
0 \\
0 \\
0 \\
0\end{array}$ \\
\hline 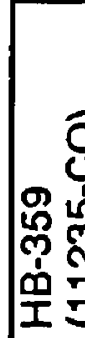 & 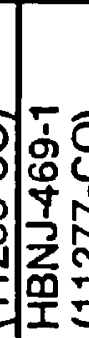 & 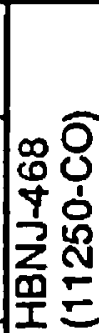 & 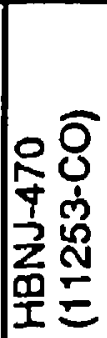 & 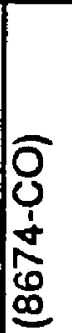 & 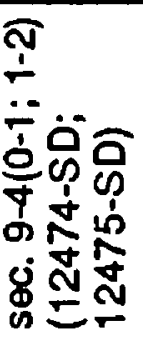 & 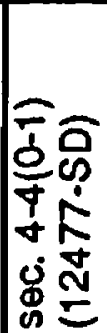 & 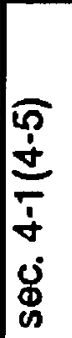 & 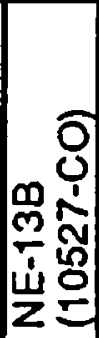 & क़ & 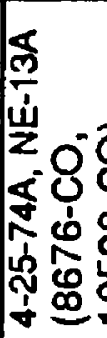 & 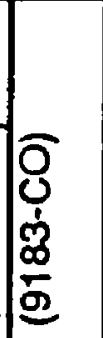 & 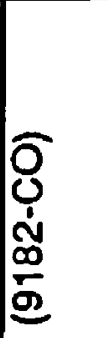 & 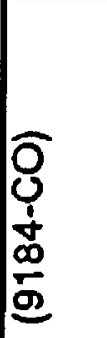 & 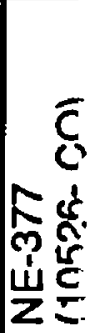 & 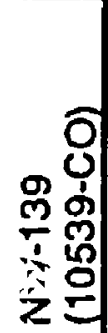 & 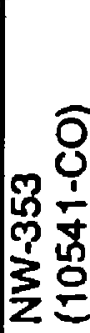 & 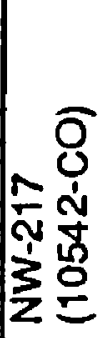 \\
\hline
\end{tabular}




\begin{tabular}{|c|c|c|c|c|c|c|c|c|c|c|c|c|c|c|c|c|c|c|}
\hline 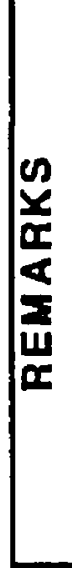 & 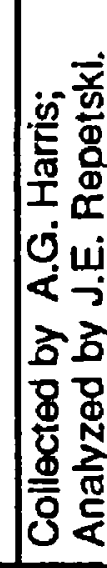 & 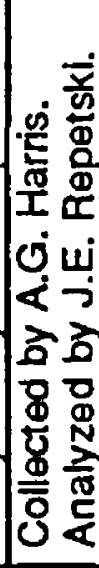 & 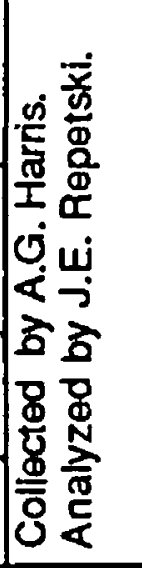 & 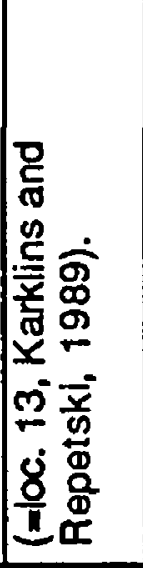 & 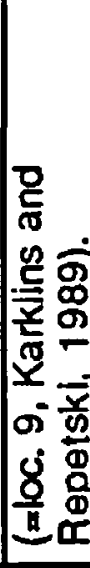 & 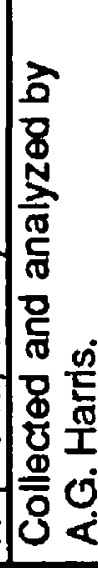 & 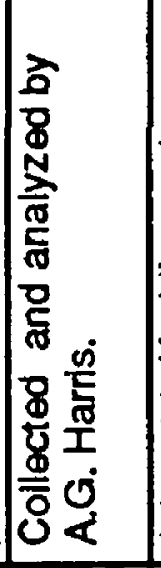 & 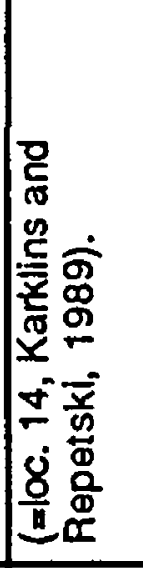 & 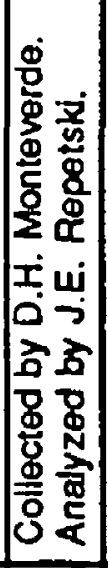 & 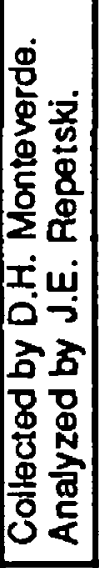 & 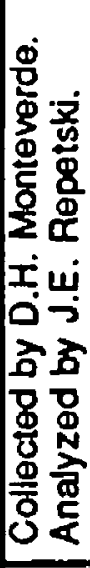 & 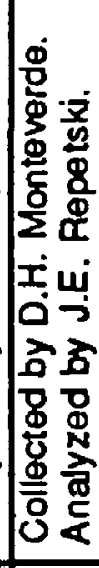 & 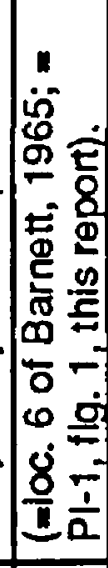 & 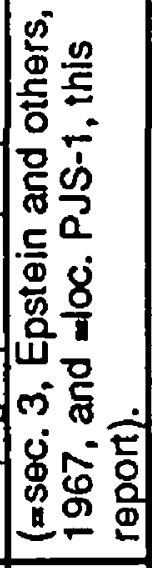 & 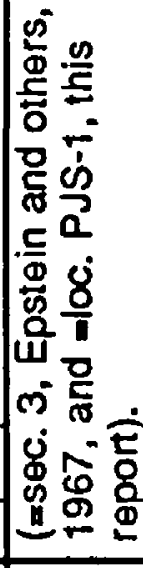 & 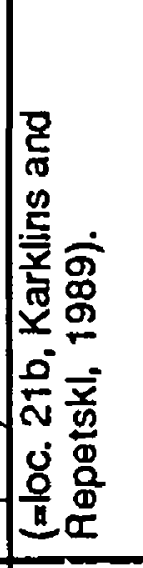 & 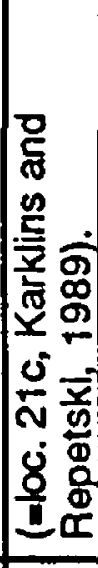 & 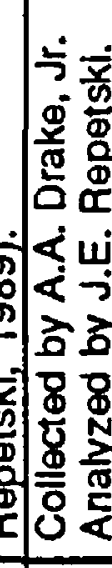 \\
\hline & Ln & م & 10 & n & ما & in & (n) & مـ & $\begin{array}{l}\dot{4} \\
i \\
i\end{array}$ & $\ddot{\star}$ & $\mid \begin{array}{l}n \\
v \\
\dot{x}\end{array}$ & $\mid \begin{array}{l}n \\
v \\
\dot{x} \\
\lambda\end{array}$ & $i_{2}^{+}$ & 100 & مـ & & (n) & |' \\
\hline 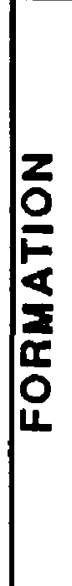 & 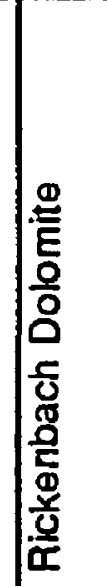 & 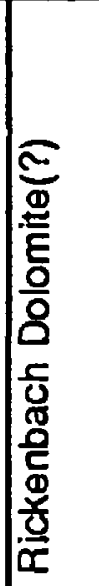 & 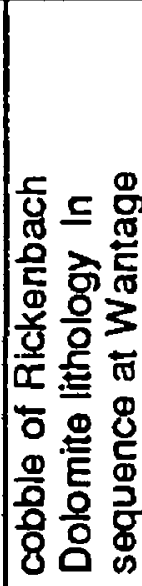 & 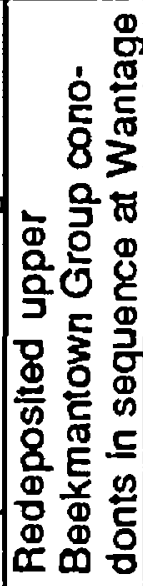 & 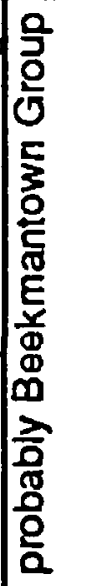 & 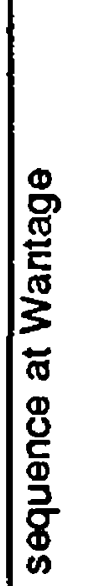 & 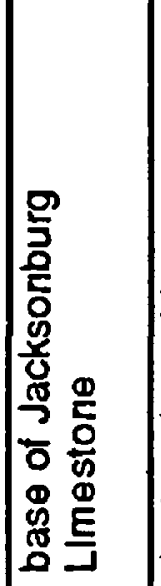 & 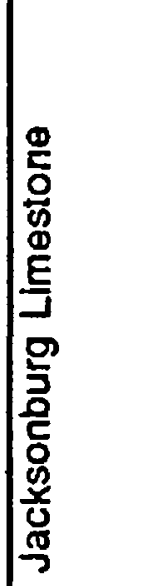 & 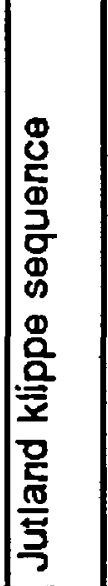 & 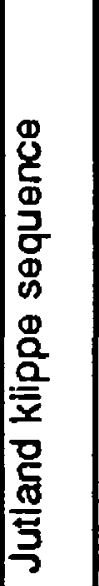 & 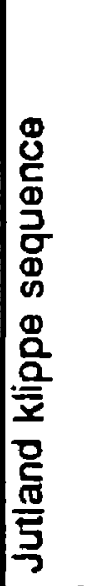 & 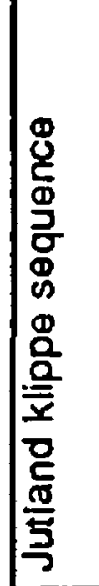 & 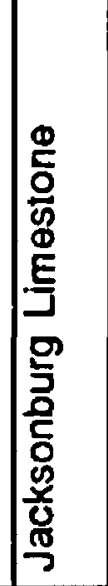 & 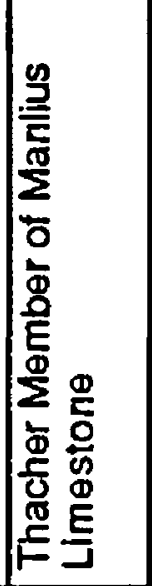 & 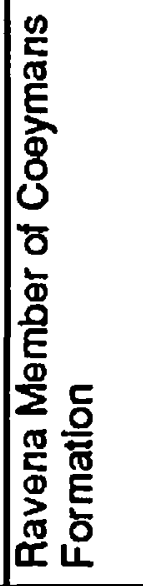 & 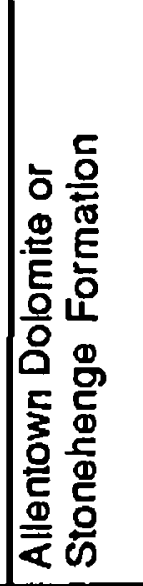 & 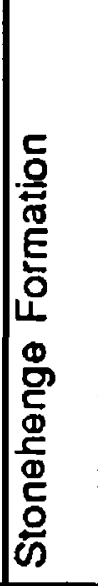 & 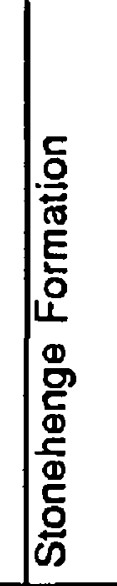 \\
\hline$\underset{\alpha}{u}$ & 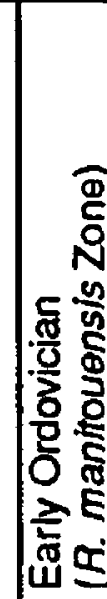 & 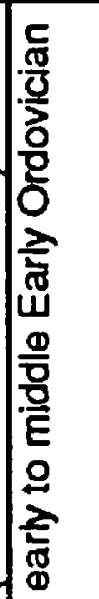 & 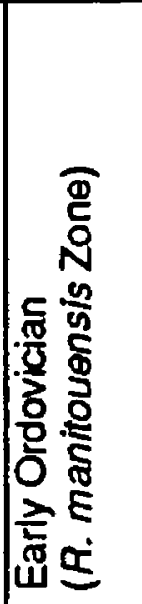 & 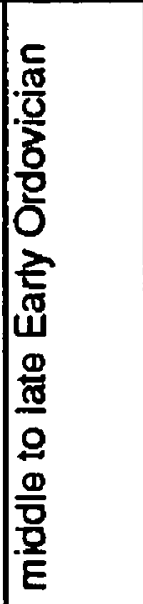 & 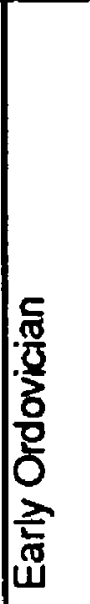 & 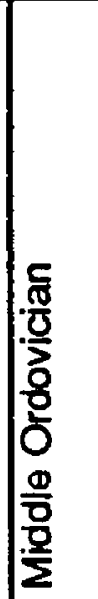 & 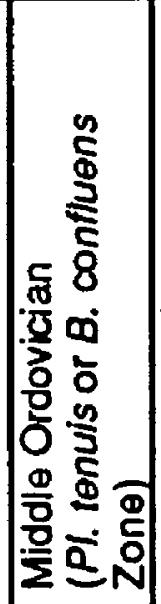 & 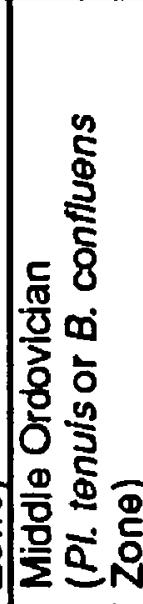 & 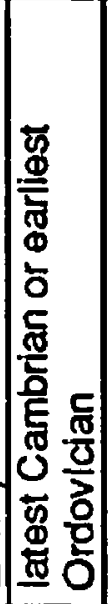 & 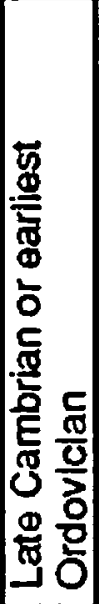 & 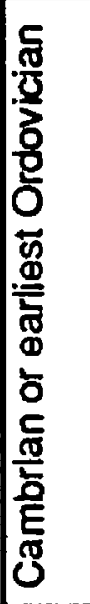 & 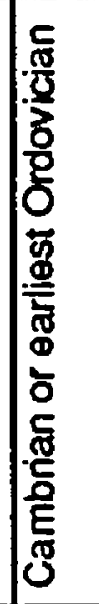 & 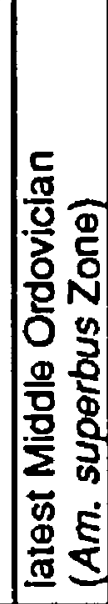 & 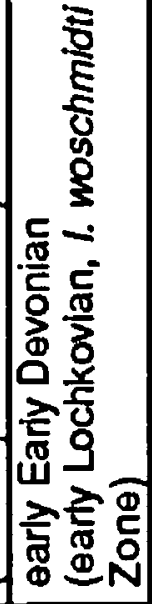 & 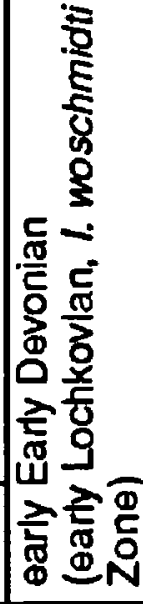 & 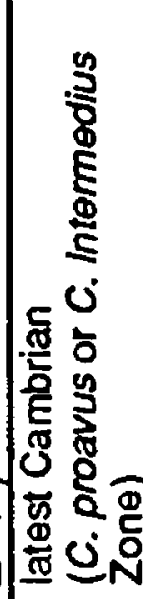 & 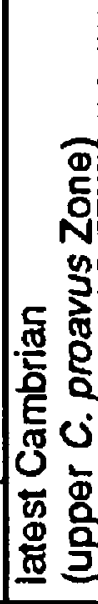 & 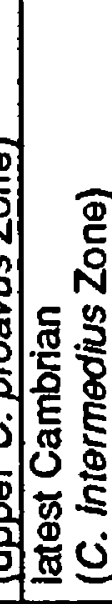 \\
\hline
\end{tabular}

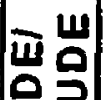

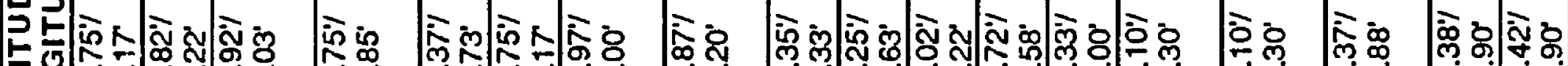

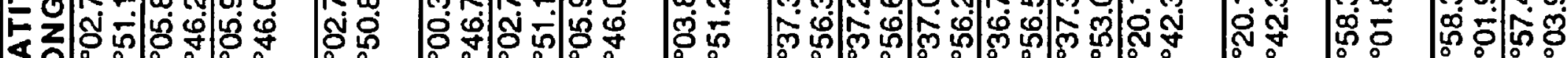

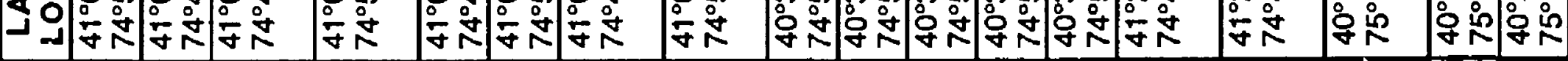

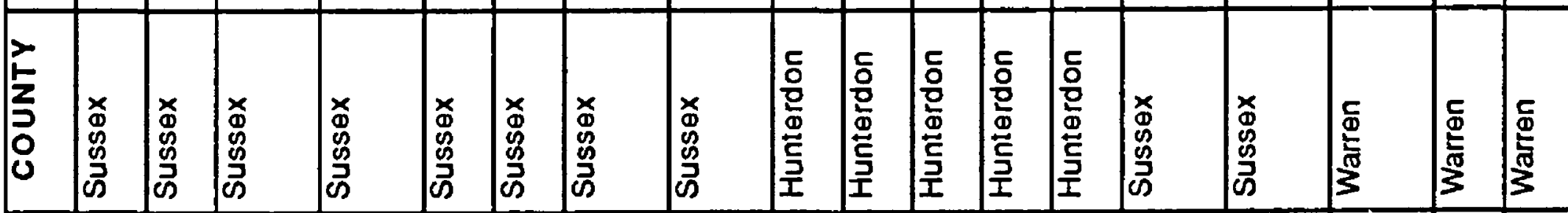

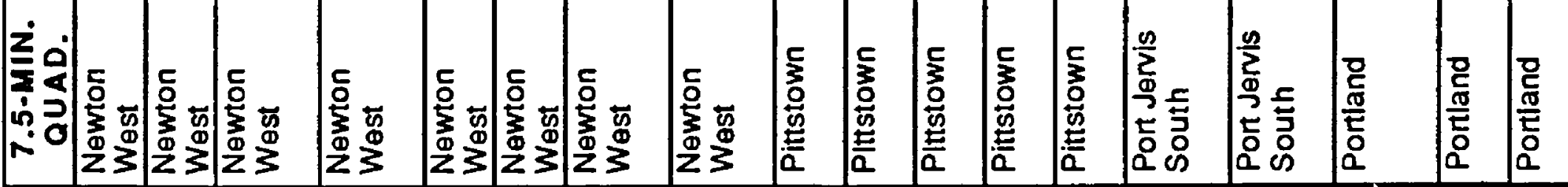

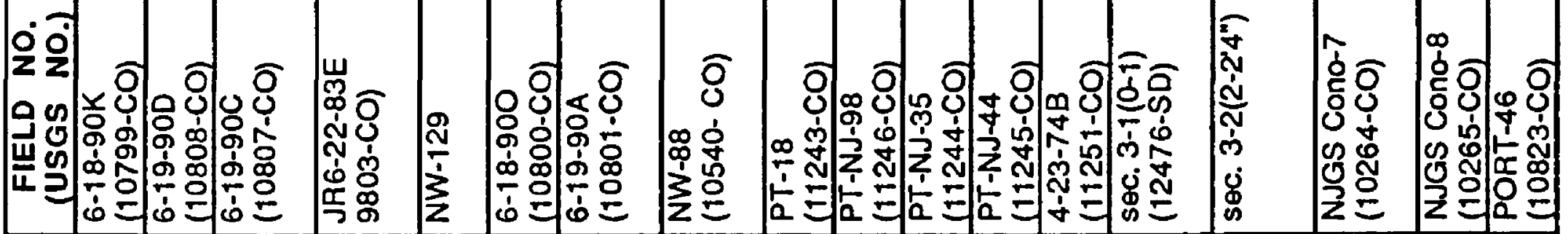




\begin{tabular}{|c|c|c|c|c|c|c|c|c|c|c|c|c|c|c|c|}
\hline 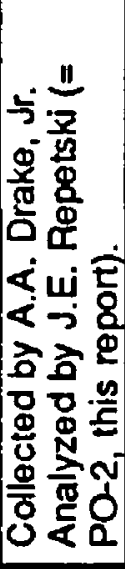 & 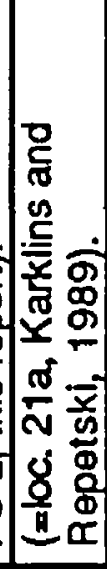 & 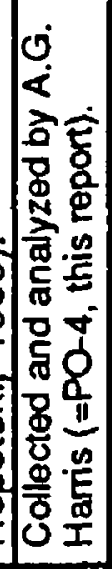 & 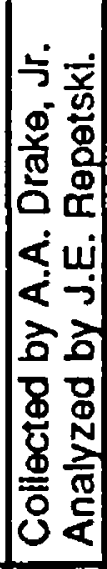 & 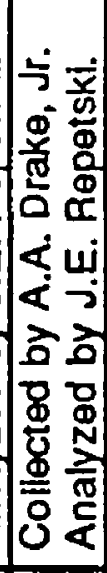 & 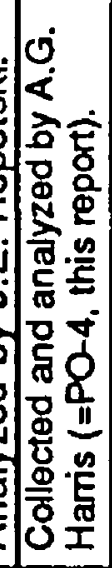 & 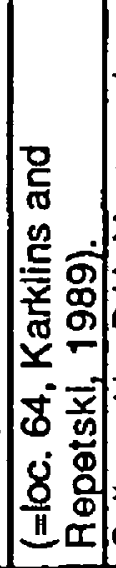 & 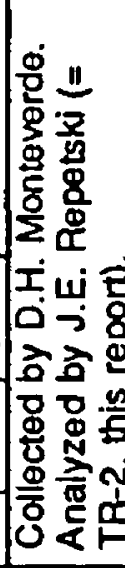 & 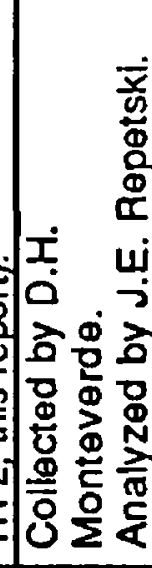 & 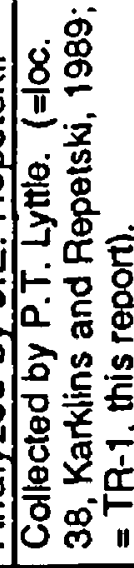 & 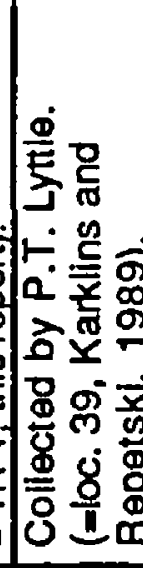 & 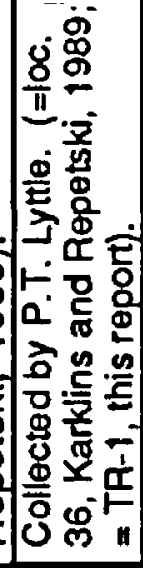 & 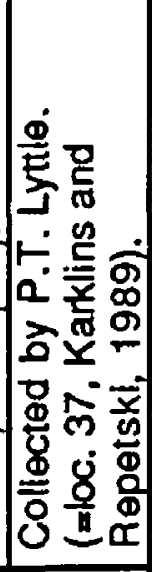 & 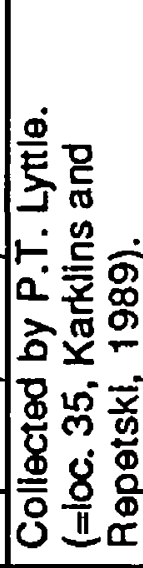 & 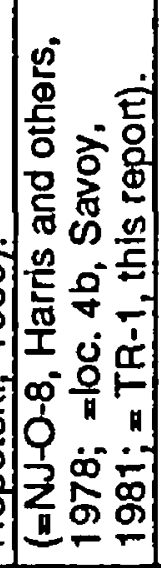 & 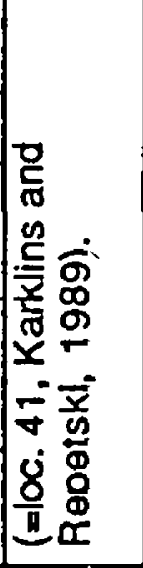 \\
\hline م) & م & ما & م & |م & | & مו & 10 & | & ما & مـ & in & 10 & in & in & in \\
\hline 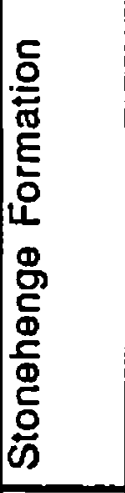 & 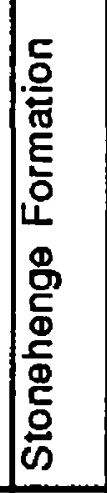 & 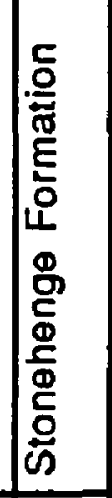 & 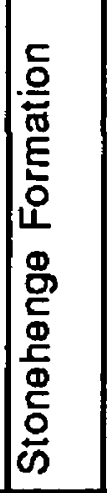 & 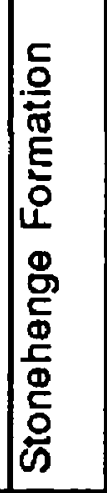 & 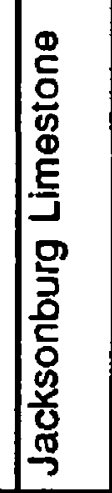 & 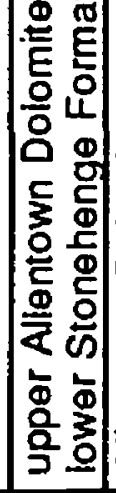 & 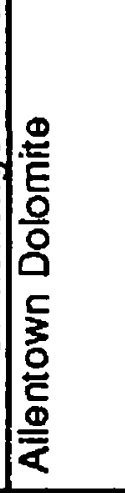 & 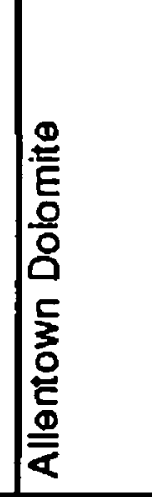 & 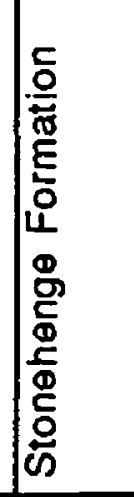 & 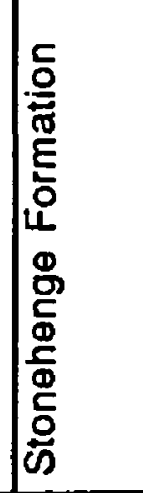 & 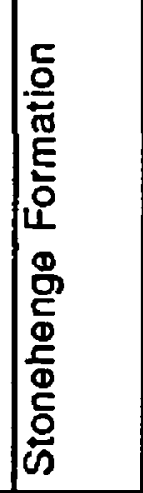 & 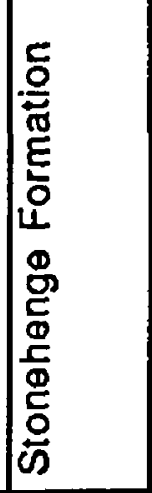 & 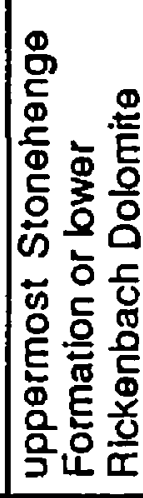 & 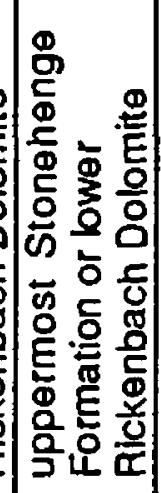 & 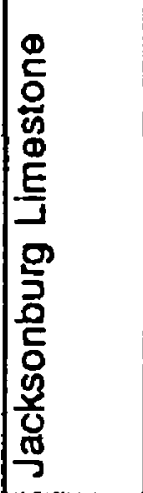 \\
\hline 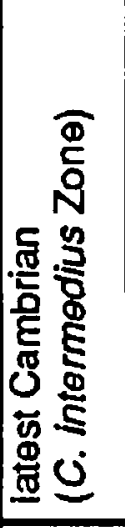 & 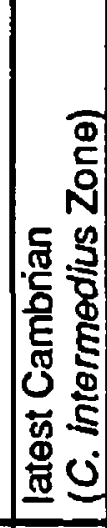 & 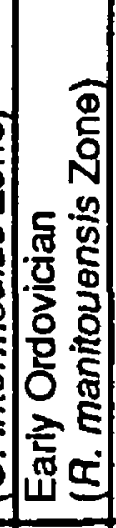 & 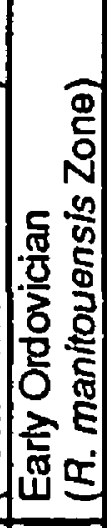 & 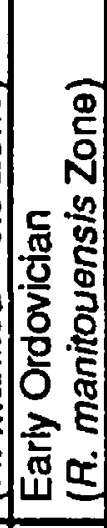 & 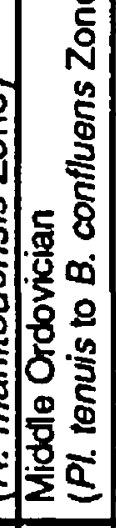 & 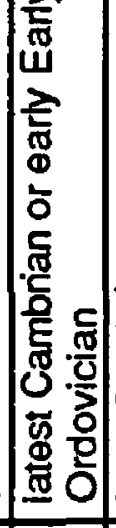 & 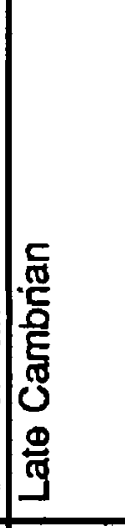 & 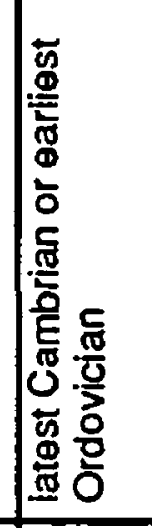 & 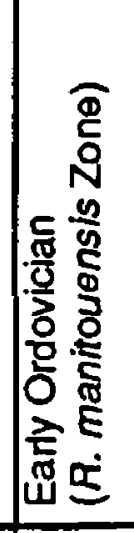 & 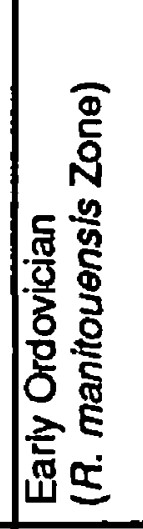 & 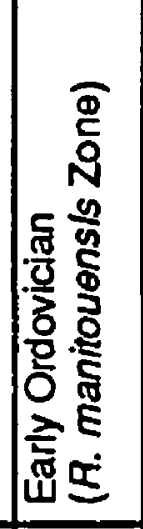 & 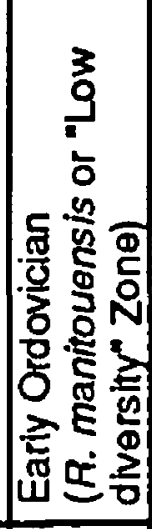 & 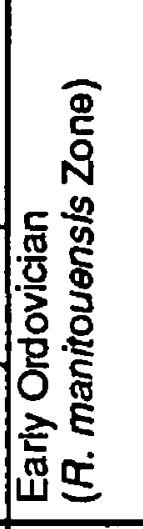 & 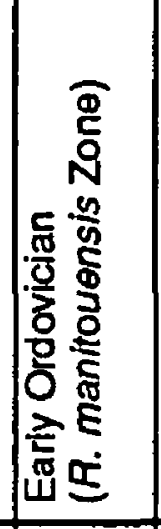 & 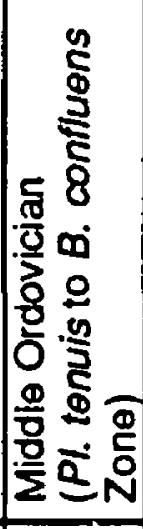 \\
\hline 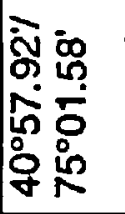 & 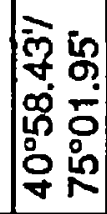 & 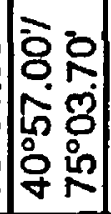 & 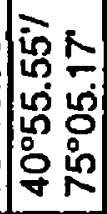 & 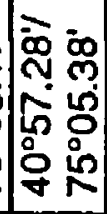 & 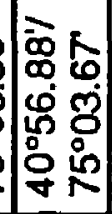 & 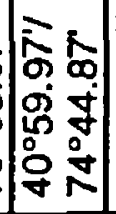 & 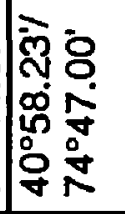 & 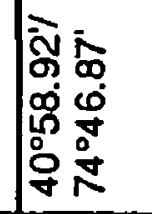 & 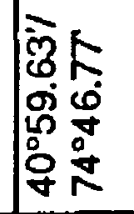 & 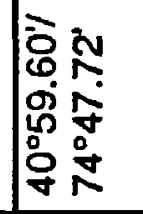 & 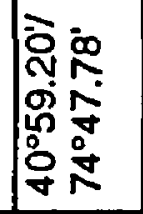 & 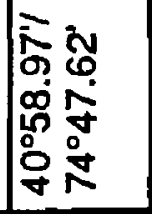 & 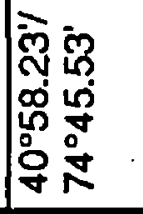 & 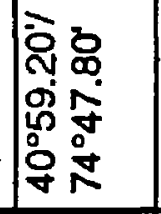 & 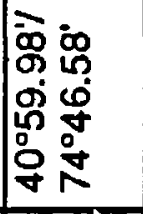 \\
\hline 駡 & 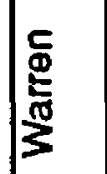 & 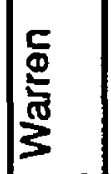 & 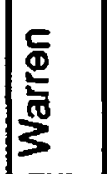 & 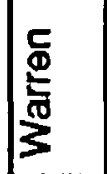 & 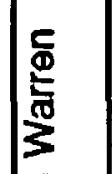 & 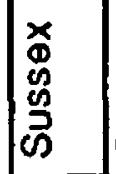 & 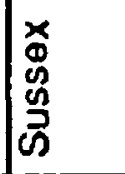 & 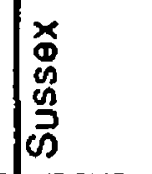 & 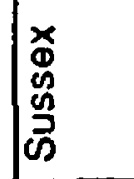 & 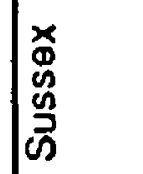 & 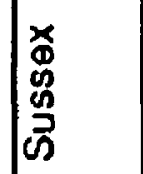 & 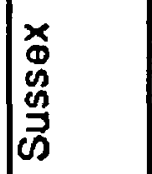 & 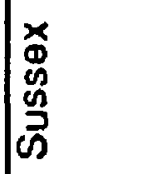 & 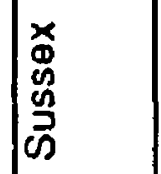 & 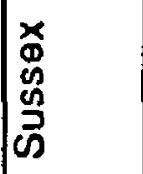 \\
\hline 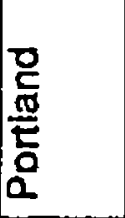 & 总 & $\begin{array}{l}\frac{1}{2} \\
\frac{\pi}{0} \\
\frac{\pi}{0} \\
0 \\
0\end{array}$ & 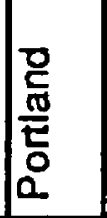 & $\begin{array}{l}\frac{2}{0} \\
\frac{\pi}{\pi} \\
0 \\
0\end{array}$ & 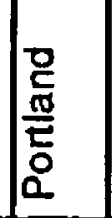 & 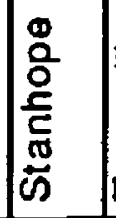 & 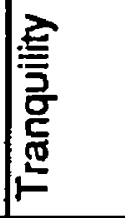 & 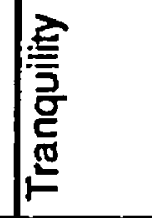 & 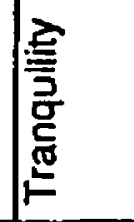 & 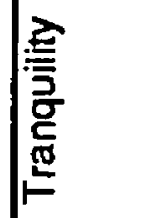 & 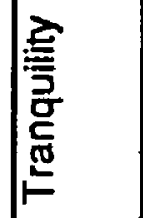 & 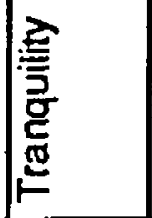 & 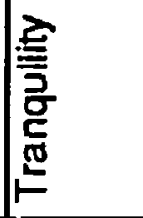 & 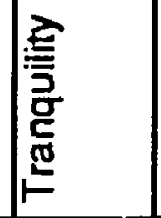 & 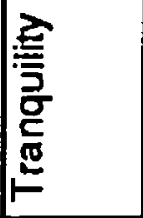 \\
\hline 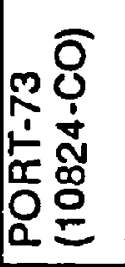 & 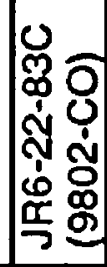 & 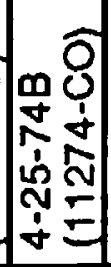 & 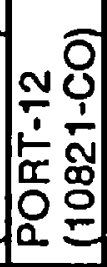 & 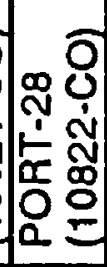 & 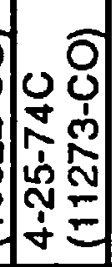 & 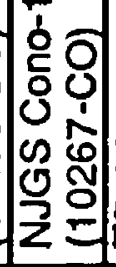 & 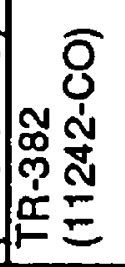 & 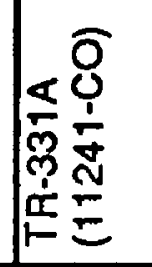 & 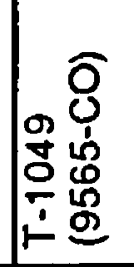 & 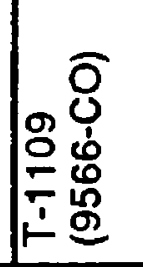 & 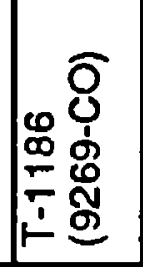 & 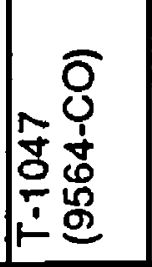 & 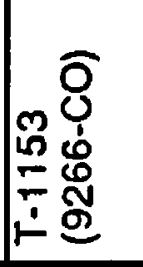 & 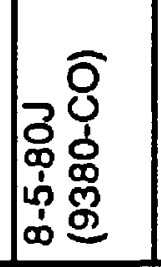 & \begin{tabular}{rl} 
& $\overline{8}$ \\
$\infty$ & 0 \\
0 & 1 \\
0 & 0 \\
\hdashline & 0 \\
& 0 \\
& 0
\end{tabular} \\
\hline
\end{tabular}




\begin{tabular}{|c|c|c|c|c|c|c|c|c|c|c|c|c|c|}
\hline 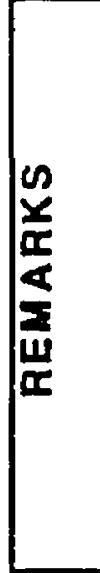 & 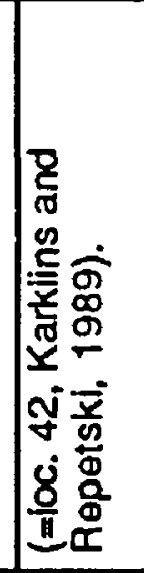 & 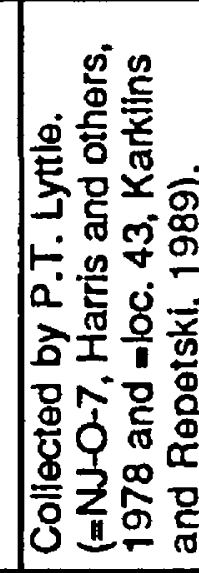 & 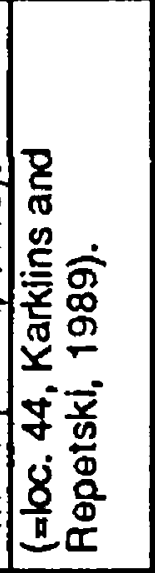 & 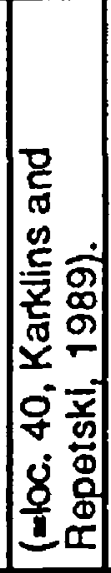 & 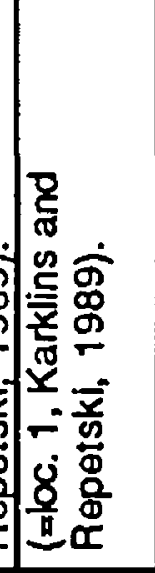 & 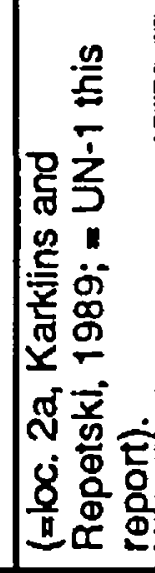 & 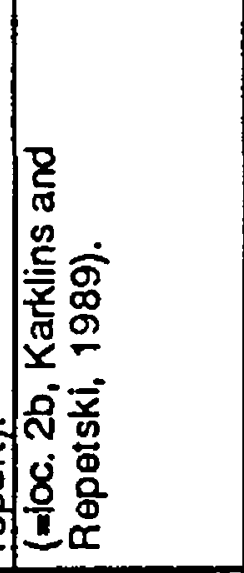 & 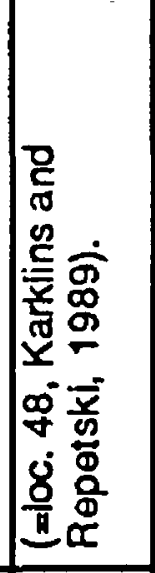 & 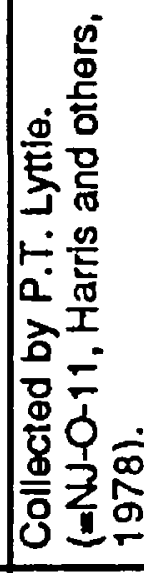 & 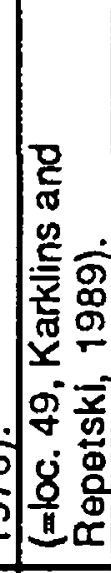 & 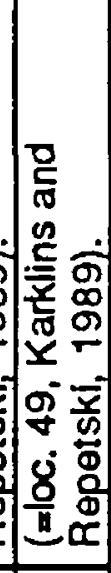 & 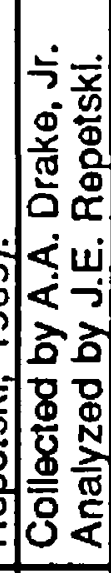 & 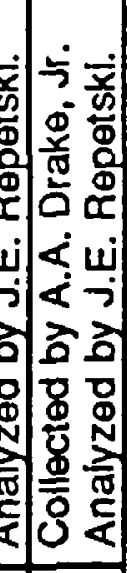 \\
\hline $\bar{\delta}_{0}^{\bar{\alpha}}$ & مـ & مـ & in & 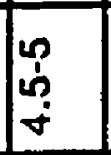 & in & مـ & 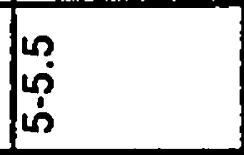 & مـ & ما & L & م & in & in \\
\hline 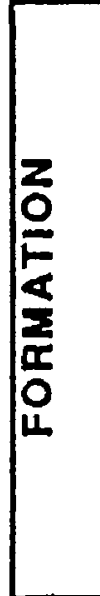 & 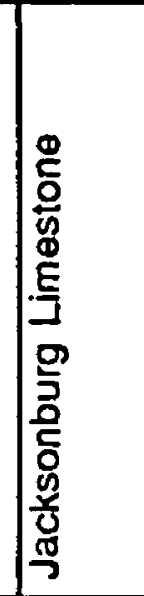 & 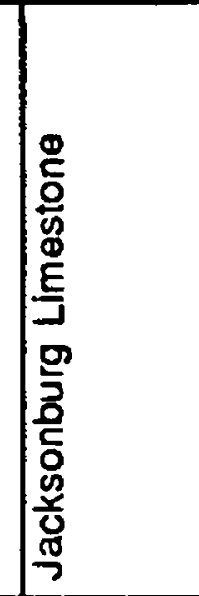 & 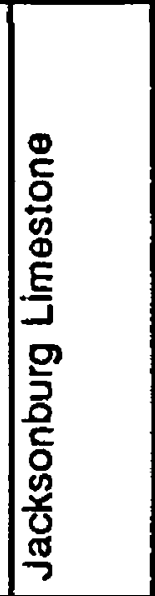 & 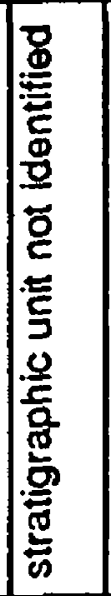 & 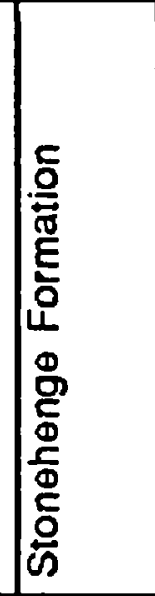 & 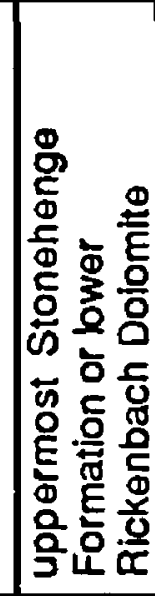 & 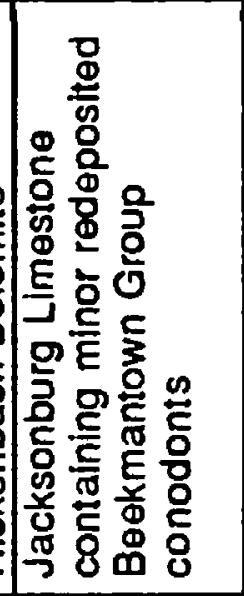 & 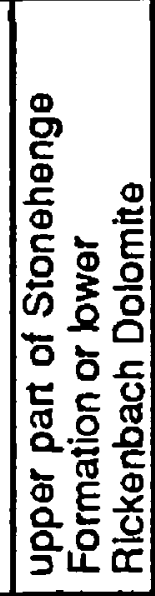 & 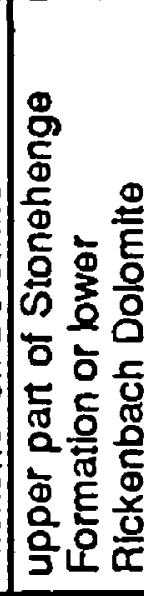 & 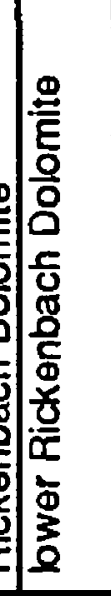 & 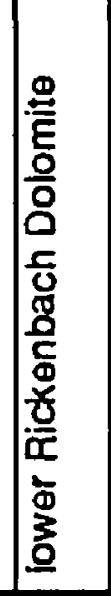 & 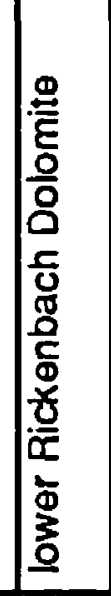 & 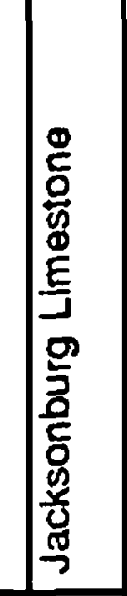 \\
\hline 岁 & 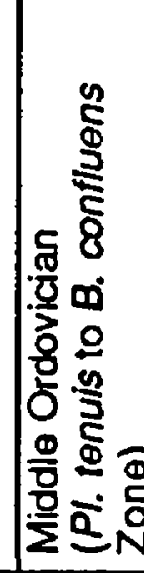 & 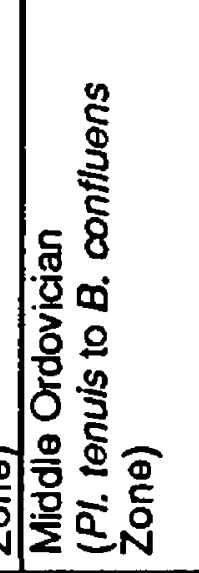 & 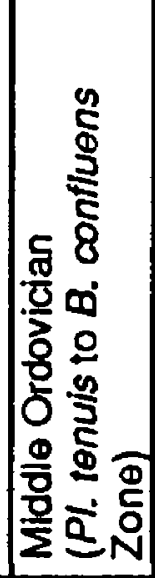 & $\mid$ & 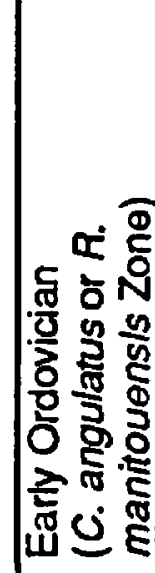 & 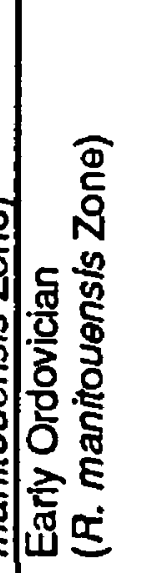 & 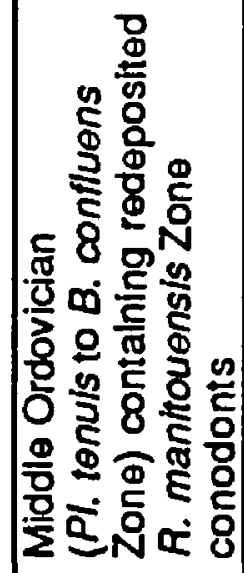 & 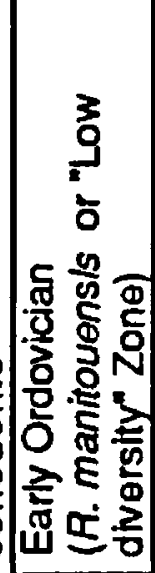 & 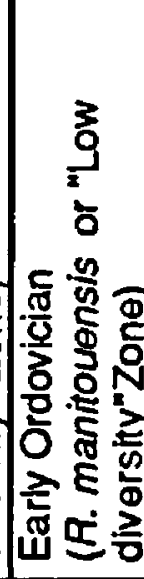 & 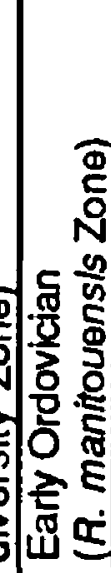 & 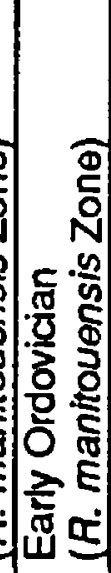 & 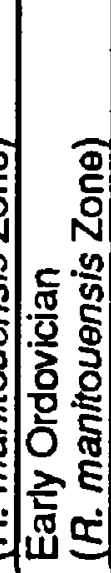 & 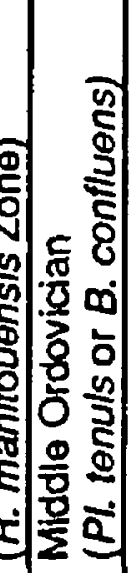 \\
\hline 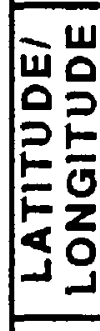 & 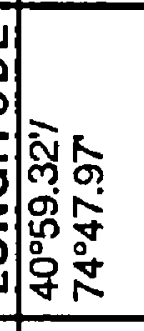 & 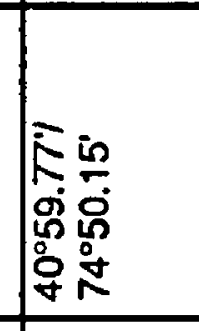 & 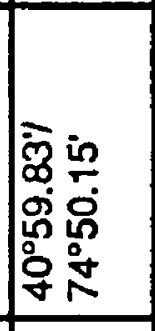 & 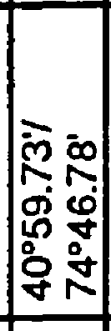 & 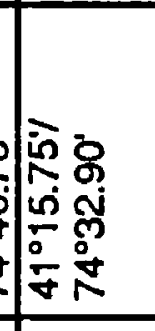 & 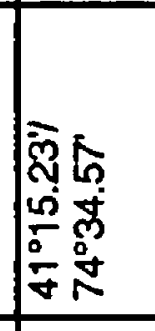 & 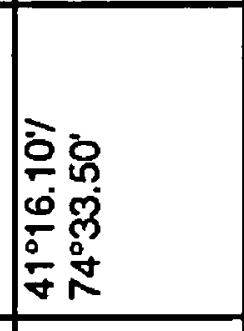 & 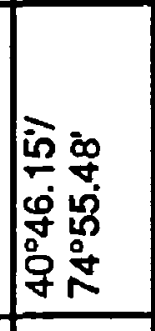 & 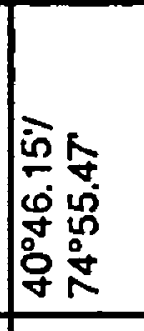 & & 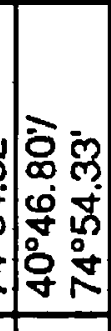 & 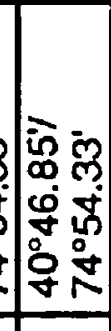 & 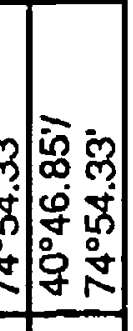 \\
\hline $\mid \begin{array}{l}z \\
z \\
0 \\
0 \\
0 \\
0\end{array}$ & 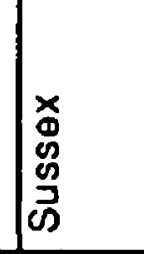 & 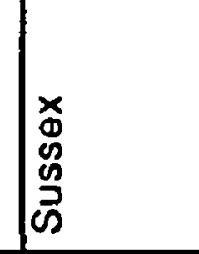 & 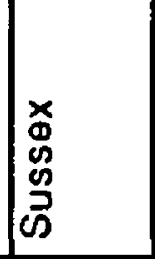 & 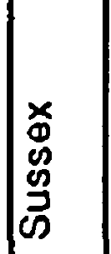 & 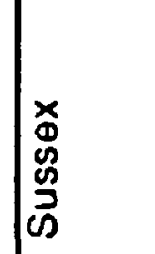 & $\mid \begin{array}{l}x \\
0 \\
y_{0} \\
y_{3} \\
0\end{array}$ & 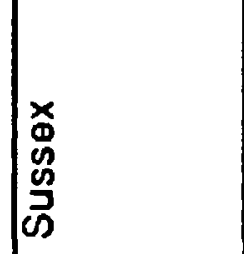 & 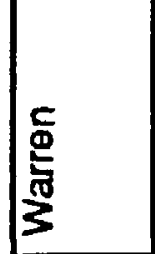 & 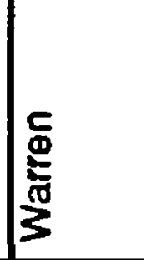 & 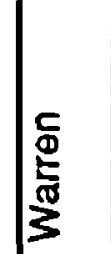 & 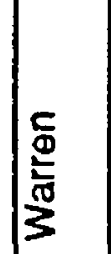 & 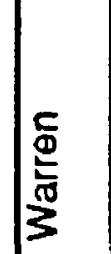 & 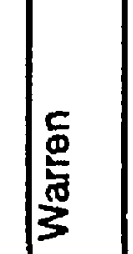 \\
\hline 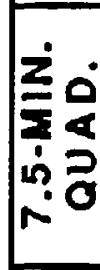 & | & 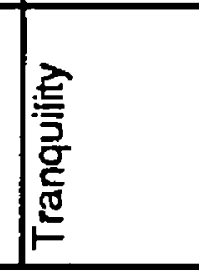 & $\mid$ & 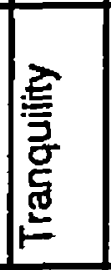 & 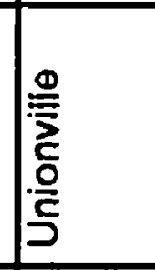 & $\mid \begin{array}{l}\frac{0}{2} \\
\frac{2}{\bar{z}} \\
\frac{0}{5} \\
5\end{array}$ & 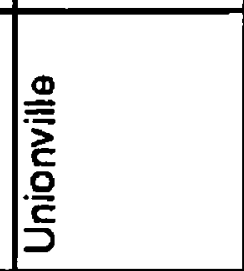 & 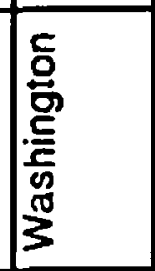 & $\mid$ & 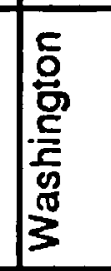 & 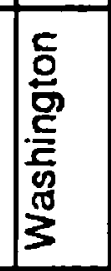 & 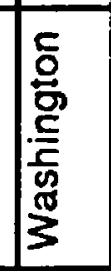 & 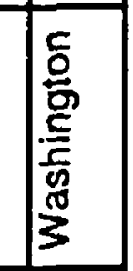 \\
\hline 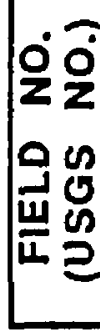 & 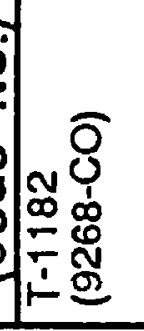 & 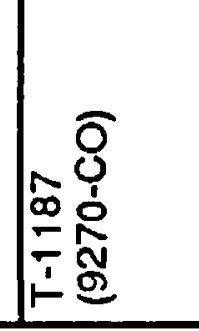 & 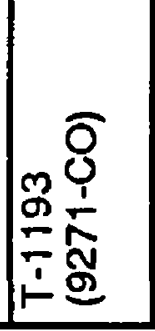 & 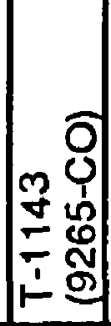 & 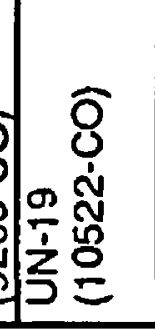 & 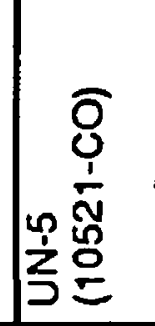 & 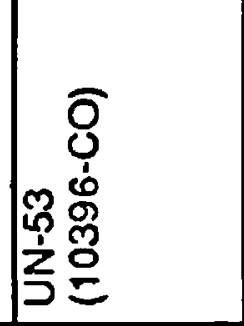 & 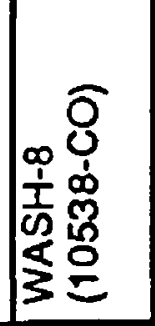 & 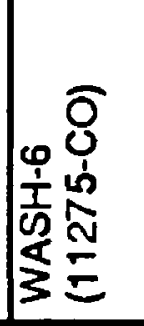 & 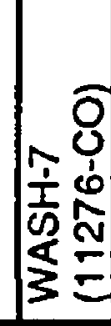 & 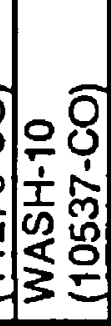 & 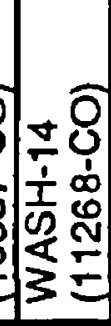 & 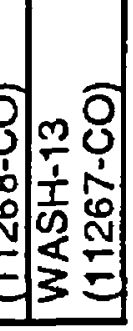 \\
\hline
\end{tabular}




\section{REFERENCES CITED}

Aldridge, R.J., and Theron, J.N., 1993, Conodonts with preserved soft tissue from a new Ordovician Konservat-Lagerstätte: Journal of Micropalaeontology, v. 12, no. 1, p. 113-117.

Barnett, S.G., III, 1965, Conodonts of the Jacksonburg Limestone (Middle Ordovician) of northwestern New Jersey and Pennsylvania: Micropaleontology, v. 11, no. 1, p. 59-80. 1966, Late Cayugan and Helderbergian stratigraphy of southeastern New York and northern New Jersey: Columbus, Ohio, The Ohio State University, Ph.D. dissertation, 217 p.

1971, Biometric determination of the evolution of Spathognathodus remsch?idensis: a method for precise intrabasinal time correlations in the northern Appalachians: Journal of Paleontology, v. 45, no. 2, p. 274-300.

1972, The evolution of Spathognathodus remscheidensis in New York, New Jersey, Nevada, and Czechoslovakia: Journal of Paleontology, v. 46, no. 6, p. 900-917.

Briggs, D.E.G., Clarkson, E.N.K., and Aldridge, R.J., 1983, The conodont animal: Lethaia, v. 16 , p. $1-14$.

Denkler, K.E., 1984, Upper Silurian biostratigraphy of the Andreas quarry, Pennsylvania, in Lash, G.G., Lyttle, P.T., and Epstein, J.B., eds., Geology of an accreted terrare: the eastern Hamburg klippe and surrounding rocks, eastern Pennsylvania: Harrisburg, Pennsylvania, Pennsylvania Bureau of Topographic and Geologic Survey, p. 102-104.

Denkler, K.E., and Harris, A.G., 1988, Conodont-based determination of the Silurian-Devonian boundary in the Valley and Ridge province, northern and central Appalachians: U.S. Geological Survey Bulletin 1837-B, 13 p.

Drake, A.A., Jr., 1969, Precambrian and Paleozoic geology of the Delaware Valley, New JerseyPennsylvania, in Subitsky, Seymour, ed., Geology of selected areas in New Jersey and Pennsylvania: New Brunswick, Rutgers University Press, p. 52-130.

Drake, A.A., Jr., and others, in press, Map of New Jersey--northern bedrock sheet: U.S. Geological Survey Miscellaneous Investigations Series Map, scale 1:100,000.

Epstein, A.G., 1970, Stratigraphy of uppermost Silurian and lowermost Devonian rorks and the conodont fauna of the Coeymans Formation and its correlatives in northeastern Pennsylvania, New Jersey, and southeasternmost New York: Columbus, Ohio, The Ohio State University, Ph.D. thesis, 322 p.

Epstein, A.G., Epstein, J.B., and Harris, L.D., 1977, Conodont color alteration--an index to organic metamorphism: U.S. Geological Survey Professional Paper 995, 27 p.

Epstein, A.G., Epstein, J.B., Spink, W.J., and Jennings, D.S., 1967, Upper Siluriar and Lower Devonian stratigraphy of northeastern Pennsylvania, New Jersey, and southeast 3 rnmost New York: U.S. Geological Survey Bulletin 1243,74 p.

Ethington, R.L., Furnish, W.M., and Markewicz, F.J., 1958, Ordovician conodonts in New Jersey: Journal of Paleontology, v. 32, no. 4, p. 763-769.

Harris, A.G., Dumoulin, J.A., Repetski, J.E., Carter, Claire, 1995, Correlation of Ordovician rocks of northern Alaska, in Cooper, J.D., and others, eds., Ordovician Odysser: Short papers for the Seventh International Symposium on the Ordovician System: Fullerton, Calif., The Pacific Section Society for Sedimentary Geology (SEPM), Book 77, p. 21-26.

Harris, A.G., Harris, L.D., and Epstein, J.B., 1978, Oil and gas data from Paleozoic rocks in the Appalachian basin: maps for assessing hydrocarbon potential and thermal maturity (conodont color alteration isograds and overburden isopachs): U.S. Geological Survey Miscellaneous Investigations Series Map I-917-E, scale 1:2,500,000.

Hobson, J.P., Jr., 1963, Stratigraphy of the Beekmantown Group in southeastern Pennsylvania: Pennsylvania Geological Survey, General Geology Report 37, 331 p.

Karklins, O.L., and Repetski, J.E., 1989, Distribution of selected Ordovician conodont faunas in northern New Jersey: U.S. Geological Survey Miscellaneous Field Studies Map MF-2066, scale $1: 250,000$.

Klapper, G., and Johnson, J.G., 1980, Endemism and dispersal of Devonian conodonts: Journal of Paleontology, v. 54, p. 400-455. 
Mikulic, D.G., Briggs, D.E.G., and Kluessendorf, J., 1985, A soft-bodied fauna: Science, v. 228 , p. 715-717.

Monteverde, D.H., and Herman, G.C., 1989, Lower Paleozoic environments of depาsition and the discontinuous sedimentary deposits atop the Middle Ordovician unconformity in New Jersey, in Grossman, I.G., ed., Paleozoic geology of the Kittatinny Valley and southwest Highlands area, New Jersey: Easton, Pennsylvania, Geological Association of New Jersey 6th Annual Field Conference, p. 95-120.

Oliver, W.A., Jr., and Chlupác, Ivo, 1991, Defining the Devonian: 1979-1989: Lethaia, v. 24, p. 119-122.

Perissoratis, Constantine, Brock, P.W.G., Bruckner, H.K., Drake, A.A., Jr., and Berry, W.B.N., 1979, The Taconides of western New Jersey: new evidence from the Jutland klippe: Geological Society of America Bulletin, Part II, v. 90, p. 154-177.

Rejebian, V.A., Harris, A.G., and Huebner, J.S., 1987, Conodont color and textural alteration: An index to regional metamorphism, contact metamorphism, and hydrothermal alteration: Geological Society of America Bulletin, v. 99, p. 471-479.

Repetski, J.E., Harris, A.G., and Stamm, N.R., 1995, An overview of conodonts f-om New Jersey, in Baker, J.E., ed., Contributions to the paleontology of New Jersey: Trenton, New Jersey, Geological Association of New Jersey.

Savoy, L.E., 1981, Conodont-based age determination of the Lower/Middle Ordovician boundary in the northem Great Valley, southeastern New York-easternmost Pennsylvania: Princeton, New Jersey, Princeton University, Bachelor's thesis, 110 p.

Sweet, W.C., 1984, Graphic correlation of upper Middle and Upper Ordovician roc's, North American Midcontinent Province, U.S.A., in Bruton, D.L., ed., Aspects of the Ordovician System: Palaeontological Contributions from the University of Oslo, No. 29-, p. 23-35. 1988, The Conodonta: morphology, taxonomy, paleoecology, and history of a long-extinct animal phylum: New York, Oxford University Press, Oxford Monographs on Geology and Geophysics no. 10, 212 p. 


\section{PLATES 1-4}

[Specimens on all plates are scanning electron photomicrographs. Specimens are reposited in the U.S. National Museum of Natural History (USNM), Washington, D.C. All figures X 80, unless specified otherwise; all specimens are shown in lateral view unless noter otherwise; localities shown on figure 1] 


\section{PLATE I}

Late Cambrian and Early Ordovician conodonts from northwestern New Jersey

A-D. Variabiloconus bassleri (Furnish). A-C: tectonically deformed elements; from Stonehenge Fm. at loc. TR-1, USGS colln. 9269-CO, USNM 486915-17. D: non-deformed element from dolostone cobble in Jacksonburg Ls. (see text) at loc. BLR-1, USGS 9343-CO, X120, USNI 1486918.

E. Acanthodus uncinatus Furnish. Stonehenge Fm. near loc. PO-1, USGS 10825-CC, USNM 486919.

F, N. "Acanthodus" lineatus (Furnish). Tall-based element (F), from uppermost Stoneh?nge Formation or lower part of the Rickenbach Dolomite, loc. TR-1, USGS 9380-CO, and short-ba:ed element (N), from Stonehenge Formation, loc. PO-1, USGS 10825-CO, USNM 486920, 21.

G. Rossodus? n. sp. Posterolateral view of coniform element; Stonehenge Formation same loc. as fig. N, USNM 486922.

H, I. Scolopodus sulcatus Furnish. H: deformed element from Stonehenge Fm., same loc. as figs. A-C. I: non-deformed element from cobble in base of Jacksonburg Ls., loc. BLR-2, USGS 9350-CO, USNM 486923, 24.

J-L. Rossodus manitouensis Repetski \& Ethington. J: posterolateral view of deformed coniform element from loc. TR-1; K, L: posterolateral and inner lateral views of non-deformed coniform and oistodontiform elements from loc. PO-1. All from Stonehenge Fm., USNM 486925-27.

M. "Oistodus" triangularis Fumish. From cobble in base of Jacksonburg Limestone at loc. BLR-2, USGS 9351-CO, USNM 486928.

O. "Drepanodus" pseudoconcavus Repetski. From Stonehenge Fm. at loc. PO-1, USNM 486929.

P. Chosonodina herfurthi Müller. Posterior view; Stonehenge Formation or Rickenbach Dolomite at loc. UN-1, USGS 10521-CO, USNM 486930.

Q. Clavohamulus? n. sp. A. Postero-basal view; from Stonehenge Formation or Rickenbach Dolomite at loc. EA-1, USGS 10263-CO, USNM 486931.

R. Clavohamulus densus Fumish. Posterior view; Stonehenge Formation at loc. TR-1, USNM 486932.

S. Loxodus bransoni Fumish. From Stonehenge Fm. at loc. HB-3, USGS 10397-CO, USNM 486933.

T. "Paltodus" spurius Ethington \& Clark. From Stonehenge Formation at loc. TR-1, USGS 9565-CO, USNM 486934.

U. Aloxoconus iowensis (Furnish). From cobble in base of Jacksonburg Limestone at loc. BLR-2, USGS 9351-CO, USNM 486935.

V. Phakelodus cf. P. elongatus An. Nearly complete fused cluster of grasping apparatus of protoconodont; from limestone bed in Jutland klippe sequence at loc. PI-2, USGS 11244-CO, X160, USNM 486936.

W. Teridontus nakamurai (Nogami). From cobble in base of Jacksonburg Limestone at loc. BLR-1, USGS 9333-CO, X160, USNM 486937.

$\mathrm{X}, \mathrm{Y}$. Eoconodontus notchpeakensis (Miller). X: rounded element from limestone bed in Jutland klippe sequence at loc. PI-2, USGS 11243-CO, Y: compressed element, from cobble in base of Jacksonburg Limestone at loc. BLR-2, USGS 9359-CO; both X120, USNM 486938, 39.

Z. Proconodontus muelleri Miller. From limestone within Allentown Dolomite at loc. TR-2, USGS 11242-CO, X125, USNM 486940.

AA. Hirsutodontus hirsutus Miller. Limestone in lower part of Stonehenge Formation at loc. PO-2, USGS 10824-CO, X125, USNM 486941.

AB. Cordylodus angulatus Pander. Stonehenge Fm. at loc. HB-3, USGS 10397-CO, USNM 486942.

AC. Hirsutodontus simplex Miller. Same loc. as fig. AA, X125, USNM 486943.

AD. Hirsutodontus rarus Miller. Basal margin of element slightly stretched tectonical\%. Same loc. as fig. AA, X120, USNM 486944.

AE. Cordylodus proavus Müller. Cusp of element broken, displaced posteriorly, and annealed during deformation and diagenesis. From cobble in base of Jacksonburg Limestone at loc. BLR-1, USGS 9333-CO, X160, USNM 486945.

AF. Clavohamulus elongatus Miller. Posterolateral view of advanced morphotype. Same loc. as fig. AA, X120, USNM 486946.

AG. Clavohamulus hintzei Miller. Posterior view of very slightly deformed element. Same loc. as fig. AA, X120, USNM 486947. 
Harris, Repetski, Stamm, and Weary Plate I

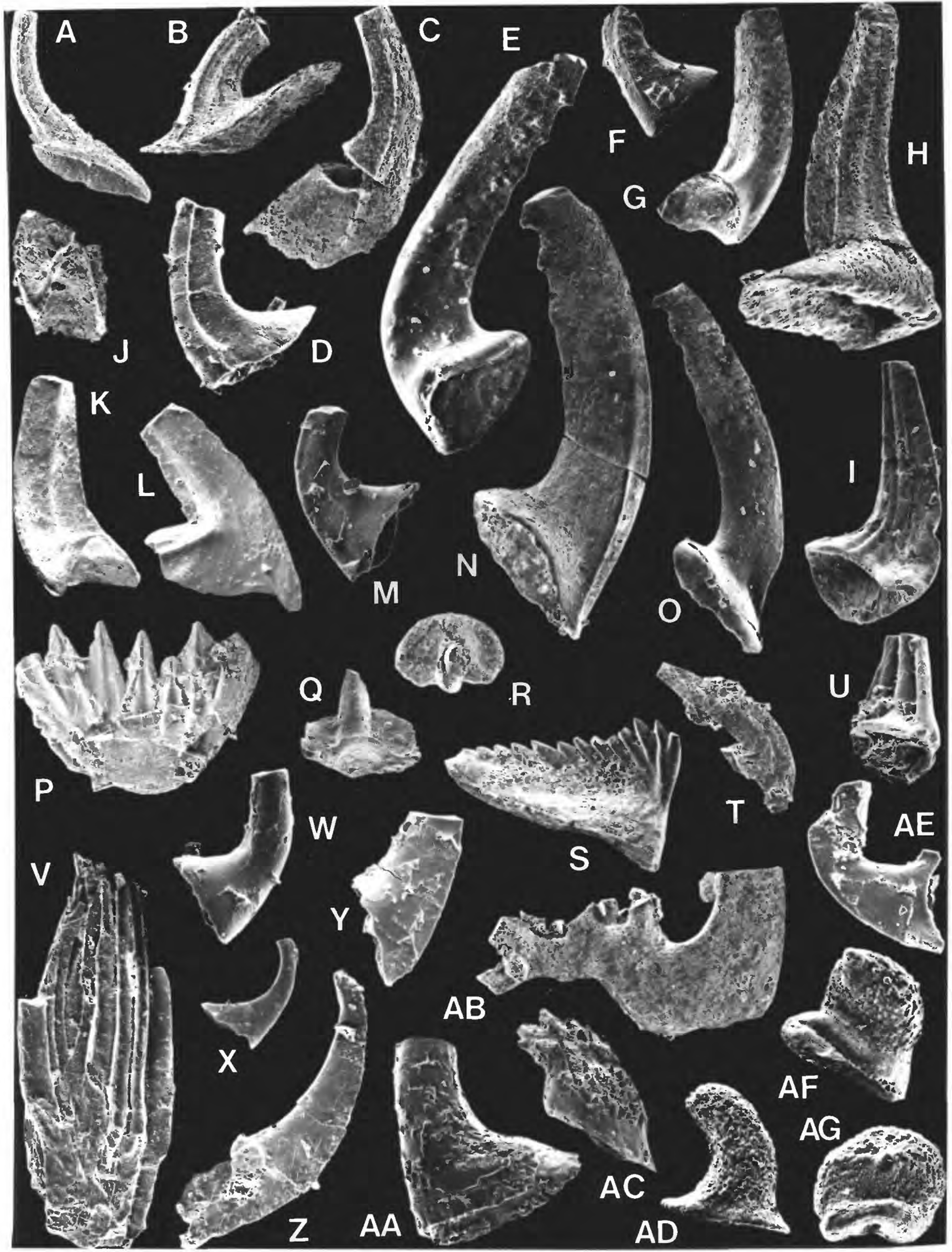




\section{PLATE II}

Early and Middle Ordovician conodonts from northwestern New Jersey

A-E. Periodon flabellum (Lindström). $\mathrm{Pa}, \mathrm{Sb}, \mathrm{Pb}, \mathrm{Sc}$, and $\mathrm{M}$ elements; from a limestone in the Jutland klippe sequence at loc. CH-1, USGS 9105-CO, USNM 486948-52.

F. Periodon aculeatus Hadding (right) and two other ramiform conodont elements on $t$ edding surface of siltstone from Jutland klippe, loc. HB-6, USGS 11250-CO, X50, USNI 1 486953.

G. Cordylodus intermedius Furnish. This species is an index for the lower Ibexian. From a limestone in the Jutland klippe sequence at loc. HB-4, USGS 11252-CO, USNM 486954.

H. Cordylodus lindstromi Druce \& Jones. This is a key index species in the lower Ibevian. Same loc. as fig. G, USNM 486955.

I,J. Protoprioniodus sp. Oistodontiform (M) and "elongatiform" elements. Same loc. as figs. AE, USNM 486956, 57.

K,L. Paroistodus parallelus (Pander). Oistodontiform and drepanodontiform element S Same loc. as figs. A-E, USNM 486958, 59.

M. Protopanderodus cf, $P$. rectus (Lindström). Same loc. as figs. A-E, USNM 48696n.

N. Oneotodus costatus Ethington \& Brand. Posterolateral view of poorly preserved element. From Epler Formation at loc. BLR-3, USGS 9442-CO, USNM 486961.

O. Diaphorodus delicatus (Branson \& Mehl). Sc element. Species is an auxiliary guid s for the Oepikodus communis Zone in warm, restricted, shallow-water facies. Epler Formation, same loc. as fig. N, USNM 486962.

P. Tropodus comptus (Branson \& Mehl). Specimen shows traces of annealed dolomit: rhombs. Epler Formation, same loc. as fig. N, USNM 486963.

Q. Oepikodus communis (Ethington \& Clark). Ramiform (S) element. From cobble in base of Jacksonburg Limestone at loc. BLR-1, X160, USNM 486964.

R. Protoprioniodus aranda Cooper. Anterolateral view. Epler Formation, same loc. a? fig. N, USNM 486965.

S. "Scolopodus" emarginatus Barnes \& Tuke. Posterolateral view. Epler Formation, same loc. as fig. N, USNM 486966.

T. Chionoconus avangna Smith. Epler Formation, same loc. as fig. N, USNM 486967.

U-W. Scolopodus floweri Repetski. Scandodontiform (V) and two multicostate element? Species is an index in the middle Ibexian. Limestone in the Epler Formation, loc. BE-1, USGS 9637-CO, USNM 486968-70.

$\mathrm{X}, \mathrm{Y}$. Macerodus dianae Fåhraeus \& Nowlan. A key species in the middle Ibexian. Element $\mathrm{Y}$ stretched and broken. Epler Formation, same loc. as figs. U-W, X120, USNM 486971,72.

Z,AA. Clavohamulus? new species B. Anterior and posterior views. Epler Formation, same loc. as figs. U-W, X160, USNM 486973,74.

AB,AC. Protopanderodus leei Repetski. Posterolateral views of symmetrical (AB) and asymmetrical (AC) elements. Fig. AB from Epler Formation at loc. BE-1, USGS 9 $337-\mathrm{CO}$; fig. AC from loc. BA-1, Epler Formation on Pennsylvania side of Delaware River, both X120, USNM 486975, 76.

AD,AE. Striatodontus? prolificus Ji \& Barnes. Posterolateral (AD) and posterior (AE) v:ews. Epler Formation, same loc. as figs. U-W, USNM 486977, 78.

AF. Parapanderodus striatus (Graves \& Ellison). Upper view, showing cross-section of cusp. Epler Formation, same loc. as fig. AC, X240, USNM 486979.

AG. Colaptoconus quadraplicatus (Branson \& Mehl). This is one of the most common sjecies in the upper Ibexian rocks of the North American Midcontinent Faunal Realm. Epler Formation, same loc. as fig. N, USNM 486980.

AH. Rossodus? n. sp. Posterior view of coniform element. From redeposited cobble of Epler Formation or Rickenbach Dolomite lithology, in Jacksonburg Limestone at loc. BLP-1, USGS 9340-CO, X160, USNM 486981. 
Harris, Repetski, Stamm, and Weary Plate II

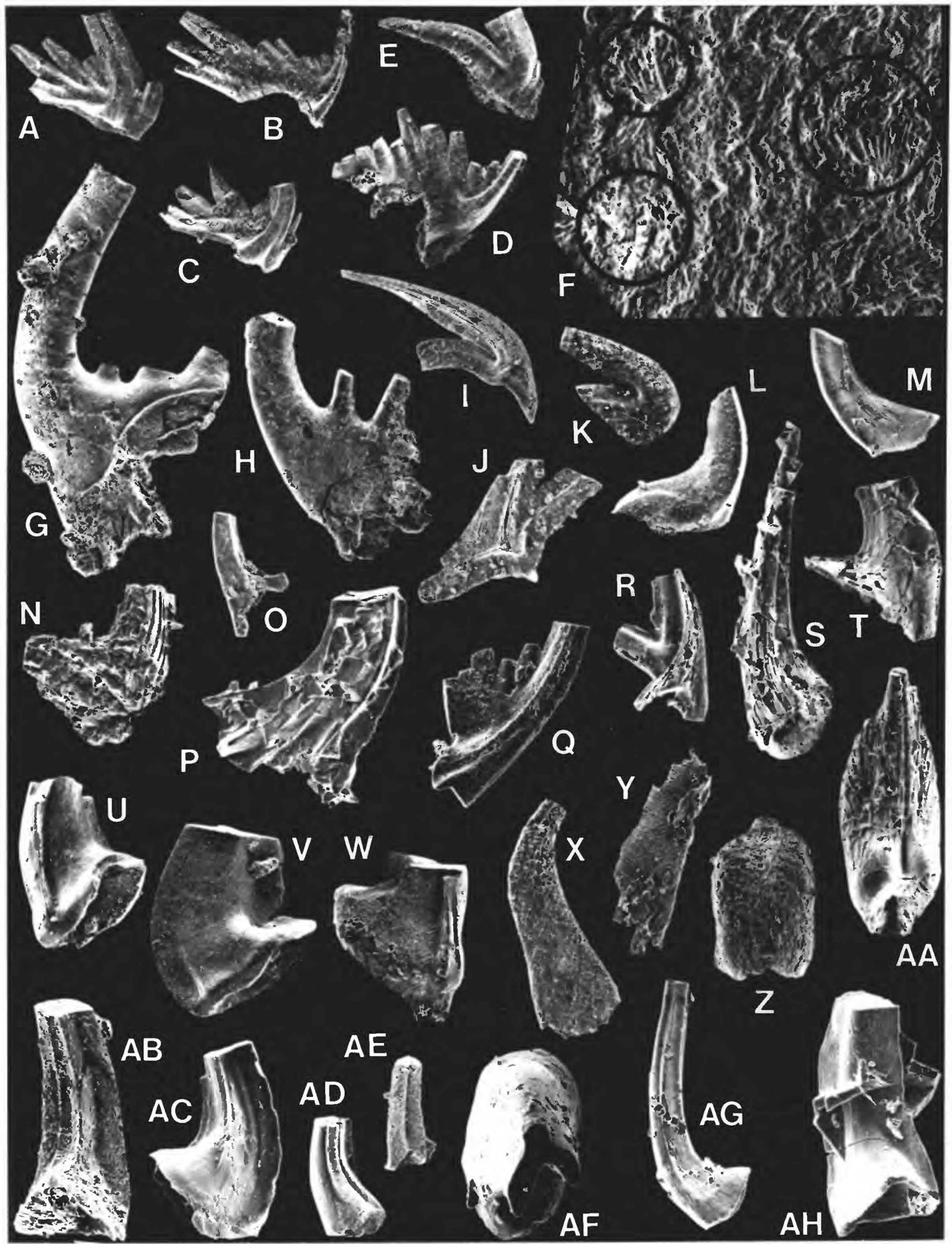




\section{PLATE III}

Middle Ordovician conodonts from northwestern New Jersey and immediately adjacent New York

[All specimens from Jacksonburg Limestone or correlative Balmville Limestone (nearb"' in New York), except figs. $\mathrm{H}, \mathrm{AA}$, and AB.]

A-D. Icriodella superba Rhodes. $\mathrm{Pa}$ (lateral and upper views), $\mathrm{Sa}$ (posterolateral view), and $\mathrm{Pb}$ elements, respectively. Loc. PI-1, USGS 11251-CO, USNM 486982-85.

E. Polyplacognathus ramosus Stauffer. Upper view of Pa element. Lower part of Brlmville Limestone, at loc. 3B of Savoy, (1981), at approx. $4 \mathrm{mi}$. E of SE corner of Unionville quadrangle, in adjacent New York, USGS 9386-CO, USNM 486986.

F-H. Amorphognathus superbus (Rhodes). $\mathrm{Pb}$ (holodontiform) elements. $\mathrm{F}$ and $\mathrm{G}$ from Jacksonburg Limestone at same loc. as figs. A-D (= loc. 6 of Barnett, 1965); H, fcr comparison, from Upper Ordovician Kope Formation, northern Kentucky, USGS 8538-CO, USNM 486987-89.

I. Paroistodus? venustus (Stauffer). Oistodontiform element, from basal meter of Balmville Limestone at loc. 2 of Savoy (1981), Goshen quadrangle, NY, USGS 9390-CO, Ir120, USNM 486990.

J, K. Ramiform elements (Sd and Sa) of either Amorphognathus superbus or Rhodesognathus elegans. Jacksonburg Limestone, same loc. as figs. A-D, USNM 486991, 92.

L. Rhodesognathus elegans (Rhodes). P element. Same loc. as figs. A-D, USNM 486993.

$\mathrm{M}-\mathrm{Q}$. Aphelognathus politus (Hinde). $\mathrm{Pa}$, and posterolateral views of $\mathrm{Sb}, \mathrm{Pb}, \mathrm{M}$, and $\mathrm{Sa}$ elements, respectively; figs. M, P, Q from Jacksonburg Limestone above basal cobblebearing beds at loc. BLR-1; figs. $\mathrm{N}$ and $\mathrm{O}$ from lower part of Balmville Limestone at same loc. as fig. E (loc. 3B of Savoy, 1981), USGS 9384-CO and 9385-CO, USNM 486994, 98.

R, S. Plectodina tenuis (Branson \& Mehl). Pa and posterolateral view of Sb element. Same loc. as figs. A-D, USNM 486999, 487000.

T, U. Plectodina? sp. $\mathrm{Pb}(\mathrm{X} 120)$ and $\mathrm{Pa}$ elements; lower part of Balmville Limestone, same loc. as fig. E, USNM 487001, 02.

V-Z. Phragmodus undatus Branson \& Mehl. P (figs. V-X) and S (figs. Y, Z) elements from deformed $(\mathrm{V}, \mathrm{X}, \mathrm{Z})$ and non-deformed $(\mathrm{W}, \mathrm{Y})$ strata. Elements $\mathrm{V}, \mathrm{X}, \mathrm{Z}$ from loc. BL-1, USGS 9382-CO; $W$ and $Y$ from loc. BLR-1; USNM 487003-07.

AA. Pygodus sp. Stereo-pair showing broken $\mathrm{Pa}$ element, partially exposed on bedding surface of fine-grained sandstone from Jutland klippe sequence, loc. HB-6, USGS 11253-CO, USNM 487008.

AB. Periodon aculeatus Hadding. Two ramiform (S) elements on bedding surface of siltstone from Jutland klippe, loc. HB-6, USGS 11250-CO, X60, USNM 487009.

AC. Drepanoistodus suberectus (Branson \& Mehl). Drepanodontiform element; same loc. as fig. E, X120, USNM 487010.

AD. Pseudooneotodus mitratus (Moskalenko). Upper view of this cap-shaped element. Same loc. as figs. A-D, USNM 487011. 
Harris, Repetski, Stamm, and Weary Plate III
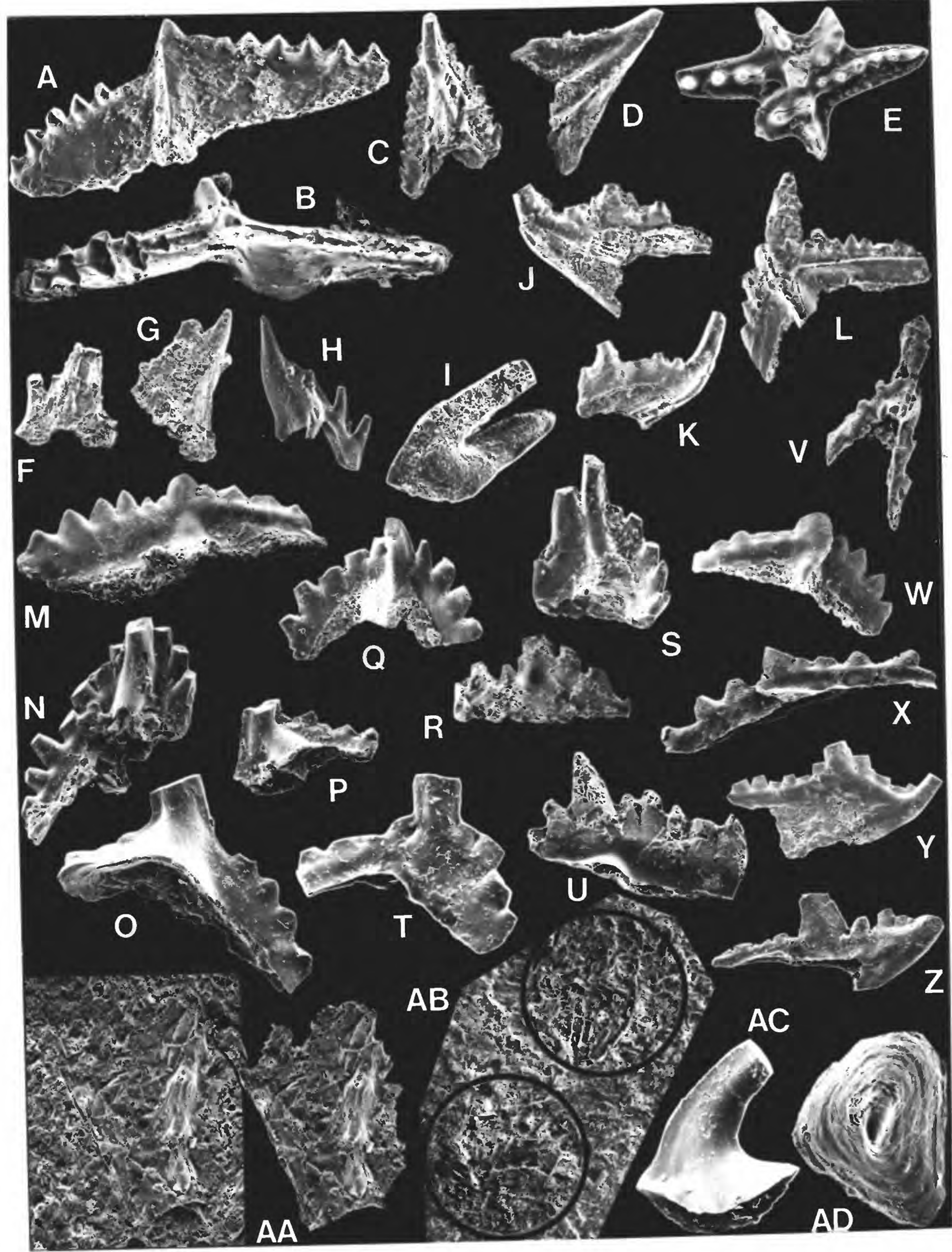


\section{PLATE IV}

Early Devonian conodonts from northwesternmost New Jersey

A-I. Ozarkodina remscheidensis (Ziegler), the most abundant species in collections from Upper Silurian rocks and in most collections from lowermost Devonian rocks in New Jersey.

A, C, D. From $1 \mathrm{~m}$ below top of Depue Limestone Member of the Coeymans

Formation, loc. CG-2, USGS colln. 12471-SD. A, C, Pa elements, inner lateral ard upper views, USNM 486876, 78. D, Pb element, outer lateral view, USNM 486879. B. Pa element, inner lateral view, $2 \mathrm{~m}$ below top of Depue Limestone Member of the Coeymans Formation, loc. FL-1, USGS colln. 12473-SD, USNM 486877. $\mathrm{E}, \mathrm{H}$. M and $\mathrm{Sb}_{2}$ elements, inner lateral views, $4 \mathrm{~m}$ below top of Thacher Member of the Manlius Limestone, loc. PJS-1, USGS colln. 12476-SD, USNM 486880, 83. F. Sa element, posterior view, $0.3 \mathrm{~m}$ above base of Shawnee Island Member of the Coeymans Formation, loc. CG-1, USGS colln. 12472-SD, USNM 486881. $\mathrm{G}, \mathrm{I}$. Sb1 and Sc elements, inner lateral views, $3 \mathrm{~m}$ below top of Thacher Member of Manlius Limestone, loc. MI-1, USNM 486882, 84.

J, P. From Stormville Member of the Coeymans Formation, loc. LM-1.

J, Pseudooneotodus beckmanni (Bischoff \& Sannemann), upper view, $0.3 \mathrm{~m}$ above base of member, USGS colln. 12474-SD, USNM 486885. P, Belodella devonica (Stauffer), $S^{\text {" }}$ element, outer lateral view, $0.6 \mathrm{~m}$ above base of member, USGS colln. 12475-SD, USNM 486886.

K-O. Oulodus cristagalli (Ziegler) from the Coeymans Formation. This species first appears in the very latest Pridolian and is an auxiliary guide to the base of the Devonian.

$\mathrm{K}, \mathrm{M}-\mathrm{O}$. $\mathrm{Pb} 1$ (lateral view), $\mathrm{Sa}$ (posterior view), $\mathrm{Sb}$, and $\mathrm{Sc}$ (lateral views) elements, 0.6 $\mathrm{m}$ above base of Ravena Member, loc. PJS-1, USNM 486887-90. $\mathrm{L}, \mathrm{Pb} 2$, lateral view, $8 \mathrm{~m}$ above base of Shawnee Island Member, loc. MI-1, USNM 486891.

Q-V. Icriodus woschmidti Ziegler from the Coeymans Formation. This species is a guide to the base of the Devonian.

$\mathrm{Q}, \mathrm{R}, \mathrm{U} . \mathrm{Pa}$ (upper view), $\mathrm{Pb}$ (postero-lateral view), and an adenticulate $\mathrm{Sc}$ element (inner lateral view), Shawnee Island Member ( $Q$ and $U, 1 \mathrm{~m}$ above base, USGS colln. 12477-SD; R, 0.6 m above base), loc. MI-1, USNM 486892-94. S. M element, inner lateral view, same collection as P, USNM 486895. $\mathrm{T}, \mathrm{V}$. Adenticulate and denticulate Sb elements, inner and outer lateral views, same collection as F, USNM 486896, 97. 
Harris, Repetski, Stamm, and Weary Plate IV

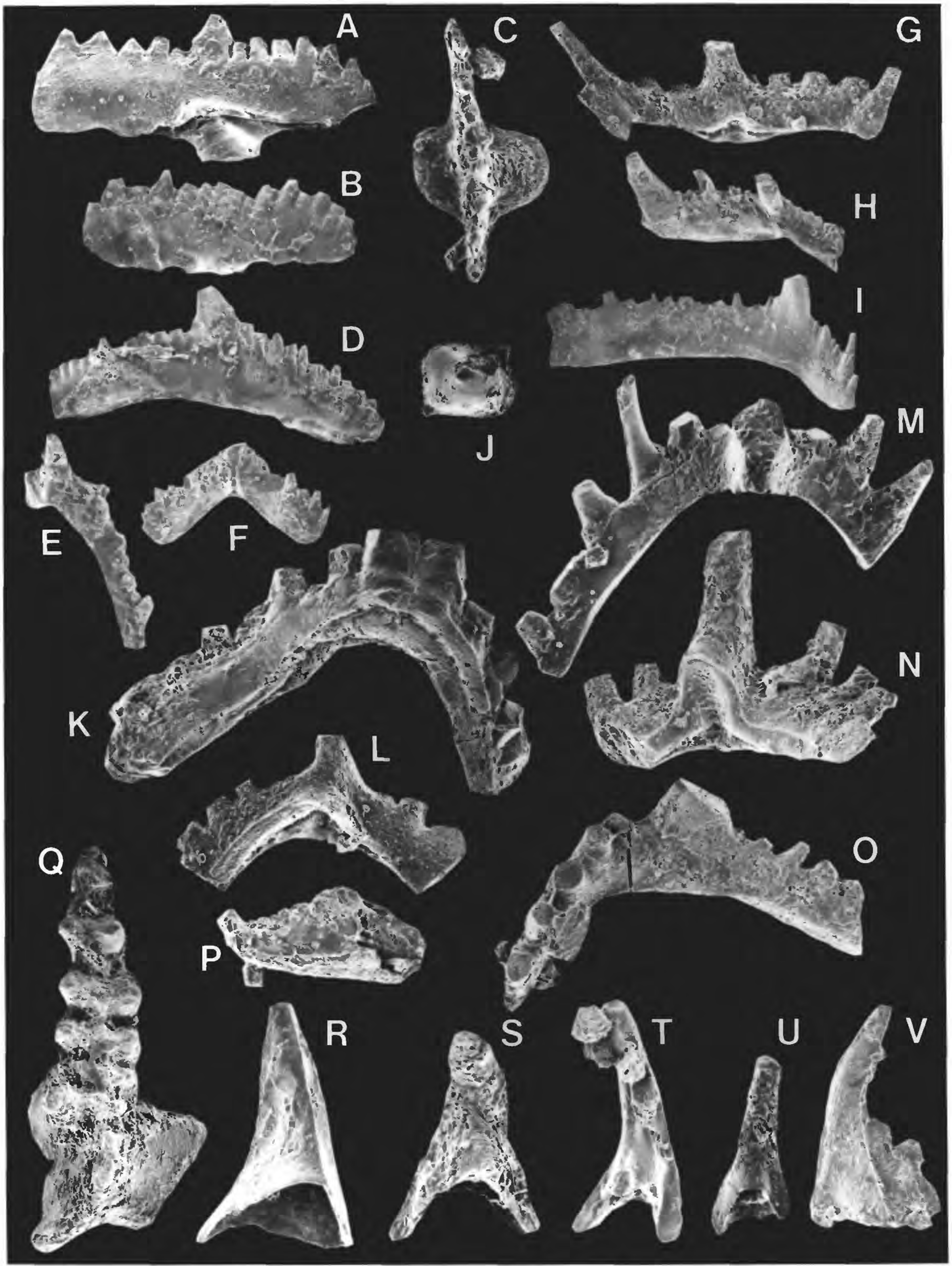

